ORNL/TM-11909

Energy Systems Environmental Restoration Program ORNL Environmental Restoration Program

Data Base Dictionary for the Oak Ridge Reservation Hydrology and Geology Study Groundwater Data Base

B. K Thompson

Date Issued-April 1993

Prepared by

Environmental Sciences Division

Oak Ridge National Laboratory

ESD Publication $\mathbf{4 0 5 0}$

Prepared for

U.S. Department of Energy

Office of Environmental Restoration and Waste Management under budget and reporting code EW 20

OAK RIDGE NATIONAL LABORATORY

Oak Ridge, Tennessee 37831-6285

managed by

MARTIN MARIETTA ENERGY SYSTEMS, INC.

for the

U.S. DEPARTMENT OF ENERGY

under contract DE-AC05-84OR21400 


\section{Author Affiliation}

B. K. Thompson is a research associate in the Department of Civil Engineering at The University of Tennessee, Knoxville. 


\section{CONTENTS}

FIGURES $\ldots \ldots \ldots \ldots \ldots \ldots \ldots \ldots \ldots \ldots \ldots \ldots \ldots \ldots \ldots \ldots \ldots \ldots, \mathbf{v}$

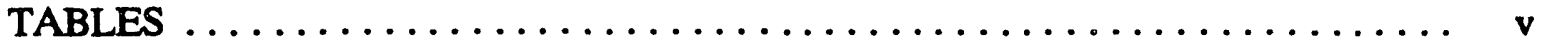

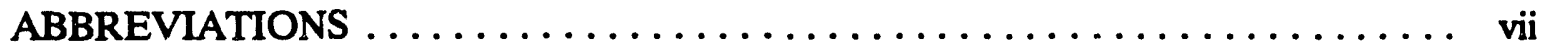

EXECUTIVE SUMMARY $\ldots \ldots \ldots \ldots \ldots \ldots \ldots \ldots \ldots \ldots \ldots$, ix

1. INTRODUCTION $\ldots \ldots \ldots \ldots \ldots \ldots \ldots \ldots \ldots \ldots \ldots \ldots \ldots \ldots \ldots \ldots \ldots$

2. STRUCTURE OF THE DATA BASE $\ldots \ldots \ldots \ldots \ldots \ldots \ldots \ldots \ldots \ldots, 2$

3. CONVENTIONS OF THE DATA BASE $\ldots \ldots \ldots \ldots \ldots \ldots \ldots \ldots \ldots, 5$

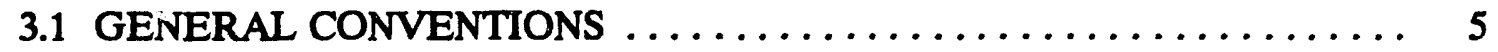

3.2 FIELD AND MISCELLANEOUS FILE CONVENTIONS $\ldots \ldots \ldots \ldots \ldots 5$

3.3 WELL CONVENTIONS $\ldots \ldots \ldots \ldots \ldots \ldots \ldots \ldots \ldots \ldots \ldots \ldots$

4. USE OF THE DATA BASE $\ldots \ldots \ldots \ldots \ldots \ldots \ldots \ldots \ldots \ldots \ldots, 8$

5. FILE DESCRIPTIONS $\ldots \ldots \ldots \ldots \ldots \ldots \ldots \ldots \ldots \ldots \ldots \ldots \ldots \ldots \ldots \ldots$

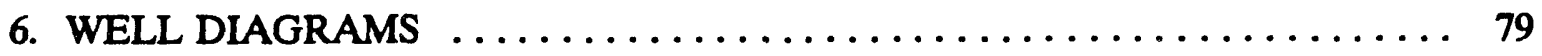

7. CROSS-REFERENCE OF PARAMETERS, VARIABLES, AND DATA BASE FILES $\ldots \ldots \ldots \ldots \ldots \ldots \ldots \ldots \ldots \ldots \ldots, 87$

8. REFERENCES $\ldots \ldots \ldots \ldots \ldots \ldots \ldots \ldots \ldots \ldots \ldots \ldots \ldots \ldots \ldots \ldots \ldots \ldots \ldots$ 


\section{FIGURES}

6.1 Diagram of an open well with one casing $\ldots \ldots \ldots \ldots \ldots \ldots$

6.2 Diagram of an open well with two casings $\ldots \ldots \ldots \ldots \ldots \ldots \ldots \ldots \ldots$

6.3 Diagram of an open well with three casings $\ldots \ldots \ldots \ldots \ldots \ldots \ldots \ldots \ldots$

6.4 Diagram of a screened well with one casing $\ldots \ldots \ldots \ldots \ldots \ldots \ldots \ldots \ldots$

6.5 Diagram of a screened well with two casings $\ldots \ldots \ldots \ldots \ldots \ldots \ldots \ldots$

6.6 Diagram of a screened well with three casings $\ldots \ldots \ldots \ldots \ldots \ldots \ldots$

\section{TABLES}

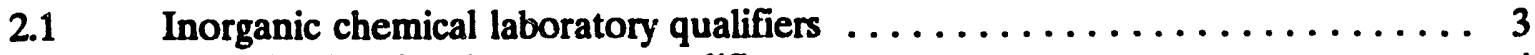

2.2 Organic chemical laboratory qualifiers $\ldots \ldots \ldots \ldots \ldots \ldots \ldots \ldots \ldots \ldots \ldots$

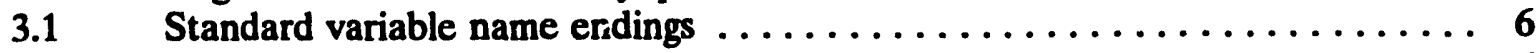

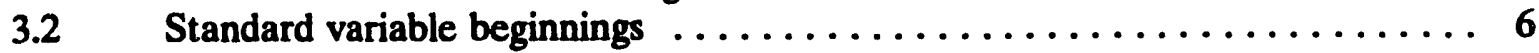

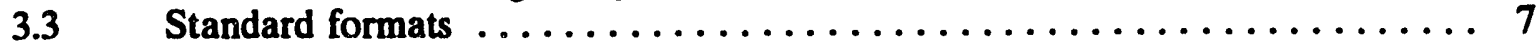

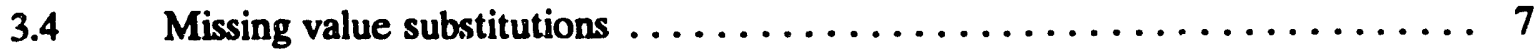

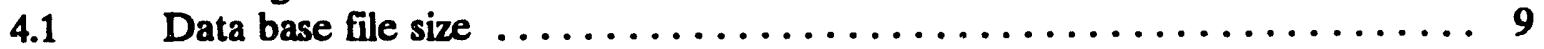

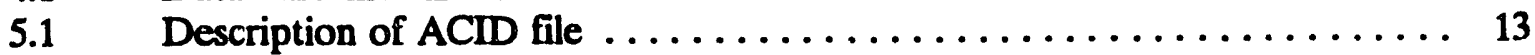

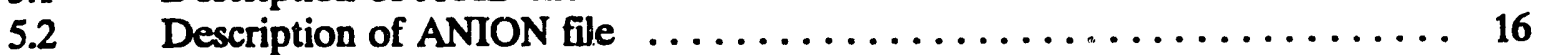

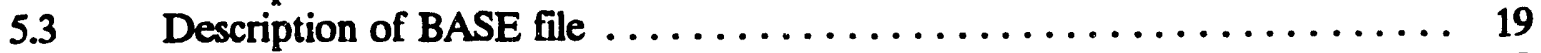

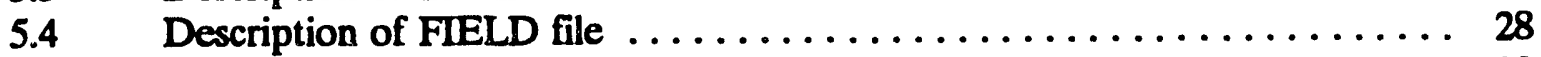

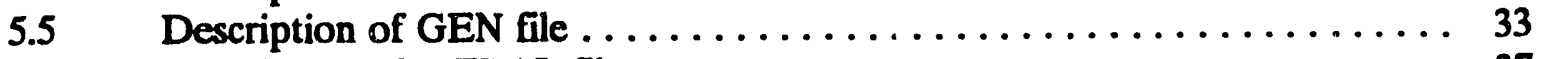

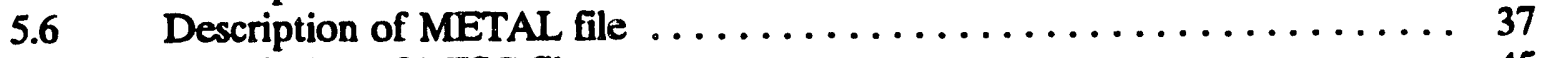

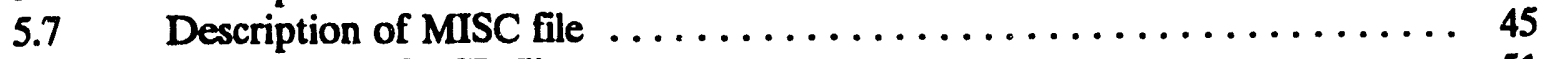

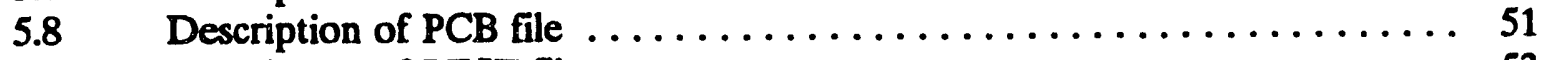

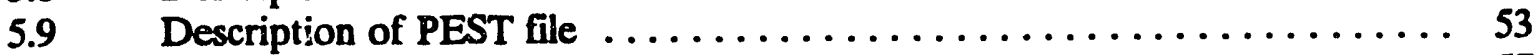

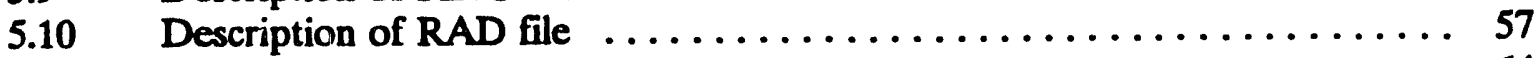

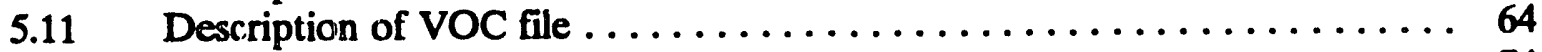

5.12 Description of WCON file $\ldots \ldots \ldots \ldots \ldots \ldots \ldots \ldots \ldots \ldots \ldots \ldots \ldots$

7.1 Cross-reference of parameters, variables, and data base files . . . . . . . 89 


\section{ABBREVIATIONS}

$\begin{array}{ll}\text { AA } & \text { atomic absorption } \\ \text { AAS } & \text { atomic absorption spectroscopy } \\ \text { CVAA } & \text { cold vapor atomic absorption } \\ \text { GC/MS } & \text { gas chromatograph/mass spectroscopy } \\ \text { ICAP } & \text { inductively coupled arc plasma } \\ \text { MSA } & \text { method of standard additions } \\ \text { NTU } & \text { normal turbidity units } \\ \text { ORNL } & \text { Oak Ridge National Laboratory } \\ \text { ORR } & \text { Oak Ridge Reservation } \\ \text { ORRHAGS } & \text { Oak Ridge Reservation Hydrology and Geology Study } \\ \text { TIC } & \text { tentatively identified compound }\end{array}$




\section{EXECUTIVE SUMMARY}

The Oak Ridge Reservation Hydrology and Geology Study (ORRHAGS) Groundwater Data Base has been compiled to consolidate groundwater data from the three U.S. Department of Energy facilities located on the Oak Ridge Reservation: the Oak Ridge K-25 Site, the Oak Ridge National Laboratory, and the Oak Ridge Y-12 Plant. Each of these facilities maintains its own groundwater and well construction data bases. Data were extracted fröm the existing data bases, converted to a consistent format, and integrated into the ORRHAGS Groundwater Data Base structures. This data base dictionary describes the data contained in the ORRHAGS Groundwater Data Base and contains information on data base structure, conventions, contents, and use. 


\section{INTRODUCTION}

The Oak Ridge Reservation Hydrology and Geology Stady (ORRHAGS) Groundwater Data Base has been compiled to consolidate groundwater data from the three U.S. Department of Energy facilities located on the Oak Ridge Reservation (ORR): the Oak Ridge K-25 Site, the Oak Ridge National Laboratory (ORNL), and the Oak Ridge Y-12 Plant. Each of these facilities maintains its own groundwater and well construction data bases. Data were extracted from the existing data bases, converted to a consistent format, and integrated into the ORRHAGS Groundwater Data Base structures. The ORRHAGS Groundwater Data Base, now in its first version, includes results of groundwater chemical analyses, field parameters measured during sample collection, water level measurements, information on hydrogeologic parameters, general well information, and well construction data for $\sim 2500$ wells throughout the ORR. Typically, the data coverage for a specific well is not complete, and only some of the above information is available.

The data base is not intended for regulatory compliance or administrative applications. To facilitate use of the ORRHAGS data base, this data base dictionary has been prepared to describe the data contained in the ORRHAGS Groundwater Data Base. The dictionary contains information on data base structure, conventions, contents, and use. Objectives for the data base; roles and responsibilities of the personnel involved; and flow, updates, and storage of the data are documented in the management plan for the ORRHAGS Groundwater Data Base (Thompson 1993).

The K-25 Site data extracted for the first version of the ORRHAGS Groundwater Data Base include groundwater information dating from 1987 to 1989 . The data were compiled by the K-25 Site Groundwater Protection Program. They also include well construction information dating from 1986 to 1989 that was compiled by Geraghty and Miller, Inc. (1989). Any external reference, publication, or release of the K-25 Site data must be cleared by the K-25 Site Groundwater Protection Program Manager's Office, which is part of the K-25 Site Environmental Management Department.

The ORNL data extracted for the first version of the ORRHAGS Groundwater Data Base include groundwater information dating from 1985 to 1989 and well construction information dating from 1949 to 1990 . All ORNL data were compiled by the ORNL Environmental Restoration Program. Any external reference, publication, or release of ORNL groundwater data must be cleared by the ORNL Groundwater Protection Program Manager's Office, which is part of the ORNL Environmental Restoration Program.

The Y-12 Plant data extracted for the first version of the ORRHAGS Groundwater Data Base include groundwater information dating from 1986 to 1989 compiled by the Y-12 Plant Environmental Management Department and well construction information dating from 1943 to 1991 (King et al. 1991). Any external reference, release, or publication of Y-12 Plant data must be cleared by the Y-12 Plant Groundwater Protection Program Manager's Office, which is managed by the Y-12 Plant Environmental Management Department. 


\section{STRUCTURE OF THE DATA BASE}

The ORRHAGS Groundwater Data Base is stored in dBASE IV;* however, the data base is structured to be compatible with SAS† for both mainframe computers and DOSbased personal computers (PCs) and with Macintosh-compatible FoxBASE+/Mac products. The data base consists of 12 data base files: acid extractables (ACID); anions (ANION); base/neutral extractables (BASE); field parameters (FIELD); general well data (GEN); metals (METAL) - that is, cations; miscellaneous parameters (MISC); polychlorinated biphenyls (PCB); pesticides (PEST); radionuclides (RAD); volatile organic compounds (VOC); and well construction data (WCON). Section 5 describes the structure of each data base file, including the variable name, the type of variable (character, date, or numeric), the width of each variable, the number of decimal places for numeric variables, and a complete description of each variable, including units and abbreviations.

Relational data base files contain variables, called key variables, which allow the records to be identified uniquely. The key variables also make it possible to merge information from different data sets to form secondary files to meet specialized needs. The maximum number of variables that a secondary dBASE IV file can hold is 256. Within the ORRHAGS data base there are two groups of data base files; one group consists of data gathered from groundwater samples, and the other consists of data gathered from the installation of wells. Several key variables are required to uniquely identify groundwater sample data because the files contain multiple recurds for each well. The key variables for the groundwater sample files (ACID, ANION, BASE, FIELD, METAL, MISC, PCB, PEST, RAD, and VOC) are (1) the facility that installed the well (PLANT), (2) the well name (WELL), (3) the date a groundwater sample was collected (DATE_SAM), (4) whether or not it was a filtered sample (FILTER_Q), and (5) whether or not it was a duplicate sample (DUP_Q). The key variables for the well information files (GEN and WCON) are (1) PLANT and (2) WELL. The PLANT key variable is necessary because the well names are not unique throughout the ORR but only within each of the three sites. Wells are sometimes given alternate, temporary, or unofficial well names. Alias well names are stored in the GEN file (see Table 5.5, variable WELALT_1).

The groundwater sample files have laboratory qualifiers (EPA 1989) associated with the analytical results. Laboratory qualifiers are stored in an associated variable. This associated variable has the same variable name as the measured parameter with an underscore and the letter $C(C)$ at the end of the variable name. The definitions of the laboratory qualifiers are different for inorganic (Table 2.1) and organic (Table 2.2) chemicals. Inorganic chemicals are found in the ANION, METAL, and RAD files. Organic chemicals are found in the ACID, BASE, PCB, PEST, and VOC files.

*dBASE IV is the registered trademark of Ashton-Tate Corporation, Torrance, California.

†SAS is the registered trademark of SAS Institute, Inc., Cary, North Carolina.

¥FoxBASE+/Mac is the registered trademark of Fox Holdings, Inc., Perrysburg, Ohio. 
Results for radionuclides are stored in the RAD file. Counting errors, which are reported along with RAD data, are stored in an associated variable. This associated variable has the same variable name as the measured parameter with an underscore and the letter $E(E)$ at the end of the variable name.

Table 21. Inorganic chemical laboratory qualifiers

\begin{tabular}{|c|c|}
\hline Qualifier & Definition \\
\hline B & $\begin{array}{l}\text { Reported value is less than the contracted required detection } \\
\text { limit but greater than the instrument detection limit }\end{array}$ \\
\hline $\mathrm{U}$ or $<$ & Compound was analyzed for but not detected \\
\hline $\mathbf{E}$ & Value is estimated due to matrix interferences \\
\hline $\mathbf{M}$ & Duplicate injection precision criteria not met \\
\hline $\mathbf{N}$ & Spiked sample recovery not within control limits \\
\hline $\mathbf{S}$ & $\begin{array}{l}\text { Reported value was determined by the Method of Standard } \\
\text { Additions (MSA) }\end{array}$ \\
\hline W & $\begin{array}{l}\text { Postdigestion spike for furnace atomic absorption (AA) analysis } \\
\text { is out of control limits, while sample absorbance is }<50 \% \text { of } \\
\text { spike absorbance }\end{array}$ \\
\hline * & Duplicate analysis was not within control limits \\
\hline+ & Correlation coefficient for MSA was $<0.995$ \\
\hline$>$ & Beyond instrument scale \\
\hline
\end{tabular}

Source: EPA (1989). 
Table 22 Organic chemical laboratory qualifiers

\begin{tabular}{|c|c|}
\hline Qualifier & Definition \\
\hline $\mathbf{U}$ or $<$ & Compound was analyzed for but not detected \\
\hline $\mathbf{J}$ & $\begin{array}{l}\text { Value is estimated, either for a tentatively } \\
\text { identified compound (TIC) or when a compound is } \\
\text { present (spectral identification criteria are met, but } \\
\text { the value is less than the contract required } \\
\text { detection limit) }\end{array}$ \\
\hline $\mathrm{C}$ & $\begin{array}{l}\text { Pesticide results were confirmed by gas } \\
\text { chromatograph/mass spectrometer (GC/MS) }\end{array}$ \\
\hline B & $\begin{array}{l}\text { Analyte found in associated blank as well as in } \\
\text { sample }\end{array}$ \\
\hline $\mathbf{E}$ & $\begin{array}{l}\text { Concentration exceeds calibration range of GCMMS } \\
\text { instrument }\end{array}$ \\
\hline $\mathbf{D}$ & $\begin{array}{l}\text { Compound identified in an analysis at a secondary } \\
\text { dilution factor }\end{array}$ \\
\hline $\mathbf{A}$ & The TIC is a suspected aldol-condensation product \\
\hline $\mathbf{X}$ & Additional flags defined separately \\
\hline$>$ & Beyond instrument scale \\
\hline
\end{tabular}

Source: EPA (1989). 


\section{CONVENTIONS OF THE DATA BASE}

\subsection{GENERAL CONVENTIONS}

The ORRHAGS Groundwater Data Base conventions include standard variable name endings (Table 3.1), standard variable name beginnings (Table 3.2), standard formats (Table 3.3), and standard missing value substitutions (Table 3.4). Standard variable name endings are an underscore followed by a letter and represent laboratory qualifiers and special comment variables, counting error variables, logical variables (true or false), analysis method variables for metals, and well casing variables. Standard variable name beginnings are a group of characters followed by an underscore and represent date variables and source variables. Standard missing variables include missing numeric values, missing date values, and dates with the year only. The dBASE IV program does not allow numeric or date variables to be left blank.

Missing numeric entries would be replaced with the number zero. Since it is possible that zero could be an actual measured value, true missing numeric values are represented by 9999999.0000. The dBASE IV program has only one date format: a two-digit month, a twodigit day, and a two-digit year. Partial or completely missing date information is represented by standard date substitutions.

Variable names are unique in the ORRHAGS data base, except for the key variables (PLANT, WELL, DATA_SAM, DUP_Q, and FILTER_Q). Details about key variables can be found in Section 2.

\subsection{FIELD AND MISCELLANEOUS FILE CONVENTIONS}

Parameters that were measured in the field and the laboratory or parameters that were measured several times for each sample have similar variable names. Specific conductance and $\mathrm{pH}$ are measured in both the field and the laboratory. The variable names for specific conductance and $\mathrm{pH}$ are similar in the field and miscellaneous data files. These field variable names contain an underscore $F(F)$, whereas the miscellaneous variable names contain an underscore $L(L)$. Parameters that were measured multiple times per sample include specific conductance, dissolved oxygen, $\mathrm{pH}$, redox, temperature, total organic carbon, total organic chloride, and total organic halides. Multiple measurements have the same variable name with different ending numbers.

\subsection{WELL CONVENTIONS}

The wells installed on the ORR have as many as three casings. The well construction file stores information related to all of the well casings. Variables related to the innermost casing end with an underscore one (1). Variables related to the outer casing for wells with two casings and the middle casing for wells with three casings end with an underscore two (2). Variables related to the outer casing for wells with three casings end with an underscore three (3). Section 6 shows diagrams of the well construction variables for open and screened wells with one, two, and three casings. 
Table 3.1. Standard variable name endings

\begin{tabular}{|c|c|}
\hline Endings & Description \\
\hline A & $\begin{array}{l}\text { Metal samples analyzed by the atomic absorption } \\
\text { spectroscopy (AAS) method }\end{array}$ \\
\hline $\mathrm{C}$ & Laboratory or validation codes \\
\hline$-\mathbf{E}$ & Counting errors for radiochemical analyses \\
\hline $\mathbf{F}$ & Field observation \\
\hline$-\mathbf{H}$ & Metal samples analyzed by the flame-emission method \\
\hline II & $\begin{array}{l}\text { Metal samples analyzed by the inductively coupled arc } \\
\text { plasma (ICAP) method }\end{array}$ \\
\hline L & Laboratory observation \\
\hline$-\mathbf{P}$ & Metal samples analyzed by the polar method \\
\hline$-Q$ & Logical fields; $T$ represents true and $F$ represents false \\
\hline$-\mathbf{V}$ & $\begin{array}{l}\text { Metal samples analyzed by the cold vapor atomic } \\
\text { absorption (CVAA) method }\end{array}$ \\
\hline-1 & Inner well casing \\
\hline-2 & $\begin{array}{l}\text { Outer well casing for wells with two well casings or } \\
\text { middle well casing for wells with three well casings }\end{array}$ \\
\hline 3 & Outer well casing for wells with three well casings \\
\hline
\end{tabular}

Table 32 Standard variable beginnings

\begin{tabular}{ll}
\hline Beginnings & \multicolumn{1}{c}{ Variable contents } \\
\hline DATE_ & Date values \\
SRC_ & Data source \\
\hline
\end{tabular}


Table 3.3. Standard formats

\begin{tabular}{ll}
\hline Variable type & \multicolumn{1}{c}{ Format } \\
\hline Character & Capital letters \\
Date & $\begin{array}{l}\text { Two-digit month, two-digit day, two-digit } \\
\text { year: MM/DD/YY }\end{array}$ \\
Numeric & $\begin{array}{l}\text { Maximum of 16 places with } 4 \text { decimal } \\
\text { places (Numbers too large or too small } \\
\text { are represented in exponential notation.) }\end{array}$ \\
\hline
\end{tabular}

Table 3.4. Missing value substitutions

\begin{tabular}{ll}
\hline \multicolumn{1}{c}{ Missing values } & \multicolumn{1}{c}{ Substitution } \\
\hline Numeric & 9999999.0000 \\
Date & January 1, 1900: 01/01/00 \\
Missing day and month: & \\
Well construction & January 1 of the given year: 01/01/YY \\
Well destruction & December 31 of the given year: $12 / 31 / Y Y$ \\
\hline
\end{tabular}

The numbering pattern for well casings is opposite from the order of installation. Reversing the numbering ensures that information related to the central casing is always found in an _1 variable.

A few wells have two open intervals. Variables related to the innermost or deepest open interval end with an underscore one (1). The variables related to the upper open interval end with an underscore two (2). Variables related to the open interval of a well with only one open interval end with an underscore $1(1)$. A few wells contain four screened intervals. The variables related to the shallowest screened interval end with an underscore 1 (1), the variables related to the next deepest screened interval end with an underscore two (2), and so on, and the variables related to the deepest screened interval end with an underscore four (4). Variables related to the screened interval of a well with only one screened interval end with an uncierscore $1(1)$. 


\section{USE OF THE DATA BASE}

The ORRHAGS Groundwater Data Base is not the official repository of groundwater data; it was designed for research purposes only. The user should be aware that not all of the available data were incorporated into the ORRHAGS Groundwater Data Base. Data were omitted for a variety of reasons: incomplete data (i.e., missing well name), questionable data (i.e., invalid chemical name), parameters measured for a small number of observations, data inconsistent with the ORRHAGS data base structure (i.e., inability to determine if a parameter was measured in the field or in the laboratory), and data not applicable to ORRHAGS research (i.e., the time a sample was taken). Questions concerning information for a specific facility should be addressed to the Groundwater Protection Program Manager for that facility.

Extreme care should be used with the well construction data file. Available well construction data vary greatly from well to well, and only well construction data stored in the original data bases were reported. Because missing numeric values are represented by 9999999.0000, they must be removed before numeric analysis of the data can be performed. To avoid possible erroneous matches, groundwater records with missing sample dates, represented by $01 / 01 / 00$, should be removed before data sets are merged.

Section 7 is a cross-reference between the measured parameters, the variable names, and the data base file where the data are stored. Key variables and associated variables are not included in the cross-reference.

Most of the data base files are too large to fit on a 1.2-MB floppy disk (Table 4.1). Files can be transferred on a 44-MB Bernoulli disk. Requests for data from the ORRHAGS data base are currently handled on a case-by-case basis. Data release to users will generally involve a memorandum of agreement for subsequent review of planned publication or release of data acquired. Data requests should be sent to the ORRHAGS Data Base Administrator. The management plan for the ORRHAGS Groundwater Data Base (Thompson 1993) contains additional information on the transfer and manipulation of data. 
Table 4.1. Data base file size

\begin{tabular}{lr}
\hline Data base file & $\begin{array}{c}\text { Size (MB) for } \\
\text { Version 1 }\end{array}$ \\
\hline ACID_1.DBF & 0.4 \\
ANION_1.DBF & 1.1 \\
BASE_1.DBF & 2.0 \\
FIELD_1.DBF & 2.1 \\
GEN_1.DBF & 1.4 \\
METAL_1.DBF & 7.6 \\
MISC_1.DBF & 2.7 \\
PCB_1.DBF & 0.2 \\
PEST_1.DBF & 0.7 \\
RAD_1.DBF & 4.4 \\
VOC_1.DBF & 3.5 \\
WCON_1.DBF & 3.7 \\
\hline
\end{tabular}




\section{FILE DESCRIPTIONS}

\section{CONTENTS OF SECTION 5}

Table 5.1 Description of ACID file ........... 13

Table 5.2 Description of ANION file $\ldots \ldots \ldots \ldots \ldots 16$

Table 5.3 Description of BASE file . . . . . . . . 19

Table 5.4 Description of FIELD file $\ldots \ldots \ldots \ldots \ldots 28$

Table 5.5 Description of GEN file $\ldots \ldots \ldots \ldots \ldots \ldots$

Table 5.6 Description of METAL file ... . . . . 37

Table 5.7 Description of MISC file . . . . . . . 45

Table 5.8 Description of PCB file .......... 51

Table 5.9 Description of PEST file . . . . . . . 53

Table 5. 9 Description of RAD file $\ldots \ldots \ldots \ldots \ldots 57$

Table 5.11 Description of VOC file .......... 64

Table 5.12 Description of WCON file .......... 74 
Table 5.1. Description of ACID file

\begin{tabular}{|c|c|c|c|c|}
\hline $\begin{array}{l}\text { Variable } \\
\text { name }\end{array}$ & Type & Width & $\begin{array}{l}\text { Decimal } \\
\text { places }\end{array}$ & Description \\
\hline BENACD & Numeric & 16 & 4 & Concentration of benzoic acid $(\mu \mathrm{g} / \mathrm{L})$ \\
\hline BENACD_C & Character & 4 & & $\begin{array}{l}\text { Laboratory qualifiers for the } \\
\text { concentration of benzoic acid }\end{array}$ \\
\hline CLMETH & Numeric & 16 & 4 & $\begin{array}{l}\text { Concentration of 4-chloro-3- } \\
\text { methylphenol }(\mu \mathrm{g} / \mathrm{L})\end{array}$ \\
\hline CLMETH_C & Character & 4 & & $\begin{array}{l}\text { Laboratory qualifiers for the } \\
\text { concentration of 4-chloro-3-methylphenol }\end{array}$ \\
\hline CLPHEN & Numeric & 16 & 4 & Concentration of 2-chlorophenol $(\mu \mathrm{g} / \mathrm{L})$ \\
\hline CLPHEN_C & Character & 4 & & $\begin{array}{l}\text { Laboratory qualifiers for the } \\
\text { concentration of 2-chlorophenol }\end{array}$ \\
\hline DATE_SAM & Date & 8 & & $\begin{array}{l}\text { Date the groundwater sample was taken } \\
\text { (key) }\end{array}$ \\
\hline DCP_24 & Numeric & 16 & 4 & $\begin{array}{l}\text { Concentration of 2,4-dichlorophenol } \\
(\mu \mathrm{g} / \mathrm{L})\end{array}$ \\
\hline DCP_24_C & Character & 4 & & $\begin{array}{l}\text { Laboratory qualifiers for the } \\
\text { concentration of 2,4-dichlorophenol }\end{array}$ \\
\hline DMP_24 & Numeric & 16 & 4 & $\begin{array}{l}\text { Concentration of 2,4-dimethylphenol } \\
(\mu \mathrm{g} / \mathrm{L})\end{array}$ \\
\hline DMP_24_C & Character & 4 & & $\begin{array}{l}\text { Laboratory qualifiers for the } \\
\text { concentration of 2,4-dimethylphenol }\end{array}$ \\
\hline DNM_46 & Numeric & 16 & 4 & $\begin{array}{l}\text { Concentration of 4,6-dinitro-2- } \\
\text { methylphenol }(\mu \mathrm{g} / \mathrm{L})\end{array}$ \\
\hline DNM_46_C & Character & 4 & & $\begin{array}{l}\text { Laboratory qualifiers for the } \\
\text { concentration of 4,6-dinitro-2- } \\
\text { methylphenol }\end{array}$ \\
\hline DNP_24 & Numeric & 16 & 4 & $\begin{array}{l}\text { Concentration of 2,4-dinitrophenol } \\
(\mu \mathrm{g} / \mathrm{L})\end{array}$ \\
\hline
\end{tabular}


Table 5.1 (continued)

\begin{tabular}{|c|c|c|c|c|}
\hline $\begin{array}{l}\text { Variable } \\
\text { name }\end{array}$ & Type & Width & $\begin{array}{l}\text { Decimal } \\
\text { places }\end{array}$ & Description \\
\hline DNP_24_C & Character & 4 & & $\begin{array}{l}\text { Laboratory qualifiers for the } \\
\text { concentration of 2,4-dinitrophenol }\end{array}$ \\
\hline DUP_Q & Logic & 1 & & $\begin{array}{l}\text { Whether it is a duplicate sample (key) } \\
\mathrm{T}=\text { True, it is a duplicate sample } \\
\mathrm{F}=\text { False, it is not a duplicate sample }\end{array}$ \\
\hline FILTER_Q & Logic & 1 & & $\begin{array}{l}\text { Whether it is a filtered sample (key) } \\
\mathrm{T}=\text { True, it is a filtered sample } \\
\mathrm{F}=\text { False, it is not a filtered sample }\end{array}$ \\
\hline METH_2 & Numeric & 16 & 4 & Concentration of 2-methylphenol $(\mu \mathrm{g} / \mathrm{L})$ \\
\hline METH_2_C & Character & 4 & & $\begin{array}{l}\text { Laboratory qualifiers for the } \\
\text { concentration of 2-methylphenol }\end{array}$ \\
\hline METH_4 & Numeric & 16 & 4 & Concentration of 4-methylphenol $(\mu \mathrm{g} / \mathrm{L})$ \\
\hline METH_4_C & Character & 4 & & $\begin{array}{l}\text { Laboratory qualifiers for the } \\
\text { concentration of 4-methylphenol }\end{array}$ \\
\hline NITP_2 & Numeric & 16 & 4 & Concentration of 2-nitrophenol $(\mu \mathrm{g} / \mathrm{L})$ \\
\hline NITP_2_C & Character & 4 & & $\begin{array}{l}\text { Laboratory qualifiers for the } \\
\text { concentration of 2-nitrophenol }\end{array}$ \\
\hline NITP_4 & Numeric & 16 & 4 & Concentration of 4-nitrophenol $(\mu \mathrm{g} / \mathrm{L})$ \\
\hline NITP_4_C & Character & 4 & & $\begin{array}{l}\text { Laboratory qualifiers for the } \\
\text { concentration of 4-nitrophenol }\end{array}$ \\
\hline PENTCL & Numeric & 16 & 4 & $\begin{array}{l}\text { Concentration of pentachlorophenol } \\
(\mu \mathrm{g} / \mathrm{L})\end{array}$ \\
\hline PENTCL_C & Character & 4 & & $\begin{array}{l}\text { Laboratory qualifiers for the } \\
\text { concentration of pentachlorophenol }\end{array}$ \\
\hline PHENOL & Numeric & 16 & 4 & Concentration of phenol $(\mu \mathrm{g} / \mathrm{L})$ \\
\hline
\end{tabular}


Table 5.1 (continued)

Variable name Type

Decimal

PHENOL_C Character 4

PLANT

Character 3

SRC_ACID Character 30
Laboratory qualifiers for the concentration of phenol

Plant that installed the well (key)

$$
\begin{aligned}
& \mathrm{K} 25=\mathrm{K}-25 \\
& \mathrm{X} 10=\mathrm{ORNL} \\
& \mathrm{Y} 12=\mathrm{Y} \cdot 12
\end{aligned}
$$

Source of the data

The plant the data came from is listed first

$$
\begin{aligned}
& \mathrm{K} 25=\mathrm{K}-25 \\
& \mathrm{X} 10=\mathrm{ORNL} \\
& \mathrm{Y} 12=\mathrm{Y}-12
\end{aligned}
$$

The type of the original data file is listed second

CHEM = a groundwater chemical file WELL $=\mathrm{a}$ well construction file The original data file name is listed last, in parentheses

TC_245 Numeric $16 \quad 4 \quad$ Concentration of 2,4,5-trichlorophenol $(\mu \mathrm{g} / \mathrm{L})$

TC_245_C Character 4

Laboratory qualifiers for the concentration of 2,4,5-trichlorophenol

TC_246 Numeric $16 \quad 4$

Concentration of 2,4,6-trichlorophenol $(\mu \mathrm{g} / \mathrm{L})$

TC_246_C Character 4

Laboratory qualifiers for the concentration of 2,4,6-trichlorophenol

WELL Character 20
Official well name (key) 
Table 5.2. Description of ANION file

Variable name

Type Width places Description

Decimal

$\begin{array}{llll}\text { ALKBCR } & \text { Numeric } & 16 & 4\end{array}$ Concentration of bicarbonate alkalinity as $\mathrm{CaCO}_{3}(\mathrm{mg} / \mathrm{L})$

ALKBCR_C Character 4

$\begin{array}{llll}\text { ALKCAR } & \text { Numeric } & 16 & 4\end{array}$

ALKCAR_C Character 4

ALKTL

ALKTL_C Character 4

BROM

BROM_C Character 4

CHLOR Numeric 16

CHLOR_C Character 4

CYANID Numeric 16

CYANID_C Character 4

DATE SAM Date

8

DUP_Q Logical 1
Laboratory qualifiers for the concentration of bicarbonate alkalinity

Concentration of carbonate alkalinity as $\mathrm{CaCO}_{3}(\mathrm{mg} / \mathrm{L})$

Laboratory qualifiers for the concentration of carbonate alkalinity

Concentration of total alkalinity as $\cdot \mathrm{CaCO}_{3}$ (mg/L)

Laboratory qualifiers for the concentration of total alkalinity

Concentration of bromide $(\mathrm{mg} / \mathrm{L})$

Laboratory qualifiers for the concentration of bromide

Concentration of chloride $(\mathrm{mg} / \mathrm{L})$

Laboratory qualifiers for the concentration of chloride

Concentration of total cyanide $(\mathrm{mg} / \mathrm{L})$

Laboratory qualifiers for the concentration of total cyanide

Date the groundwater sample was taken (key)

Whether it is a duplicate sample (key) $\mathrm{T}=$ True, it is a duplicate sample $F=F a l s e$, it is not a duplicate sample 
Table 5.2 (continued)

\begin{tabular}{|c|c|c|c|c|}
\hline $\begin{array}{l}\text { Variable } \\
\text { name }\end{array}$ & Type & Width & $\begin{array}{l}\text { Decimal } \\
\text { places }\end{array}$ & Description \\
\hline FILTER_Q & Logical & 1 & & $\begin{array}{l}\text { Whether it is a filtered sample (key) } \\
\begin{aligned} T & =\text { True, it is a filtered sample } \\
\mathrm{F} & =\text { False, it is not a filtered sample }\end{aligned}\end{array}$ \\
\hline FLUOR & Numeric & 16 & 4 & Concentration of fluoride $(\mathrm{mg} / \mathrm{L})$ \\
\hline FLUOR_C & Character & 4 & & $\begin{array}{l}\text { Laboratory qualifiers for the } \\
\text { concentration of fluoride }\end{array}$ \\
\hline IODIDE & Numeric & 16 & 4 & Concentration of iodide $(\mathrm{mg} / \mathrm{L})$ \\
\hline IODIDE_C & Character & 4 & & $\begin{array}{l}\text { Laboratory qualifiers for the } \\
\text { concentration of iodide }\end{array}$ \\
\hline NITRAT & Numeric & 16 & 4 & Concentration of nitrate as $N(\mathrm{mg} / \mathrm{L})$ \\
\hline NITRAT_C & Character & 4 & & $\begin{array}{l}\text { Laboratory qualifiers for the } \\
\text { concentration of nitrate }\end{array}$ \\
\hline NITRIT & Numeric & 16 & 4 & Concentration of nitrite as $N(\mathrm{mg} / \mathrm{L})$ \\
\hline NITRIT_C & Character & 4 & & $\begin{array}{l}\text { Laboratory qualifiers for the } \\
\text { concentration of nitrite }\end{array}$ \\
\hline PHOSPA & Numeric & 16 & 4 & Concentration of phosphate $(\mathrm{mg} / \mathrm{L})$ \\
\hline PHOSPA_C & Character & 4 & & $\begin{array}{l}\text { Laboratory qualifiers for the } \\
\text { concentration of phosphate }\end{array}$ \\
\hline PLANT & Character & 3 & & $\begin{array}{l}\text { Plant that installed the well (key) } \\
\begin{aligned} \text { K25 } & =\mathrm{K}-25 \\
\mathrm{X} 10 & =\mathrm{ORNL} \\
\mathrm{Y} 12 & =\mathrm{Y}-12\end{aligned}\end{array}$ \\
\hline
\end{tabular}


Table 5.2 (continued)

\begin{tabular}{|c|c|c|c|c|}
\hline $\begin{array}{l}\text { Variable } \\
\text { name }\end{array}$ & Type & Width & $\begin{array}{l}\text { Decimal } \\
\text { places }\end{array}$ & Description \\
\hline SRC_AN & Character & 30 & & $\begin{array}{l}\text { Source of the data } \\
\text { The plant the data came from is listed } \\
\text { first } \\
\text { K25 = K-25 } \\
\text { X10 = ORNL } \\
\text { Y12 = Y-12 } \\
\text { The type of the original data file is listed } \\
\text { second } \\
\text { CHEM = a groundwater analyses file } \\
\text { WELL = a well construction file } \\
\text { The original data file name is listed last, } \\
\text { in parentheses }\end{array}$ \\
\hline SULFAT & Numeric & 16 & 4 & Concentration of sulfate $(\mathrm{mg} / \mathrm{L})$ \\
\hline SULFAT_C & Character & 4 & & $\begin{array}{l}\text { Laboratory qualifiers for the } \\
\text { concentration of sulfate }\end{array}$ \\
\hline SULFID & Numeric & 16 & 4 & Concentration of sulfide $(\mathrm{mg} / \mathrm{L})$ \\
\hline SULFID_C & Character & 4 & & $\begin{array}{l}\text { Laboratory qualifiers for the } \\
\text { concentration of sulfide }\end{array}$ \\
\hline WELL & Character & 20 & & Official well name (key) \\
\hline
\end{tabular}


Table 5.3. Description of BASE file

\begin{tabular}{|c|c|c|c|c|}
\hline $\begin{array}{l}\text { Variable } \\
\text { name }\end{array}$ & Type & Width & $\begin{array}{l}\text { Decimal } \\
\text { places }\end{array}$ & Description \\
\hline ACNTHE & Numeric & 16 & 4 & Concentration of acenaphthene $(\mu \mathrm{g} / \mathrm{L})$ \\
\hline ACNTHE_C & Character & 4 & & $\begin{array}{l}\text { Laboratory qualifiers for the } \\
\text { concentration of acenaphthene }\end{array}$ \\
\hline ACNTHY & Numeric & 16 & 4 & Concentration of acenaphthylene $(\mu \mathrm{g} / \mathrm{L})$ \\
\hline ACNTHY_C & Character & 4 & & $\begin{array}{l}\text { Laboratory qualifiers for the } \\
\text { concentration of acenaphthylene }\end{array}$ \\
\hline ANILIN & Numeric & 16 & 4 & Concentration of aniline $(\mu \mathrm{g} / \mathrm{L})$ \\
\hline ANILIN_C & Character & 4 & & $\begin{array}{l}\text { Laboratory qualifiers for the } \\
\text { concentration of aniline }\end{array}$ \\
\hline ANTHRA & Numeric & 16 & 4 & Concentration of anthracene $(\mu \mathrm{g} / \mathrm{L})$ \\
\hline ANTHRA_C & Character & 4 & & $\begin{array}{l}\text { Laboratory qualifiers for the } \\
\text { concentration of anthracene }\end{array}$ \\
\hline BEN & Numeric & 16 & 4 & Concentration of benzidine $(\mu \mathrm{g} / \mathrm{L})$ \\
\hline BEN_C & Character & 4 & & $\begin{array}{l}\text { Laboratory qualifiers for the } \\
\text { concentration of benzidine }\end{array}$ \\
\hline BENAAN & Numeric & 16 & 4 & $\begin{array}{l}\text { Concentration of benzo }[a] \text { anthracene } \\
(\mu \mathrm{g} / \mathrm{L})\end{array}$ \\
\hline BENAAN_C & Character & 4 & & $\begin{array}{l}\text { Laboratory qualifiers for the } \\
\text { concentration of benzo }[a] \text { anthracene }\end{array}$ \\
\hline BENBFL & Numeric & 16 & 4 & $\begin{array}{l}\text { Concentration of benzo[b] lluoranthene } \\
(\mu \mathrm{g} / \mathrm{L})\end{array}$ \\
\hline BENBFL_C & Character & 4 & & $\begin{array}{l}\text { Laboratory qualifiers for the } \\
\text { concentration of benzo[b]fluoranthene }\end{array}$ \\
\hline BENKFL & Numeric & 16 & 4 & $\begin{array}{l}\text { Concentration of benzo }[k] \text { fluoranthene } \\
(\mu \mathrm{g} / \mathrm{L})\end{array}$ \\
\hline
\end{tabular}


Table 5.3 (continued)

Variable

Decimal

name

Type

Width places

Description

BENKFL_C Character 4

$\begin{array}{llll}\text { BENPER Numeric } & 16 & 4\end{array}$

BENPER_C Character 4

$\begin{array}{llll}\text { BENPYR Numeric } & 16 & 4\end{array}$

BENPYR_C Character 4

BENZAL Numeric 16

BENZAL_C Character 4

$\begin{array}{llll}\text { BISETH } & \text { Numeric } & 16 & 4\end{array}$

BISETH_C Character 4

$\begin{array}{llll}\text { BISETP } & \text { Numeric } & 16 & 4\end{array}$

BISETP_C Character 4

$\begin{array}{llll}\text { BISMTH } & \text { Numeric } & 16 & 4\end{array}$

BISMTH_C Character 4

$\begin{array}{llll}\text { BISPHT } & \text { Numeric } & 16 & 4\end{array}$
Laboratory qualifiers for the concentration of benzo[ $k]$ fluoranthene

Concentration of benzo[ghi]perylene $(\mu \mathrm{g} / \mathrm{L})$

Laboratory qualifiers for the concentration of benzo[ghi]perylene

Concentration of benzo[a]pyrene $(\mu \mathrm{g} / \mathrm{L})$

Laboratory qualifiers for the concentration of benzo $[a]$ pyrene

Concentration of benzyl alcohol $(\mu \mathrm{g} / \mathrm{L})$

Laboratory qualifiers for the concentration of benzyl alcohol

Concentration of bis(2-chloroethyl)ether $(\mu \mathrm{g} / \mathrm{L})$

Laboratory qualifiers is the concentration of bis(2-chloroethyl)ether

Concentration of bis(2-chloroisopropyl)ether $(\mu \mathrm{g} / \mathrm{L})$

Laboratory qualifiers for the concentration of bis(2-chloroisopropyl)ether

Concentration of

bis(2-chloroethoxy)methane $(\mu \mathrm{g} / \mathrm{L})$

Laboratory qualifiers for the concentration of bis(2-chloroethoxy)methane

Concentrationofbis(2-ethylhexyl)phthalate $(\mu \mathrm{g} / \mathrm{L})$ 
Table 5.3 (continued)

\begin{tabular}{|c|c|c|c|c|}
\hline $\begin{array}{l}\text { Variable } \\
\text { name }\end{array}$ & Type & Width & $\begin{array}{l}\text { Decimal } \\
\text { places }\end{array}$ & Description \\
\hline BISPHT_C & Character & 4 & & $\begin{array}{l}\text { Laboratory qualifiers for the } \\
\text { concentration of } \\
\text { bis(2-ethylhexyl)phthalate }\end{array}$ \\
\hline BROPHE & Numeric & 16 & 4 & $\begin{array}{l}\text { Concentration of 4-bromophenyl phenyl } \\
\text { ether }(\mu \mathrm{g} / \mathrm{L})\end{array}$ \\
\hline BROPHE_C & Character & 4 & & $\begin{array}{l}\text { Laboratory qualifiers for the } \\
\text { concentration of 4-bromophenyl phenyl } \\
\text { ether }\end{array}$ \\
\hline BUTBPH & Numeric & 16 & 4 & $\begin{array}{l}\text { Concentration of butyl benzyl phthalate } \\
(\mu \mathrm{g} / \mathrm{L})\end{array}$ \\
\hline BUTBPH_C & Character & 4 & & $\begin{array}{l}\text { Laboratory qualifiers for the } \\
\text { concentration of butyl benzyl phthalate }\end{array}$ \\
\hline CHLANI & Numeric & 16 & 4 & Concentration of 4-chloroaniline $(\mu \mathrm{g} / \mathrm{L})$ \\
\hline CHLANI_C & Character & 4 & & $\begin{array}{l}\text { Laboratory qualifiers for the } \\
\text { concentration of 4-chloroaniline }\end{array}$ \\
\hline CHLNAP & Numeric & 16 & 4 & $\begin{array}{l}\text { Concentration of 2-chloronaphthalene } \\
(\mu \mathrm{g} / \mathrm{L})\end{array}$ \\
\hline CHLNAP_C & Character & 4 & & $\begin{array}{l}\text { Laboratory qualifiers for the } \\
\text { concentration of 2-chloronaphthalene }\end{array}$ \\
\hline CHLPPE & Numeric & 16 & 4 & $\begin{array}{l}\text { Concentration of 4-chlorophenyl phenyl } \\
\text { ether }(\mu \mathrm{g} / \mathrm{L})\end{array}$ \\
\hline CHLPPE_C & Character & 4 & & $\begin{array}{l}\text { Laboratory qualifiers for the } \\
\text { concentration of 4-chlorophenyl phenyl } \\
\text { ether }\end{array}$ \\
\hline CHRYSE & Numeric & 16 & 4 & Concentration of chrysene $(\mu \mathrm{g} / \mathrm{L})$ \\
\hline CHRYSE_C & Character & 4 & & $\begin{array}{l}\text { Laboratory qualifiers for the } \\
\text { concentration of chrysene }\end{array}$ \\
\hline
\end{tabular}


Table 5.3 (continued)

\begin{tabular}{|c|c|c|c|c|}
\hline $\begin{array}{l}\text { Variable } \\
\text { name }\end{array}$ & Type & Width & $\begin{array}{l}\text { Decimal } \\
\text { places }\end{array}$ & Description \\
\hline D_24 & Numeric & 16 & 4 & Concentration of 2,4-D $(\mu \mathrm{g} / \mathrm{L})$ \\
\hline D_24_C & Character & 4 & & $\begin{array}{l}\text { Laboratory qualifiers for the } \\
\text { concentration of } 2,4-\mathrm{D}\end{array}$ \\
\hline DATE_SAM & Date & 8 & & $\begin{array}{l}\text { Date the groundwater sample was taken } \\
\text { (key) }\end{array}$ \\
\hline DIACAL & Numeric & 16 & 4 & $\begin{array}{l}\text { Concentration of diacetone alcohol } \\
(\mu \mathrm{g} / \mathrm{L})\end{array}$ \\
\hline DIACAL_C & Character & 4 & & $\begin{array}{l}\text { Laboratory qualifiers for the } \\
\text { concentration of diacetone alcohol }\end{array}$ \\
\hline DIBANT & Numeric & 16 & 4 & $\begin{array}{l}\text { Concentration of dibenzo }[a, h] \text { anthracene } \\
(\mu \mathrm{g} / \mathrm{L})\end{array}$ \\
\hline DIBANT_C & Character & 4 & & $\begin{array}{l}\text { Laboratory qualifiers for the } \\
\text { concentration of dibenzo[ }[a, h] \text { anthracene }\end{array}$ \\
\hline DIBFUR & Numeric & 16 & 4 & Concentration of dibenzofuran $(\mu \mathrm{g} / \mathrm{L})$ \\
\hline DIBFUR_C & Character & 4 & & $\begin{array}{l}\text { Laboratory qualifiers for the } \\
\text { concentration of dibenzofuran }\end{array}$ \\
\hline DIC_12 & Numeric & 16 & 4 & $\begin{array}{l}\text { Concentration of 1,2-dichlorobenzene } \\
(\mu \mathrm{g} / \mathrm{L})\end{array}$ \\
\hline DIC_12_C & Character & 4 & & $\begin{array}{l}\text { Laboratory qualifiers for the } \\
\text { concentration of } 1,2 \text {-dichlorobenzene }\end{array}$ \\
\hline DIC_13 & Numeric & 16 & 4 & $\begin{array}{l}\text { Concentration of 1,3-dichlorobenzene } \\
(\mu \mathrm{g} / \mathrm{L})\end{array}$ \\
\hline DIC_13_C & Character & 4 & & $\begin{array}{l}\text { Laboratory qualifiers for the } \\
\text { concentration of } 1,3 \text {-dichlorobenzene }\end{array}$ \\
\hline DIC_14 & Numeric & 16 & 4 & $\begin{array}{l}\text { Concentration of 1,4-dichlorobenzene } \\
(\mu \mathrm{g} / \mathrm{L})\end{array}$ \\
\hline
\end{tabular}


Table 5.3 (continued)

\begin{tabular}{|c|c|c|c|c|}
\hline $\begin{array}{l}\text { Variable } \\
\text { name }\end{array}$ & Type & Width & $\begin{array}{l}\text { Decimal } \\
\text { places }\end{array}$ & Description \\
\hline DIC_14_C & Character & 4 & & $\begin{array}{l}\text { Laboratory qualifiers for the } \\
\text { concentration of } 1,4 \text {-dichlorobenzene }\end{array}$ \\
\hline DIC_33 & Numeric & 16 & 4 & $\begin{array}{l}\text { Concentration of } 3,3^{\prime} \text {-dichlorobenzidine } \\
(\mu g / L)\end{array}$ \\
\hline DIC_33_C & Character & 4 & & $\begin{array}{l}\text { Laboratory qualifiers for the } \\
\text { concentration of } 3,3^{\prime} \text {-dichlorobenzidine }\end{array}$ \\
\hline DIEPHT & Numeric & 16 & 4 & Concestration of diethyl phthalate $(\mu \mathrm{g} / \mathrm{L})$ \\
\hline DIEHHT_C & Character & 4 & & $\begin{array}{l}\text { Laboratory qualifiers for the } \\
\text { concentration of diethyl phthalate }\end{array}$ \\
\hline DMTPHT & Numeric & 16 & 4 & $\begin{array}{l}\text { Concentration of dimethyl phthalate } \\
(\mu \mathrm{g} / \mathrm{L})\end{array}$ \\
\hline DMTPHT_C & Character & 4 & & $\begin{array}{l}\text { Laboratory qualifiers for the } \\
\text { concentration of dimethyl phthalate }\end{array}$ \\
\hline DNBPHT & Numeric & 16 & 4 & $\begin{array}{l}\text { Concentration of di- } n \text {-butyl phthalate } \\
(\mu \mathrm{g} / \mathrm{L})\end{array}$ \\
\hline DNBPHT_C & Character & 4 & & $\begin{array}{l}\text { Laboratory qualifiers for the } \\
\text { concentration of di-n-butyl phthalate }\end{array}$ \\
\hline DNI_24 & Numeric & 16 & 4 & $\begin{array}{l}\text { Concentration of 2,4-dinitrotoluene } \\
(\mu \mathrm{g} / \mathrm{L})\end{array}$ \\
\hline DNI_24_C & Character & 4 & & $\begin{array}{l}\text { Laboratory qualifiers for the } \\
\text { concentration of } 2,4 \text {-dinitrotoluene }\end{array}$ \\
\hline DNI_26 & Numeric & 16 & 4 & $\begin{array}{l}\text { Concentration of 2,6-dinitrotoluene } \\
(\mu \mathrm{g} / \mathrm{L})\end{array}$ \\
\hline DNI_26_C & Character & 4 & & $\begin{array}{l}\text { Laboratory qualifiers for the } \\
\text { concentration of 2,6-dinitrotoluene }\end{array}$ \\
\hline DNOCTP & Numeric & 16 & 4 & $\begin{array}{l}\text { Concentration of di- } n \text {-octyl phthalate } \\
(\mu \mathrm{g} / \mathrm{L})\end{array}$ \\
\hline
\end{tabular}


Table 5.3 (continued)

Variable

Decimal

name

Type

Width places

Description

$\begin{array}{lll}\text { DNOCTP_C } & \text { Character } & 4 \\ \text { DUP_Q } & \text { Logical } & 1\end{array}$

FILTER_Q Logical 1

\begin{tabular}{|c|c|c|c|c|}
\hline FLUORA & Numeric & 16 & 4 & Concentration of fluoranthene $(\mu \mathrm{g} / \mathrm{L})$ \\
\hline FLUORA_C & Character & 4 & & $\begin{array}{l}\text { Laboratory qualifiers for the } \\
\text { concentration of fluoranthene }\end{array}$ \\
\hline FLUORE & Numeric & 16 & 4 & Concentration of fluorene $(\mu \mathrm{g} / \mathrm{L})$ \\
\hline FLUORE_C & Character & 4 & & $\begin{array}{l}\text { Laboratory qualifiers for the } \\
\text { concentration of fluorene }\end{array}$ \\
\hline HXCBEN & Numeric & 16 & 4 & $\begin{array}{l}\text { Concentration of hexachlorobenzene } \\
(\mu \mathrm{g} / \mathrm{L})\end{array}$ \\
\hline HXCBEN_C & Character & 4 & & $\begin{array}{l}\text { Laboratory qualifiers fo: the } \\
\text { concentration of hexachlorobenzene }\end{array}$ \\
\hline HXCBUT & Numeric & 16 & 4 & $\begin{array}{l}\text { Concentration of hexachlorobutadiene } \\
(\mu \mathrm{g} / \mathrm{L})\end{array}$ \\
\hline HXCBUT_C & Character & 4 & & $\begin{array}{l}\text { Laboratory qualifiers for the } \\
\text { concentration of hexachlorobutadiene }\end{array}$ \\
\hline HXCCYC & Numeric & 16 & 4 & $\begin{array}{l}\text { Concentration of } \\
\text { hexachlorocyclopentadiene }(\mu \mathrm{g} / \mathrm{L})\end{array}$ \\
\hline HXCCYC_C & Character & 4 & & $\begin{array}{l}\text { Laboratory qualifiers for the concentration } \\
\text { of hexachlorocyclopentadiene }\end{array}$ \\
\hline HXCETA & Numeric & 16 & 4 & Concentration of hexachloroethane $(\mu \mathrm{g} / \mathrm{L})$ \\
\hline
\end{tabular}

Laboratory qualifiers for the concentration of di-n-ortyl phthalate

Whether it is a duplicate sample (key) $\mathrm{T}=$ True, it is a duplicate sample F $=$ False, it is not a duplicate sample

Whether it is a filtered sample (key) $\mathrm{T}=$ True, it is a filtered sample $F=$ False, it is not a filtered samplst 
Table 5.3 (continued)

Variable

Decimal

name

Type

Width places

Description

\begin{tabular}{|c|c|c|c|c|}
\hline HXCETA_C & Character & 4 & & $\begin{array}{l}\text { Laboratory qualifiers for the } \\
\text { concentration of hexachloroethane }\end{array}$ \\
\hline INDPYR & Numeric & 16 & 4 & $\begin{array}{l}\text { Concentration of indeno }(1,2,3-c d) \text { pyrene } \\
(\mu \mathrm{g} / \mathrm{L})\end{array}$ \\
\hline INDPYR_C & Character & 4 & & $\begin{array}{l}\text { Laboratory qualifiers for the } \\
\text { concentration of indeno }(1,2,3-c d) \text { pyrene }\end{array}$ \\
\hline ISOPRO & Numeric & 16 & 4 & Concentration of isophorone $(\mu \mathrm{g} / \mathrm{L})$ \\
\hline ISOPRO_C & Character & 4 & & $\begin{array}{l}\text { Laboratory qualifiers for the } \\
\text { concentration of isophorone }\end{array}$ \\
\hline MTNP_2 & Numeric & 16 & 4 & $\begin{array}{l}\text { Concentration of 2-methylnaphthalene } \\
(\mu \mathrm{g} / \mathrm{L})\end{array}$ \\
\hline MTNP_2_C & Character & 4 & & $\begin{array}{l}\text { Laboratory qualifiers for the } \\
\text { concentration of } 2 \text {-methylnaphthalene }\end{array}$ \\
\hline NAPHTA & Numeric & 16 & 4 & Concentration of naphthalene $(\mu \mathrm{g} / \mathrm{L})$ \\
\hline NAPHTA_C & Character & 4 & & $\begin{array}{l}\text { Laboratory qualifiers for the } \\
\text { concentration of naphthalene }\end{array}$ \\
\hline NBUTBZ & Numeric & 16 & 4 & $\begin{array}{l}\text { Concentration of } n \text {-butyl } \\
\text { benzenesulfonamide }(\mu \mathrm{g} / \mathrm{L})\end{array}$ \\
\hline NBUTBZ_C & Character & 4 & & $\begin{array}{l}\text { Laboratory qualifiers for the concentration } \\
\text { of } n \text {-butyl benzenesulfonamide }\end{array}$ \\
\hline NTAN_2 & Numeric & 16 & 4 & Concentration of 2-nitroaniline $(\mu \mathrm{g} / \mathrm{L})$ \\
\hline NTAN_2_C & Character & 4 & & $\begin{array}{l}\text { Laboratory qualifiers for the concentration } \\
\text { of 2-nitroaniline }\end{array}$ \\
\hline NTAN_3 & Numeric & 16 & 4 & Concentration of 3-nitroaniline $(\mu \mathrm{g} / \mathrm{L})$ \\
\hline NTAN_3_C & Character & 4 & & $\begin{array}{l}\text { Laboratory qualifiers for the concentration } \\
\text { of 3-nitroaniline }\end{array}$ \\
\hline
\end{tabular}


Table 5.3 (continued)

\begin{tabular}{|c|c|c|c|c|}
\hline $\begin{array}{l}\text { Variable } \\
\text { name }\end{array}$ & Type & Width & $\begin{array}{l}\text { Decimal } \\
\text { places }\end{array}$ & Description \\
\hline NTAN_4 & Numeric & 16 & 4 & Concentration of 4-nitroaniline $(\mu \mathrm{g} / \mathrm{L})$ \\
\hline NTAN_4_C & Character & 4 & & $\begin{array}{l}\text { Laboratory qualifiers for the } \\
\text { concentration of 4-nitroaniline }\end{array}$ \\
\hline NTBENZ & Numeric & 16 & 4 & Concentration of nitrobenzene $(\mu \mathrm{g} / \mathrm{L})$ \\
\hline NTBENZ_C & Character & 4 & & $\begin{array}{l}\text { Laboratory qualifiers for the } \\
\text { concentration of nitrobenzene }\end{array}$ \\
\hline NTMETH & Numeric & 16 & 4 & $\begin{array}{l}\text { Concentration of } N \text {-nitrosodimethylamine } \\
(\mu \mathrm{g} / \mathrm{L})\end{array}$ \\
\hline NTMETH_C & Character & 4 & & $\begin{array}{l}\text { Laboratory qualifiers for the } \\
\text { concentration of } N \text {-nitrosodimethylamine }\end{array}$ \\
\hline NTNPRO & Numeric & 16 & 4 & $\begin{array}{l}\text { Concentration of } N \text {-nitrosodi-n- } \\
\text { propylamine }(\mu \mathrm{g} / L)\end{array}$ \\
\hline NTNPRO_C & Character & 4 & & $\begin{array}{l}\text { Laboratory qualifiers for the } \\
\text { concentration of } N \text {-nitrosodi-n- } \\
\text { propylamine }\end{array}$ \\
\hline NTPHEN & Numeric & 16 & 4 & $\begin{array}{l}\text { Concentration of } N \text {-nitrosodiphenylamine } \\
(\mu \mathrm{g} / \mathrm{L})\end{array}$ \\
\hline NTPHEN_C & Character & 4 & & $\begin{array}{l}\text { Laboratory qualifiers for the } \\
\text { concentration of } N \text {-nitrosodiphenylamine }\end{array}$ \\
\hline PHENAN & Numeric & 16 & 4 & Concentration of phenanthrene $(\mu \mathrm{g} / \mathrm{L})$ \\
\hline PHENAN_C & Character & 4 & & $\begin{array}{l}\text { Laboratory qualifiers for the } \\
\text { concentration of phenanthrene }\end{array}$ \\
\hline PLANT & Character & 3 & & $\begin{array}{l}\text { Plant that installed the well (key) } \\
\begin{aligned} \mathrm{K} 25 & =\mathrm{K}-25 \\
\mathrm{X} 10 & =\mathrm{ORNL} \\
\mathrm{Y} 12 & =\mathrm{Y}-12\end{aligned}\end{array}$ \\
\hline PYRENE & Numeric & 16 & 4 & Concentration of pyrene $(\mu \mathrm{g} / \mathrm{L})$ \\
\hline
\end{tabular}


Table 5.3 (continued)

\begin{tabular}{|c|c|c|c|c|}
\hline $\begin{array}{l}\text { Variable } \\
\text { name }\end{array}$ & Type & Width & $\begin{array}{l}\text { Decimal } \\
\text { places }\end{array}$ & Description \\
\hline PYRENE_C & Character & 4 & & $\begin{array}{l}\text { Laboratory qualifiers for the } \\
\text { concentration of pyrene }\end{array}$ \\
\hline SRC_BASE & Character & 30 & & $\begin{array}{l}\text { Source of the data } \\
\text { The plant the data came from is listed } \\
\text { first } \\
\text { K25 = K-25 } \\
\text { X10 = ORNL } \\
\text { Y12 = Y-12 } \\
\text { The type of the original data file is listed } \\
\text { second } \\
\text { CHEM = a groundwater analyses file } \\
\text { WELL = a well construction file } \\
\text { The original data file name is listed last, } \\
\text { in parentheses }\end{array}$ \\
\hline T_245 & Numeric & 16 & 4 & Concentration of $2,4,5-\mathrm{T}(\mu \mathrm{g} / \mathrm{L})$ \\
\hline T_245_C & Character & 4 & & $\begin{array}{l}\text { Laboratory qualifiers for the } \\
\text { concentration of } 2,4,5-T\end{array}$ \\
\hline TB_124 & Numeric & 16 & 4 & $\begin{array}{l}\text { Concentration of 1,2,4-trichlorobenzene } \\
(\mu \mathrm{g} / \mathrm{L})\end{array}$ \\
\hline TB_124_C & Character & 4 & & $\begin{array}{l}\text { Laboratory qualifiers for the } \\
\text { concentration of } 1,2,4 \text {-trichlorobenzene }\end{array}$ \\
\hline WELL & Character & 20 & & Official well name (key) \\
\hline
\end{tabular}


Table 5.4. Description of FIELD file

\begin{tabular}{|c|c|c|c|c|}
\hline $\begin{array}{l}\text { Variable } \\
\text { name }\end{array}$ & Type & Width & $\begin{array}{l}\text { Decimal } \\
\text { places }\end{array}$ & Description \\
\hline CON_F1 & Numeric & 16 & 4 & $\begin{array}{l}\text { First field specific conductance of water } \\
(\mu \mathrm{mhos} / \mathrm{cm})\end{array}$ \\
\hline CON_F1_C & Character & 4 & & $\begin{array}{l}\text { Laboratory qualifiers for the first field } \\
\text { specific conductance of water }\end{array}$ \\
\hline CON_F2 & Numeric & 16 & 4 & $\begin{array}{l}\text { Second field specific conductance of } \\
\text { water }(\mu \mathrm{mhos} / \mathrm{cm})\end{array}$ \\
\hline CON_F2_C & Character & 4 & & $\begin{array}{l}\text { Laboratory qualifiers for the second field } \\
\text { specific conductance of water }\end{array}$ \\
\hline CON_F3 & Numeric & 16 & 4 & $\begin{array}{l}\text { Third field specific conductance of water } \\
(\mu \mathrm{mhos} / \mathrm{cm})\end{array}$ \\
\hline CON_F3_C & Character & 4 & & $\begin{array}{l}\text { Laboratory qualifiers for the third field } \\
\text { specific conductance of water }\end{array}$ \\
\hline CON_F4 & Numeric & 16 & 4 & $\begin{array}{l}\text { Fourth field specific conductance of } \\
\text { water }(\mu \mathrm{mhos} / \mathrm{cm})\end{array}$ \\
\hline CON_F4_C & Character & 4 & & $\begin{array}{l}\text { Laboratory qualifiers for the fourth field } \\
\text { specific conductance of water }\end{array}$ \\
\hline CON_F5 & Numeric & 16 & 4 & $\begin{array}{l}\text { Fifth field specific conductance of water } \\
(\mu \mathrm{mhos} / \mathrm{cm})\end{array}$ \\
\hline CON_F5_C & Character & 4 & & $\begin{array}{l}\text { Laboratory qualifiers for the fifth field } \\
\text { specific conductance of water }\end{array}$ \\
\hline CON_F6 & Numeric & 16 & 4 & $\begin{array}{l}\text { Sixth field specific conductance of water } \\
(\mu \mathrm{mhos} / \mathrm{cm})\end{array}$ \\
\hline CON_F6_C & Character & 4 & & $\begin{array}{l}\text { Laboratory qualifiers for the sixth field } \\
\text { specific conductance of water }\end{array}$ \\
\hline CON_F7 & Numeric & 16 & 4 & $\begin{array}{l}\text { Seventh field specific conductance of } \\
\text { water }(\mu \mathrm{mhos} / \mathrm{cm})\end{array}$ \\
\hline
\end{tabular}


Table 5.4 (continued)

\begin{tabular}{|c|c|c|c|c|}
\hline $\begin{array}{l}\text { Variable } \\
\text { name }\end{array}$ & Type & Width & $\begin{array}{l}\text { Decimal } \\
\text { places }\end{array}$ & Description \\
\hline CON_F7_C & Character & 4 & & $\begin{array}{l}\text { Laboratory qualifiers for the seventh field } \\
\text { specific conductance of water }\end{array}$ \\
\hline DATE_SAM & Date & 8 & & $\begin{array}{l}\text { Date the groundwater sample was taken } \\
\text { (key) }\end{array}$ \\
\hline DOXY_1 & Numeric & 16 & 4 & First field dissolved oxygen (ppm) \\
\hline DOXY_1_C & Character & 4 & & $\begin{array}{l}\text { Laboratory qualifiers for the first field } \\
\text { dissolved oxygen }\end{array}$ \\
\hline DOXY_2 & Numeric & 16 & 4 & Second field dissolved oxygen (ppm) \\
\hline DOXY_2_C & Character & 4 & & $\begin{array}{l}\text { Laboratory qualifiers for the second field } \\
\text { dissolved oxygen }\end{array}$ \\
\hline DOXY_3 & Numeric & 16 & 4 & Third field dissolved oxygen (ppm) \\
\hline DOXY_3_C & Character & 4 & & $\begin{array}{l}\text { Laboratory qualifiers for the third field } \\
\text { dissolved oxygen }\end{array}$ \\
\hline DOXY_4 & Numeric & 16 & 4 & Fourth field dissolved oxygen (ppm) \\
\hline DOXY_4_C & Character & 4 & & $\begin{array}{l}\text { Laboratory qualifiers for the fourth field } \\
\text { dissolved oxygen }\end{array}$ \\
\hline DUP_Q & Logical & 1 & & $\begin{array}{l}\text { Whether it is a duplicate sample (key) } \\
\begin{array}{l}T=\text { True, it is a duplicate sample } \\
\text { F }=\text { False, it is not a duplicate sample }\end{array}\end{array}$ \\
\hline FILTER_Q & Logical & 1 & & $\begin{array}{l}\text { Whether it is a filtered sample (key) } \\
\begin{aligned} T=\text { True, it is a filtered sample } \\
F=\text { False, it is not a filtered sample }\end{aligned}\end{array}$ \\
\hline PH_F1 & Numeric & 16 & 4 & First field pH (standard units) \\
\hline PH_F1_C & Character & 4 & & $\begin{array}{l}\text { Laboratory qualifiers for the first field } \\
\text { pH }\end{array}$ \\
\hline PH_F2 & Numeric & 16 & 4 & Second field pH (standard units) \\
\hline
\end{tabular}


Table 5.4 (continued)

Variable

Decimal name

Type

Width places

Description

PH_F2_C Character 4

$\begin{array}{lll}\text { PH_F3 } & \text { Numeric } & 16 \\ \text { PH_F3_C } & \text { Character } & 4\end{array}$

PH_F4 Numeric $16 \quad 4$

PH_F4_C Character 4

PH_F5 Numeric $16 \quad 4$

PH_F5_C Character 4

PH_F6 Numeric $16 \quad 4$

PH_F6_C Character 4

PH_F7 Numeric $16 \quad 4$

PH_F7_C Character 4

PLANT Character 3
Laboratory qualifiers for the second field pH

Third field $\mathrm{pH}$ (standard units)

Laboratory qualifiers for the third field pH

Fourth field $\mathrm{pH}$ (standard units)

Laboratory qualifiers for the fourth field pH

Fifth field pH (standard units)

Laboratory qualifiers for the fifth field pH

Sixth field pH (standard units)

Laboratory qualifiers for the sixth field pH

Seventh field pH (standard units)

Laboratory qualifiers for the seventh field pH

Plant that installed the well (key)

$$
\begin{aligned}
& \text { K25 }=\mathrm{K}-25 \\
& \mathrm{X} 10=\mathrm{ORNL} \\
& \mathrm{Y} 12=\mathrm{Y}-12
\end{aligned}
$$

$\begin{array}{lllll}\text { REDOX } & \text { Numeric } & 16 & 4 & \text { Field redox (mV) }\end{array}$

REDOX_C Character 4 Laboratory qualifiers for field redox 
Table 5.4 (continued)

\begin{tabular}{|c|c|c|c|c|}
\hline $\begin{array}{l}\text { Variable } \\
\text { name }\end{array}$ & Type & Width & $\begin{array}{l}\text { Decimal } \\
\text { places }\end{array}$ & Description \\
\hline SRC_FLD & Character & 30 & & $\begin{array}{l}\text { Source of the data } \\
\text { The plant the data came from is listed } \\
\text { first } \\
\text { K25 = K-25 } \\
\text { X10 = ORNL } \\
\text { Y12 = Y-12 } \\
\text { The type of the original data file is listed } \\
\text { second } \\
\text { CHEM = a groundwater analyses file } \\
\text { WELL = a well construction file } \\
\text { The original data file name is listed last, } \\
\text { in parentheses }\end{array}$ \\
\hline TMP_F1 & Numeric & 16 & 4 & First field temperature (degrees Celsius) \\
\hline TMP_F1_C & Character & 4 & & $\begin{array}{l}\text { Laboratory qualifiers for the first field } \\
\text { temperature }\end{array}$ \\
\hline TMP_F2 & Numeric & 16 & 4 & $\begin{array}{l}\text { Second field temperature (degrees } \\
\text { Celsius) }\end{array}$ \\
\hline TMP_F2_C & Character & 4 & & $\begin{array}{l}\text { Laboratory qualifiers for the second field } \\
\text { temperature }\end{array}$ \\
\hline TMP_F3 & Numeric & 16 & 4 & Third field temperature (degrees Celsius) \\
\hline TMP_F3_C & Character & 4 & & $\begin{array}{l}\text { Laboratory qualifiers for the third field } \\
\text { temperature }\end{array}$ \\
\hline TMP_F4 & Numeric & 16 & 4 & $\begin{array}{l}\text { Fourth field temperature (degrees } \\
\text { Celsius) }\end{array}$ \\
\hline TMP_F4_C & Character & 4 & & $\begin{array}{l}\text { Laboratory qualifiers for the fourth field } \\
\text { temperature }\end{array}$ \\
\hline TMP_F5 & Numeric & 16 & 4 & Fifth field temperature (degrees Celsius) \\
\hline TMP_F5_C & Character & 4 & & $\begin{array}{l}\text { Laboratory qualifiers for the fifth field } \\
\text { temperature }\end{array}$ \\
\hline
\end{tabular}


Table 5.4 (continued)

\begin{tabular}{|c|c|c|c|c|}
\hline $\begin{array}{l}\text { Variable } \\
\text { name }\end{array}$ & Type & Width & $\begin{array}{l}\text { Decimal } \\
\text { places }\end{array}$ & Description \\
\hline TMP_F6 & Numeric & 16 & 4 & Sixth field temperature (degrees Celsius) \\
\hline TMP_F6_C & Character & 4 & & $\begin{array}{l}\text { Laboratory qualifiers for the sixth field } \\
\text { temperature }\end{array}$ \\
\hline TMP_F7 & Numeric & 16 & 4 & $\begin{array}{l}\text { Seventh field temperature (degrees } \\
\text { Celsius) }\end{array}$ \\
\hline TMP_F7_C & Character & 4 & & $\begin{array}{l}\text { Laboratory qualifiers for the seventh field } \\
\text { temperature }\end{array}$ \\
\hline WELL & Character & 20 & & Official well name (key) \\
\hline
\end{tabular}


Table 55. Description of GEN file

Variable name

Type

Decimal

Width places Description

ACT_INAC Character 10

Whether it is an active or an inactive well ACTIVE = it is an active well (i.e., the well is available for sampling) INACTTVE $=$ it is an inactive well (i.e., the well has been destroyed, capped, covered, or damaged)

UNKNOWN = the wells availability for sampling is not known

AQUIFER Character 20

The generalized aquifers that the packed interval or open interval of a well is completed

UNC = Unconsolidated overburden

BRW = Weathered bedrock

BDR = Unweathered bedrock

CHMDAT Character 120

List of the data sets that contain groundwater analysis results for each well

$$
\begin{aligned}
& \text { ACID = ACID_1.DBF } \\
& \text { ANION = ANION_1.DBF } \\
& \text { BASE = BASE_1.DBF } \\
& \text { FIELD = FIELD_1.DBF } \\
& \text { METAL = METĀ__1.DBF } \\
& \text { MISC = MISC_1.DBF } \\
& \text { PCB = PCB_1.DBF } \\
& \text { PEST = PEST_1.DBF } \\
& \text { RAD = RAD_1.DBF } \\
& \text { VOC = VOC_1.DBF }
\end{aligned}
$$

$\begin{array}{llll}\text { EAST } & \text { Numeric } & 16 & 4\end{array}$

East coordinate. Coordinate grid system is found in the GRID variable

$\begin{array}{llll}\text { ELEVAT } & \text { Numeric } & 16 & 4\end{array}$

Ground surface elevation of the well (feet above mean sea level) 
Table 5.5 (continuced)

$\begin{array}{lll}\text { Variable } & \text { Type } \quad \text { Width } \begin{array}{l}\text { Decimal } \\ \text { places }\end{array}\end{array}$

GEO_FORM Character 72

GRID

Character

15

$\begin{array}{llll}\text { HYDCON } & \text { Numeric } & 16 & 4\end{array}$

LOGS_Q Logical 1
Geologic formation that the packed interval or open interval of a well is completed. Y-12 data contain all of the formations a well penetrated and some of the depths where the formations were encountered (data separated by ). Y-12 data are prefaced with the major formation group separated from more detailed divisions by a colon

$A=$ Stockdale Unit $A$
$B=$ Stockdale Unit $B$
$C=$ Stockdale Unit $C$

CHICK = Chickamauga Group

CON = Conasauga Group

COPPER RIDGE = Copper Ridge

Dolomite

D = Stockdale Unit D

$E$ = Stockdale Unit E

$\mathbf{G}=$ Stockdale Unit $\mathbf{G}$

KNOX = Knox Group

MARYVILLE = Maryville Limestone

MAYNARDVILLE = Maynardville

Limestone

NOLICHICKY $=$ Nolichicky Shale

PUMPKIN VALLEY = Pumpkin

Valley Shale

ROME = Rome Formation

ROGERSVILLE = Rogersville Shale

RUTLEDGE = Rutlege Limestone

Coordinate grid system for the well location

Hydraulic conductivity (m/d)

Indicates whether any geologic or geophysical logs were obtained

$\mathrm{T}=$ True, $\mathrm{a}$ log was obtained

$F=$ False, a log was not obtained 
Table 5.5 (continued)

\begin{tabular}{|c|c|c|c|c|}
\hline $\begin{array}{l}\text { Variable } \\
\text { name }\end{array}$ & Type & Width & $\begin{array}{l}\text { Decimal } \\
\text { places }\end{array}$ & Description \\
\hline NORTH & Numeric & 16 & 4 & $\begin{array}{l}\text { North coordinate. Coordinate grid system } \\
\text { is found in the GRID variable }\end{array}$ \\
\hline OPEN_SCR & Character & 10 & & $\begin{array}{l}\text { Indicates whether the well has an open } \\
\text { or screened interval } \\
\text { OPEN = an open interval } \\
\text { SCREENED = a screened interval } \\
\text { UNKNOWN = it is not possible to } \\
\text { determine from the source data base if } \\
\text { the interval is open or screened } \\
\text { OTHER = a well that does not fall } \\
\text { into the previous categories }\end{array}$ \\
\hline PLANT & Character & 20 & & $\begin{array}{l}\text { Plant that installed the well (key) } \\
\begin{aligned} \mathrm{K} 25 & =\mathrm{K}-25 \\
\mathrm{X} 10 & =\mathrm{ORNL} \\
\mathrm{Y} 12 & =\mathrm{Y}-12\end{aligned}\end{array}$ \\
\hline ROCTOP & Numeric & 16 & 4 & Depth to the top of rock $(\mathrm{ft})$ \\
\hline ROCTOPFR & Numeric & 16 & 4 & Depth to the top of fresh rock (ft) \\
\hline ROCTOPWT & Numeric & 16 & 4 & Depth to the top of weathered rock (ft) \\
\hline SITE & Character & 27 & & $\begin{array}{l}\text { Name of the site where the well is } \\
\text { located }\end{array}$ \\
\hline
\end{tabular}


Table 5.5 (continued)

\begin{tabular}{|c|c|c|c|c|}
\hline $\begin{array}{l}\text { Variable } \\
\text { name }\end{array}$ & Type & Width & $\begin{array}{l}\text { Decimal } \\
\text { places }\end{array}$ & Description \\
\hline SRC_GEN & Character & 30 & & $\begin{array}{l}\text { Source of the data } \\
\text { The plant the data came from is listed } \\
\text { first } \\
\text { K25 = K-25 } \\
\text { X10 = ORNL } \\
\text { Y12 = Y-12 } \\
\text { The type of the original data file is listed } \\
\text { second } \\
\text { CHEM = a groundwater analyses file } \\
\text { WELL = a well construction file } \\
\text { The original data file name is listed last, } \\
\text { in parentheses }\end{array}$ \\
\hline TRANS & Numeric & 16 & 4 & Transmissivity $\left(\mathrm{m}^{2} / \mathrm{d}\right)$ \\
\hline WAG & Character & 10 & & Name of the waste area grouping \\
\hline WELALT_1 & Character & 20 & & First alternate well name \\
\hline WELTYP_1 & Character & 20 & & First description of well type \\
\hline WELTYP_2 & Character & 40 & & Second description of well type \\
\hline WELL & Character & 20 & & Official well name (key) \\
\hline
\end{tabular}


Table 5.6. Description of METAL file

Variable name

Type

Numeric $16 \quad 4$

Decimal

Width places Description

\begin{tabular}{|c|c|c|c|c|}
\hline ALUM_I & Numeric & 16 & 4 & $\begin{array}{l}\text { Concentration of aluminum (ICAP, } \\
\mathrm{mg} / \mathrm{L} \text { ) }\end{array}$ \\
\hline ALUM_I_C & Character & 4 & & $\begin{array}{l}\text { Laboratory qualifiers for the } \\
\text { concentration of aluminum (ICAP) }\end{array}$ \\
\hline ANT_I & Ninmeric & 16 & 4 & Concentration of antimony (ICAP, $\mathrm{mg} / \mathrm{L}$ ) \\
\hline ANT_I_C & Character & 4 & & $\begin{array}{l}\text { Laboratory qualifiers for the } \\
\text { concentration of antimony (ICAP) }\end{array}$ \\
\hline ARSN_A & Numeric & 16 & 4 & Concentration of arsenic (AAS, mg/L) \\
\hline AFSN_A_C & Character & 4 & & $\begin{array}{l}\text { Iaboratory qualifiers for the } \\
\text { concentration of arsenic (AAS) }\end{array}$ \\
\hline ARSN_I & Numeric & 16 & 4 & Concentration of arsenic (ICAP, mg/L) \\
\hline ARSN_I C & Character & 4 & & $\begin{array}{l}\text { Laboratory qualifiers for the } \\
\text { concentration of arsenic (ICAP) }\end{array}$ \\
\hline PARI_Á & Numeric & 16 & 4 & Concentration of barium (AAS, mg/L) \\
\hline BARI_A_C & Character & 4 & & $\begin{array}{l}\text { Laboratory qualifiers for the } \\
\text { concentration of barium (AAS) }\end{array}$ \\
\hline BARI_I & Numeric & 16 & 4 & Concentration of barium (ICAP, mg/L) \\
\hline 3ARI_I_C & Character & 4 & & $\begin{array}{l}\text { Laboratory qualifiers for the } \\
\text { concentration of barium (ICAP) }\end{array}$ \\
\hline BERY_A & Numeric & 16 & 4 & Concentration of beryllium (AAS, mg/L) \\
\hline BERY_A_C & Character & 4 & & $\begin{array}{l}\text { Laboratory qualifiers for the } \\
\text { concentration of beryllium (AAS) }\end{array}$ \\
\hline BERY_I & Numeric & 16 & 4 & Concentration of beryllium (ICAP, mg/L) \\
\hline BERY_I_C & Character & 4 & & $\begin{array}{l}\text { Laboratory qualifiers for the } \\
\text { concentration of beryllium (ICAP) }\end{array}$ \\
\hline
\end{tabular}


Table 5.6 (continued)

Variable name

Type

Decimal

Width places Description

\begin{tabular}{|c|c|c|c|c|}
\hline BOR_I & Numeric & 16 & 4 & Concentration of boron (ICAP, mg/L) \\
\hline BOR_I_C & Character & 4 & & $\begin{array}{l}\text { Laboratory qualifiers for the } \\
\text { concestration of boron (ICAP) }\end{array}$ \\
\hline CADM_A & Numeric & 16 & 4 & Concentration of cadmium (AAS, mg/L) \\
\hline CADM_A_C & Character & 4 & & $\begin{array}{l}\text { Laboratory qualifiers for the } \\
\text { concentration of cadmium (AAS) }\end{array}$ \\
\hline CADM_I & Numeric & 16 & 4 & Concentration of cadmium (ICAP, mg/L) \\
\hline CADM_I_C & Character & 4 & & $\begin{array}{l}\text { Laboratory qualifiers for the } \\
\text { concentration of cadmium (ICAP) }\end{array}$ \\
\hline CADM_P & Numeric & 16 & 4 & Concentration of cadmium (Polar, mg/L) \\
\hline CADM_P_C & Character & 4 & & $\begin{array}{l}\text { Laboratory qualifiers for the } \\
\text { concentration of cadmium (Polar) }\end{array}$ \\
\hline CALC_I & Numeric & 16 & 4 & Concentration of calcium (ICAP, my/L) \\
\hline CALC_I_C & Character & 4 & & $\begin{array}{l}\text { Laboratory qualifiers for the } \\
\text { concentration of calcium (ICAP) }\end{array}$ \\
\hline CHRO_A & Numeric & 16 & 4 & Concentration of chromium (AAS, $\mathrm{mg} / \mathrm{L}$ ) \\
\hline CHRO_A_C & Character & 4 & & $\begin{array}{l}\text { Laboratory qualifiers for the } \\
\text { concentration of chromium (AAS) }\end{array}$ \\
\hline CHRO_I & Numeric & 16 & 4 & $\begin{array}{l}\text { Concentration of chromium (ICAP, } \\
\mathrm{mg} / \mathrm{L} \text { ) }\end{array}$ \\
\hline CHRO_I_C & Character & 4 & & $\begin{array}{l}\text { Laboratory qualifiers for the } \\
\text { concentration of chromium (ICAP) }\end{array}$ \\
\hline CHRO_P & Numeric & 16 & 4 & Concentration of chromiurn (Polar, $\mathrm{mg} / \mathrm{L}$ ) \\
\hline CHRO_P_C & Character & 4 & & $\begin{array}{l}\text { Laboratory qualifiers for the } \\
\text { concentration of chromium (Polar) }\end{array}$ \\
\hline
\end{tabular}


Table 5.6 (continued)

\begin{tabular}{|c|c|c|c|c|}
\hline $\begin{array}{l}\text { Variable } \\
\text { name }\end{array}$ & Type & Width & $\begin{array}{l}\text { Decimal } \\
\text { places }\end{array}$ & Description \\
\hline COBL_I & Numeric & 16 & 4 & Concentration of cobalt (ICAP, mg/L) \\
\hline COBL_I_C & Character & 4 & & $\begin{array}{l}\text { Laboratory qualifiers for the } \\
\text { concentration of cobalt (ICAP) }\end{array}$ \\
\hline COPP_I & Numeric & 16 & 4 & Concentration of copper (ICAP, mg/L) \\
\hline COPP_I_C & Character & 4 & & $\begin{array}{l}\text { Laboratory qualifiers for the } \\
\text { concentration of copper (ICAP) }\end{array}$ \\
\hline DATE_SAM & Date & 8 & & $\begin{array}{l}\text { Date the groundwater sample was taken } \\
\text { (key) }\end{array}$ \\
\hline DUP_Q & Logical & 1 & & $\begin{array}{l}\text { Whether it is a duplicate sample (key) } \\
\qquad \begin{array}{l}T=\text { True, it is a duplicate sample } \\
F=\text { False, it is not a duplicate sample }\end{array}\end{array}$ \\
\hline FILTER_Q & Logical & 1 & & $\begin{array}{l}\text { Whether it is a filtered sample (key) } \\
\qquad \begin{array}{l}\mathrm{T}=\text { True, it is a filtered sample } \\
\mathrm{F}=\text { False, it is not a filtered sample }\end{array}\end{array}$ \\
\hline GALI_I & Numeric & 16 & 4 & Concentration of gallium (ICAP, mg/L) \\
\hline GALI_I_C & Character & 4 & & $\begin{array}{l}\text { Laboratory qualifiers for the } \\
\text { concentration of gallium }\end{array}$ \\
\hline IRON_A & Numeric & 16 & 4 & Concentration of iron (AAS, mg/L) \\
\hline IRON_A_C & Character & 4 & & $\begin{array}{l}\text { Laboratory qualifiers for the } \\
\text { concentration of iron }\end{array}$ \\
\hline IRON_I & Numeric & 16 & 4 & Concentration of iron (ICAP, $\mathrm{mg} / \mathrm{L}$ ) \\
\hline IRON_I_C & Character & 4 & & $\begin{array}{l}\text { Laboratory qualifiers for the } \\
\text { concentration of iron (ICAP) }\end{array}$ \\
\hline LEAD_A & Numeric & 16 & 4 & Concentration of lead (AAS, mg/L) \\
\hline LEAD_A_C & Character & 4 & & $\begin{array}{l}\text { Laboratory qualifiers for the } \\
\text { concentration of lead (AAS) }\end{array}$ \\
\hline
\end{tabular}


Table 5.6 (continued)

\begin{tabular}{|c|c|c|c|c|}
\hline $\begin{array}{l}\text { Variable } \\
\text { name }\end{array}$ & Type & Width & $\begin{array}{l}\text { Decimal } \\
\text { places }\end{array}$ & Description \\
\hline LEAD_P & Numeric & 16 & 4 & Concentration of lead (Polar, $\mathrm{mg} / \mathrm{L}$ ) \\
\hline LEAD_P_C & Character & 4 & & $\begin{array}{l}\text { Laboratory qualifiers for the } \\
\text { concentration of lead (Polar) }\end{array}$ \\
\hline LTTH_I & Numeric & 16 & 4 & Concentration of lithium (ICAP, mg/L) \\
\hline LITH_I_C & Character & 4 & & $\begin{array}{l}\text { Laboratory qualifiers for the } \\
\text { concentration of lithium (ICAP) }\end{array}$ \\
\hline MAGN_I & Numeric & 16 & 4 & $\begin{array}{l}\text { Concentration of magnesium (ICAP, } \\
\mathrm{mg} / \mathrm{L} \text { ) }\end{array}$ \\
\hline MAGN_I_C & Character & 4 & & $\begin{array}{l}\text { Laboratory qualifiers for the } \\
\text { concentration of magnesium (ICAP) }\end{array}$ \\
\hline MANG_A & Numeric & 16 & 4 & $\begin{array}{l}\text { Concentration of manganese (AAS, } \\
\mathrm{mg} / \mathrm{L} \text { ) }\end{array}$ \\
\hline MANG_A_C & Character & 4 & & $\begin{array}{l}\text { Laboratory qualifiers for the } \\
\text { concentration of manganese (AAS) }\end{array}$ \\
\hline MANG_I & Numeric & 16 & 4 & $\begin{array}{l}\text { Concentration of manganese (ICAP, } \\
\mathrm{mg} / \mathrm{L})\end{array}$ \\
\hline MANG_I_C & Character & 4 & & $\begin{array}{l}\text { Laboratory qualifiers for the } \\
\text { concentration of manganese (ICAP) }\end{array}$ \\
\hline MERC_V & Numeric & 16 & 4 & Concentration of mercury (CVAA, mg/L) \\
\hline MERC_V_C & Character & 4 & & $\begin{array}{l}\text { Laboratory qualifiers for the } \\
\text { concentration of mercury (CVAA) }\end{array}$ \\
\hline MOLY_I & Numeric & 16 & 4 & $\begin{array}{l}\text { Concentration of molybdenum (ICAP, } \\
\mathrm{mg} / \mathrm{L})\end{array}$ \\
\hline MOLY_I_C & Character & 4 & & $\begin{array}{l}\text { Laboratory qualifiers for the } \\
\text { concentration of molybdenum (ICAP) }\end{array}$ \\
\hline NICK_I & Numeric & 16 & 4 & Concentration of nickel (ICAP, mg/L) \\
\hline
\end{tabular}


Table 5.6 (continued)

\begin{tabular}{|c|c|c|c|c|}
\hline $\begin{array}{l}\text { Variable } \\
\text { name }\end{array}$ & Type & Width & $\begin{array}{l}\text { Decimal } \\
\text { places }\end{array}$ & Description \\
\hline NICK_I_C & Character & 4 & & $\begin{array}{l}\text { Laboratory qualifiers for the } \\
\text { concentration of nickel (ICAP) }\end{array}$ \\
\hline NIOB_I & Numeric & 16 & 4 & Concentration of niobium (ICAP, mg/L) \\
\hline NIOB_I_C & Character & 4 & & $\begin{array}{l}\text { Laboratory qualifiers for the } \\
\text { concentration of niobium (ICAP) }\end{array}$ \\
\hline PHOS_I & Numeric & 16 & 4 & $\begin{array}{l}\text { Concentration of phosphorus (ICAP, } \\
\mathrm{mg} / \mathrm{L} \text { ) }\end{array}$ \\
\hline PHOS_I_C & Character & 4 & & $\begin{array}{l}\text { Laboratory qualifiers for the } \\
\text { concentration of phosphorus (ICAP) }\end{array}$ \\
\hline PLANT & Character & 3 & & $\begin{array}{l}\text { Plant that installed the well (key) } \\
\begin{aligned} \mathrm{K} 25 & =\mathrm{K}-25 \\
\mathrm{X} 10 & =\mathrm{ORNL} \\
\mathrm{Y} 12 & =\mathrm{Y}-12\end{aligned}\end{array}$ \\
\hline POTA_E & Numeric & 16 & 4 & $\begin{array}{l}\text { Concentration of potassium } \\
\text { (Flame - emission, mg/L) }\end{array}$ \\
\hline POTA_E_C & Character & 4 & & $\begin{array}{l}\text { Laboratory qualifiers for the concentration } \\
\text { of potassium (Flame - emission) }\end{array}$ \\
\hline POTA_I & Numeric & 16 & 4 & Concentration of potassium (ICAP, $\mathrm{mg} / \mathrm{L}$ ) \\
\hline POTA_I_C & Character & 4 & & $\begin{array}{l}\text { Laboratory qualifiers for the concentration } \\
\text { of potassium (ICAP) }\end{array}$ \\
\hline SELE_A & Numeric & 16 & 4 & Concentration of selenium (AAS, mg/L) \\
\hline SELE_A_C & Character & 4 & & $\begin{array}{l}\text { Laboratory qualifiers for the concentration } \\
\text { of selenium (AAS) }\end{array}$ \\
\hline SELE_I & Numeric & 16 & 4 & Concentration of selenium (ICAP, $\mathrm{mg} / \mathrm{L}$ ) \\
\hline SELE_I_C & Character & 4 & & $\begin{array}{l}\text { Laboratory qualifiers for the concentration } \\
\text { of selenium (ICAP) }\end{array}$ \\
\hline
\end{tabular}


Table 5.6 (continued)

\begin{tabular}{|c|c|c|c|c|}
\hline $\begin{array}{l}\text { Variable } \\
\text { name }\end{array}$ & Type & Width & $\begin{array}{l}\text { Decimal } \\
\text { places }\end{array}$ & Description \\
\hline SILI_I & Numeric & 16 & 4 & Concentration of silicon (ICAP, $\mathrm{mg} / \mathrm{L}$ ) \\
\hline SILI_I_C & Character & 4 & & $\begin{array}{l}\text { Laboratory qualifiers for the } \\
\text { concentration of silicon (ICAP) }\end{array}$ \\
\hline SILV_A & Numeric & 16 & 4 & Concentration of silver (AAS, mg/L) \\
\hline SILV_A_C & Character & 4 & & $\begin{array}{l}\text { Laboratory qualifiers for the } \\
\text { concentration of silver (AAS) }\end{array}$ \\
\hline SILV_I & Numeric & 16 & 4 & Concentration of silver (ICAP, mg/L) \\
\hline SILV_I_C & Character & 4 & & $\begin{array}{l}\text { Laboratory qualifiers for the } \\
\text { concentration of silver (ICAP) }\end{array}$ \\
\hline SODI_A & Numeric & 16 & 4 & Concentration of sodium (AAS, $\mathrm{mg} / \mathrm{L}$ ) \\
\hline SODI_A_C & Character & 4 & & $\begin{array}{l}\text { Laboratory qualifiers for the } \\
\text { concentration of sodium (AAS) }\end{array}$ \\
\hline SODI_I & Numeric & 16 & 4 & Concentration of sodium (ICAP, mg/L) \\
\hline SODI_I_C & Character & 4 & & $\begin{array}{l}\text { Laboratory qualifiers for the } \\
\text { concentration of sodium (ICAP) }\end{array}$ \\
\hline SRC_MET & Character & 30 & & $\begin{array}{l}\text { Source of the data } \\
\text { The plant the data came from is listed } \\
\text { first } \\
\text { K25 }=\mathrm{K}-25 \\
\mathrm{X} 10=\mathrm{ORNL} \\
\text { Y12 }=\text { Y-12 } \\
\text { The type of the original data file is listed } \\
\text { second } \\
\text { CHEM = a groundwater analyses file } \\
\text { WELL = a well construction file } \\
\text { The original data file name is listed last, } \\
\text { in parentheses }\end{array}$ \\
\hline
\end{tabular}

STRO_I Numeric $16 \quad 4 \quad$ Concentration of strontium (ICAP, mg/L) 
Table 5.6 (continued)

Variable name

Type

Decimal

Width places Description

\begin{tabular}{|c|c|c|c|c|}
\hline STRO_I_C & Character & 4 & & $\begin{array}{l}\text { Laboratory qualifiers for the } \\
\text { concentration of strontium (ICAP) }\end{array}$ \\
\hline THAL_A & Numeric & 16 & 4 & Concentration of thallium (AAS, $\mathrm{mg} / \mathrm{L}$ ) \\
\hline THAL_A_C & Character & 4 & & $\begin{array}{l}\text { Laboratory qualifiers for the } \\
\text { concentration of thallium (AAS) }\end{array}$ \\
\hline THOR_I & Numeric & 16 & 4 & Concentration of thorium (ICAP, mg/L) \\
\hline THOR_I_C & Character & 4 & & $\begin{array}{l}\text { Laboratory qualifiers for the } \\
\text { concentration of thorium (ICAP) }\end{array}$ \\
\hline TIN_A & Numeric & 16 & 4 & Concentration of tin (AAS, mg/L) \\
\hline TIN_A_C & Character & 4 & & $\begin{array}{l}\text { Laboratory qualifiers for the } \\
\text { concentration of tin (AAS) }\end{array}$ \\
\hline TIN_I & Numeric & 16 & 4 & Concentration of tin (ICAP, mg/L) \\
\hline TIN_I_C & Character & 4 & & $\begin{array}{l}\text { Laboratory qualifiers for the } \\
\text { concentration of tin (ICAP) }\end{array}$ \\
\hline TITA_I & Numeric & 16 & 4 & Concentration of titanium (ICAP, mg/L) \\
\hline TITA_I_C & Character & 4 & & $\begin{array}{l}\text { Laboratory qualifiess for the } \\
\text { concentration of titanium (ICAP) }\end{array}$ \\
\hline URAN_F & Numeric & 16 & 4 & $\begin{array}{l}\text { Concentration of uranium (fluorometric, } \\
\mathrm{mg} / \mathrm{L} \text { ) }\end{array}$ \\
\hline URAN_F_C & Character & 4 & & $\begin{array}{l}\text { Laboratory qualifiers for the } \\
\text { concentration of uranium (fluorometric) }\end{array}$ \\
\hline URAN_I & Numeric & 16 & 4 & Concentration of uranium (ICAP, $\mathrm{mg} / \mathrm{L}$ ) \\
\hline URAN_I_C & Character & 4 & & $\begin{array}{l}\text { Laboratory qualifiers for the } \\
\text { concentration of uranium (ICAP) }\end{array}$ \\
\hline VANA_I & Numeric & 16 & 4 & Concentration of vanadium (ICAP, $\mathrm{mg} / \mathrm{L}$ ) \\
\hline
\end{tabular}


Table 5.6 (continued)

\begin{tabular}{|c|c|c|c|c|}
\hline $\begin{array}{l}\text { Variable } \\
\text { name }\end{array}$ & Type & Width & $\begin{array}{l}\text { Decimal } \\
\text { places }\end{array}$ & Description \\
\hline VANA_I_C & Character & 4 & & $\begin{array}{l}\text { Laboratory qualifiers for the } \\
\text { concentration of vanadium (ICAP) }\end{array}$ \\
\hline WELL & Character & 20 & & Official well name (key) \\
\hline ZINC_I & Numeric & 16 & 4 & Concentration of zinc (ICAP, $\mathrm{mg} / \mathrm{L}$ ) \\
\hline ZINC_I_C & Character & 4 & & $\begin{array}{l}\text { Laboratory qualifiers for the } \\
\text { concentration of zinc (ICAP) }\end{array}$ \\
\hline ZIRC_I & Numeric & 16 & 4 & $\begin{array}{l}\text { Concentration of zirconium (ICAP, } \\
\mathrm{mg} / \mathrm{L} \text { ) }\end{array}$ \\
\hline ZIRC_I_C & Character & 4 & & $\begin{array}{l}\text { Laboratory qualifiers for the } \\
\text { concentration of zirconium (ICAP) }\end{array}$ \\
\hline
\end{tabular}


Table 5.7. Description of MISC file

\begin{tabular}{|c|c|c|c|c|}
\hline $\begin{array}{l}\text { Variable } \\
\text { name }\end{array}$ & Type & Width & $\begin{array}{l}\text { Decimal } \\
\text { places }\end{array}$ & Description \\
\hline AMMONI & Numeric & 16 & 4 & Concentration of ammonia (as $\mathrm{N}, \mathrm{mg} / \mathrm{L}$ ) \\
\hline AMMONI_C & Character & 4 & & $\begin{array}{l}\text { Laboratory qualifiers for the } \\
\text { concentration of ammonia }\end{array}$ \\
\hline BIOXD & Numeric & 16 & 4 & $\begin{array}{l}\text { Concentration of biochemical oxygen } \\
\text { demand (mg/L) }\end{array}$ \\
\hline BIOXD_C & Character & 4 & & $\begin{array}{l}\text { Laboratory qualifiers for the } \\
\text { concentration of biochemical oxygen } \\
\text { demand }\end{array}$ \\
\hline CHMOXD & Numeric & 16 & 4 & $\begin{array}{l}\text { Concentration of chemical oxygen } \\
\text { demand }(m g / L)\end{array}$ \\
\hline CHMOXD_C & Character & 4 & & $\begin{array}{l}\text { Laboratory qualifiers for the } \\
\text { concentration of chemical oxygen } \\
\text { demand }\end{array}$ \\
\hline COLIFO & Numeric & 16 & 4 & $\begin{array}{l}\text { Concentration of total coliform } \\
\text { (colonies } / 100 \mathrm{~mL} \text { ) }\end{array}$ \\
\hline COLIFO_C & Character & 4 & & $\begin{array}{l}\text { Laboratory qualifiers for the } \\
\text { concentration of total coliform }\end{array}$ \\
\hline CON_L1 & Numeric & 16 & 4 & $\begin{array}{l}\text { First laboratory specific conductance of } \\
\text { water }(\mu \mathrm{mhos} / \mathrm{cm})\end{array}$ \\
\hline CON_L1_C & Character & 4 & & $\begin{array}{l}\text { Laboratory qualifiers for the first } \\
\text { laboratory specific conductance of water }\end{array}$ \\
\hline CON_L2 & Numeric & 16 & 4 & $\begin{array}{l}\text { Second laboratory specific conductance } \\
\text { of water }(\mu \mathrm{mhos} / \mathrm{cm})\end{array}$ \\
\hline CON_L2_C & Character & 4 & & $\begin{array}{l}\text { Laboratory qualifiers for the second } \\
\text { laboratory specific conductance of water }\end{array}$ \\
\hline CON_L3 & Numeric & 16 & 4 & $\begin{array}{l}\text { Third laboratory specific conductance of } \\
\text { water }(\mu \mathrm{mhos} / \mathrm{cm})\end{array}$ \\
\hline
\end{tabular}


Table 5.7 (continued)

\begin{tabular}{|c|c|c|c|c|}
\hline $\begin{array}{l}\text { Variable } \\
\text { name }\end{array}$ & Type & Width & $\begin{array}{l}\text { Decimal } \\
\text { places }\end{array}$ & Description \\
\hline CON_L3_C & Character & 4 & & $\begin{array}{l}\text { Laboratory qualifiers for the third } \\
\text { laboratory specific conductance of water }\end{array}$ \\
\hline CON_LA & Numeric & 16 & 4 & $\begin{array}{l}\text { Fourth laboratory specific conductance of } \\
\text { water }(\mu \mathrm{mhos} / \mathrm{cm})\end{array}$ \\
\hline CON_LA_C & Character & 4 & & $\begin{array}{l}\text { Laboratory qualifiers for the fourth } \\
\text { laboratory specific conductance of water }\end{array}$ \\
\hline DATE_SAM & Date & 8 & & $\begin{array}{l}\text { Date the groundwater sample was taken } \\
\text { (key) }\end{array}$ \\
\hline DUP_Q & Logical & 1 & & $\begin{array}{l}\text { Whether it is a duplicate sample (key) } \\
\qquad \begin{array}{l}\mathrm{T}=\text { True, it is a duplicate sample } \\
\mathrm{F}=\text { False, it is not a duplicate sample }\end{array}\end{array}$ \\
\hline FECCOL & Numeric & 16 & 4 & $\begin{array}{l}\text { Concentration of fecal coliform } \\
\text { (col/100 ML) }\end{array}$ \\
\hline FECCOL_C & Character & 4 & & $\begin{array}{l}\text { Laboratory qualifiers for the concentration } \\
\text { of fecal coliform }\end{array}$ \\
\hline FILTER_Q & Logical & 1 & & $\begin{array}{l}\text { Whether it is a filtered sample (key) } \\
\qquad \begin{array}{l}T=\text { True, it is a filtered sample } \\
F=\text { False, it is not a filtered sample }\end{array}\end{array}$ \\
\hline HARD & Numeric & 16 & 4 & Concentration of hardness (mg/L) \\
\hline HARD_C & Character & 4 & & $\begin{array}{l}\text { Laboratory qualifiers for the concentration } \\
\text { of hardness }\end{array}$ \\
\hline HYDROX & Numeric & 16 & 4 & Concentration of hydroxyl (mg/L) \\
\hline HYDROX_C & Character & 4 & & $\begin{array}{l}\text { Laboratory qualifiers for the concentration } \\
\text { of hydroxyl }\end{array}$ \\
\hline NIT_KJ & Numeric & 16 & 4 & $\begin{array}{l}\text { Concentration of total Kjeldahl nitrogen } \\
(\mathrm{mg} / \mathrm{L})\end{array}$ \\
\hline
\end{tabular}


Table 5.7 (continued)

\begin{tabular}{|c|c|c|c|c|}
\hline $\begin{array}{l}\text { Variable } \\
\text { name }\end{array}$ & Type & Width & $\begin{array}{l}\text { Decimal } \\
\text { places }\end{array}$ & Description \\
\hline NIT_KJ_C & Character & 4 & & $\begin{array}{l}\text { Laboratory qualifiers for the } \\
\text { concentration of total Kjeldahl nitrogen }\end{array}$ \\
\hline PH_L1 & Numeric & 16 & 4 & First laboratory pH (standard units) \\
\hline PH_L1_C & Character & 4 & & $\begin{array}{l}\text { Laboratory qualifiers for the first } \\
\text { laboratory } \mathrm{pH}\end{array}$ \\
\hline PH_L2 & Numeric & 16 & 4 & Second laboratory pH (standard units) \\
\hline PH_L2_C & Character & 4 & & $\begin{array}{l}\text { Laboratory qualifiers for the second } \\
\text { laboratory } \mathrm{pH}\end{array}$ \\
\hline PH_L3 & Numeric & 16 & 4 & Third laboratory pH (standard units) \\
\hline PH_L3_C & Character & 4 & & $\begin{array}{l}\text { Laboratory qualifiers for the third } \\
\text { laboratory } \mathrm{pH}\end{array}$ \\
\hline PH_LA & Numeric & 16 & 4 & Fourth laboratory $\mathrm{pH}$ (standard units) \\
\hline PH_LA_C & Character & 4 & & $\begin{array}{l}\text { Laboratory qualifiers for the fourth } \\
\text { laboratory } \mathrm{pH}\end{array}$ \\
\hline PHN_TL & Numeric & 16 & 4 & Concentration of total phenols $(\mathrm{mg} / \mathrm{L})$ \\
\hline PHN_TL_C & Character & 4 & & $\begin{array}{l}\text { Laboratory qualifiers for the } \\
\text { concentration of total phenols }\end{array}$ \\
\hline PLANT & Character & 3 & & $\begin{array}{l}\text { Plant that installed the well (key) } \\
\text { K25 }=\mathrm{K}-25 \\
\mathrm{X} 10=\mathrm{ORNL} \\
\mathrm{Y} 12=\mathrm{Y}-12\end{array}$ \\
\hline
\end{tabular}


Table 5.7 (continued)

\begin{tabular}{|c|c|c|c|c|}
\hline $\begin{array}{l}\text { Variable } \\
\text { name }\end{array}$ & Type & Width & $\begin{array}{l}\text { Decimal } \\
\text { places }\end{array}$ & Description \\
\hline SRC_MISC & Character & 30 & & $\begin{array}{l}\text { Source of the data } \\
\text { The plant the data came from is listed } \\
\text { first } \\
\text { K25 = K-25 } \\
\text { X10 = ORNL } \\
\text { Y12 = Y-12 } \\
\text { The type of the original data file is listed } \\
\text { second } \\
\text { CHEM = a groundwater analyses file } \\
\text { WELL = a well construction file } \\
\text { The original data file name is listed last, } \\
\text { in parentheses }\end{array}$ \\
\hline TL_DIS & Numeric & 16 & 4 & $\begin{array}{l}\text { Concentration of total dissolved solids } \\
(\mathrm{mg} / \mathrm{L})\end{array}$ \\
\hline TL_DIS_C & Character & 4 & & $\begin{array}{l}\text { Laboratory qualifiers for the } \\
\text { concentration of total dissolved solids }\end{array}$ \\
\hline TL_SUS & Numeric & 16 & 4 & $\begin{array}{l}\text { Concentration of total suspended solids } \\
(\mathrm{mg} / \mathrm{L})\end{array}$ \\
\hline TL_SUS_C & Character & 4 & & $\begin{array}{l}\text { Laboratory qualifiers for the } \\
\text { concentration of total suspended solids }\end{array}$ \\
\hline TOC_1 & Numeric & 16 & 4 & $\begin{array}{l}\text { Concentration of the first total organic } \\
\text { carbon }(\mathrm{mg} / \mathrm{L})\end{array}$ \\
\hline TOC_1_C & Character & 4 & & $\begin{array}{l}\text { Laboratory ualifiers for the } \\
\text { concentration of the first total organic } \\
\text { carbon }\end{array}$ \\
\hline TOC_2 & Numeric & 16 & 4 & $\begin{array}{l}\text { Concentration of the second total organic } \\
\text { carbon }(\mathrm{mg} / \mathrm{L})\end{array}$ \\
\hline TOC_2_C & Character & 4 & & $\begin{array}{l}\text { Laboratory qualifiers for the } \\
\text { concentration of the second total organic } \\
\text { carbon }\end{array}$ \\
\hline
\end{tabular}


Table 5.7 (continued)

\begin{tabular}{|c|c|c|c|c|}
\hline $\begin{array}{l}\text { Variable } \\
\text { name }\end{array}$ & Type & Width & $\begin{array}{l}\text { Decimal } \\
\text { places }\end{array}$ & Description \\
\hline TOC_3 & Numeric & 16 & 4 & $\begin{array}{l}\text { Concentration of the third total organic } \\
\text { carbon }(\mathrm{mg} / \mathrm{L})\end{array}$ \\
\hline TOC_3_C & Character & 4 & & $\begin{array}{l}\text { Laboratory qualifiers for the } \\
\text { concentration of the third total organic } \\
\text { carbon }\end{array}$ \\
\hline TOC_4 & Numeric & 16 & 4 & $\begin{array}{l}\text { Concentration of the fourth total organic } \\
\text { carbon }(\mathrm{mg} / \mathrm{L})\end{array}$ \\
\hline TOC_4_C & Character & 4 & . & $\begin{array}{l}\text { Laboratory qualifiers for the } \\
\text { concentration of the fourth total organic } \\
\text { carbon }\end{array}$ \\
\hline TOCL_1 & Numeric & 16 & 4 & $\begin{array}{l}\text { Concentration of the first total organic } \\
\text { chloride }(\mu \mathrm{g} / \mathrm{L})\end{array}$ \\
\hline TOCL_1_C & Character & 4 & & $\begin{array}{l}\text { Laboratory qualifiers for the } \\
\text { concentration of the first total organic } \\
\text { chloride }\end{array}$ \\
\hline TOCL_2 & Numeric & 16 & 4 & $\begin{array}{l}\text { Concentration of the second total organic } \\
\text { chloride }(\mu \mathrm{g} / \mathrm{L})\end{array}$ \\
\hline TOCL_2_C & Character & 4 & & $\begin{array}{l}\text { Laboratory qualifiers for the } \\
\text { concentration of the second total organic } \\
\text { chloride }\end{array}$ \\
\hline TOCL_3 & Numeric & 16 & 4 & $\begin{array}{l}\text { Concentration of the third total organic } \\
\text { chloride }(\mu \mathrm{g} / \mathrm{L})\end{array}$ \\
\hline TOCL_3_C & Character & 4 & & $\begin{array}{l}\text { Laboratory qualifiers for the } \\
\text { concentration of the third total organic } \\
\text { chloride }\end{array}$ \\
\hline TOCL_4 & Numeric & 16 & 4 & $\begin{array}{l}\text { Concentration of the fourth total organic } \\
\text { chloride }(\mu \mathrm{g} / \mathrm{L})\end{array}$ \\
\hline
\end{tabular}


Table 5.7 (continued)

\begin{tabular}{|c|c|c|c|c|}
\hline $\begin{array}{l}\text { Variable } \\
\text { name }\end{array}$ & Type & Width & $\begin{array}{l}\text { Decimal } \\
\text { places }\end{array}$ & Description \\
\hline TOCL_4_C & Character & 4 & & $\begin{array}{l}\text { Laboratory qualifiers for the } \\
\text { concentration of the fourth total organic } \\
\text { chloride }\end{array}$ \\
\hline TOX_1 & Numeric & 16 & 4 & $\begin{array}{l}\text { Concentration of the first total organic } \\
\text { halides }(\mu \mathrm{g} / \mathrm{L})\end{array}$ \\
\hline TOX_1_C & Character & 4 & & $\begin{array}{l}\text { Laboratory qualifiers for the } \\
\text { concentration of the first total organic } \\
\text { halides }\end{array}$ \\
\hline TOX_2 & Numeric & 16 & 4 & $\begin{array}{l}\text { Concentration of the second total organic } \\
\text { halides }(\mu \mathrm{g} / \mathrm{L})\end{array}$ \\
\hline TOX_2_C & Character & 4 & & $\begin{array}{l}\text { Laboratory qualifiers for the } \\
\text { concentration of the second total organic } \\
\text { halides }\end{array}$ \\
\hline TOX_3 & Numeric & 16 & 4 & $\begin{array}{l}\text { Concentration of the third total organic } \\
\text { halides }(\mu \mathrm{g} / \mathrm{L})\end{array}$ \\
\hline TOX_3_C & Character & 4 & & $\begin{array}{l}\text { Laboratory qualifiers for the } \\
\text { concentration of the third total organic } \\
\text { halides }\end{array}$ \\
\hline TOX_4 & Numeric & 16 & 4 & $\begin{array}{l}\text { Concentration of the fourth total organic } \\
\text { halides }(\mu \mathrm{g} / \mathrm{L})\end{array}$ \\
\hline TOX_4_C & Character & 4 & & $\begin{array}{l}\text { Laboratory qualifiers for the } \\
\text { concentration of the fourth total organic } \\
\text { halides }\end{array}$ \\
\hline TURBID & Numeric & 16 & 4 & Turbidity (NTU) \\
\hline TURBID_C & Character & 4 & & Laboratory qualifiers for turbidity \\
\hline WAT_LEV & Numeric & 16 & 4 & Water level ( $f t$ ) below measuring point \\
\hline WELL & Character & 20 & & Official well name (key) \\
\hline
\end{tabular}


Table 5.8 Description of PCB file

\begin{tabular}{|c|c|c|c|c|}
\hline $\begin{array}{l}\text { Variable } \\
\text { name }\end{array}$ & Type & Width & $\begin{array}{l}\text { Decimal } \\
\text { places }\end{array}$ & Description \\
\hline DATE_SAM & Date & 8 & & $\begin{array}{l}\text { Date the groundwater sample was taken } \\
\text { (key) }\end{array}$ \\
\hline DUP_Q & Logical & 1 & & $\begin{array}{l}\text { Whether it is a duplicate sample (key) } \\
\mathrm{T}=\text { True, it is a duplicate sample } \\
\mathrm{F}=\text { False, it is not a duplicate sample }\end{array}$ \\
\hline FILTER_Q & Logical & 1 & & $\begin{array}{l}\text { Whether it is a filtered sample (key) } \\
\begin{aligned} \mathrm{T} & =\text { True, it is a filtered sample } \\
\mathrm{F} & =\text { False, it is not a filtered sample }\end{aligned}\end{array}$ \\
\hline PCB_16 & Numeric & 16 & 4 & $\begin{array}{l}\text { Concentration of PCB (Aroclor 1016, } \\
\mu \mathrm{g} / \mathrm{L} \text { ) }\end{array}$ \\
\hline PCB_15_C & Character & 4 & & $\begin{array}{l}\text { Laboratory qualifiers for the } \\
\text { concentration of PCB (Aroclor 1016) }\end{array}$ \\
\hline PCB_21 & Numeric & 16 & 4 & $\begin{array}{l}\text { Concentration of PCB (Aroclor 1221, } \\
\mu \mathrm{g} / \mathrm{L} \text { ) }\end{array}$ \\
\hline PCB_21_C & Character & 4 & & $\begin{array}{l}\text { Laboratory qualifiers for the } \\
\text { concentration of PCB (Aroclor 1221) }\end{array}$ \\
\hline PCB_32 & Numeric & 16 & 4 & $\begin{array}{l}\text { Concentration of PCB (Aroclor 1232, } \\
\mu \mathrm{g} / \mathrm{L} \text { ) }\end{array}$ \\
\hline PCB_32_C & Character & 4 & & $\begin{array}{l}\text { Laboratory qualifiers for the } \\
\text { concentration of PCB (Aroclor 1232) }\end{array}$ \\
\hline PCB_42 & Numeric & 16 & 4 & $\begin{array}{l}\text { Concentration of PCB (Aroclor 1242, } \\
\mu \mathrm{g} / \mathrm{L} \text { ) }\end{array}$ \\
\hline PCB_42_C & Character & 4 & & $\begin{array}{l}\text { Laboratory qualifiers for the } \\
\text { concentration of PCB (Aroclor 1242) }\end{array}$ \\
\hline PCB_48 & Numeric & 16 & 4 & $\begin{array}{l}\text { Concentration of PCB (Aroclor 1248, } \\
\mu \mathrm{g} / \mathrm{L} \text { ) }\end{array}$ \\
\hline PCB_48_C & Character & 4 & & $\begin{array}{l}\text { Laboratory qualifiers for the } \\
\text { concentration of PCB (Aroclor 1248) }\end{array}$ \\
\hline
\end{tabular}


Table 5.8 (continued)

Variable

name

Type

Decimal

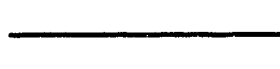

\begin{tabular}{|c|c|c|c|c|}
\hline PCB_54 & Numeric & 16 & 4 & $\begin{array}{l}\text { Concentration of PCB (Aroclor 1254, } \\
\mu \mathrm{g} / \mathrm{L} \text { ) }\end{array}$ \\
\hline PCB_54_C & Character & 4 & & $\begin{array}{l}\text { Laboratory qualifiers for the } \\
\text { concentration of PCB (Aroclor 1254) }\end{array}$ \\
\hline PCB_60 & Numeric & 16 & 4 & $\begin{array}{l}\text { Concentration of PCB (Aroclor 1260, } \\
\mu \mathrm{g} / \mathrm{L} \text { ) }\end{array}$ \\
\hline PCB_60_C & Character & 4 & & $\begin{array}{l}\text { Laboratory qualifiers for the } \\
\text { concentration of PCB (Aroclor 1260) }\end{array}$ \\
\hline PLANT & Character & 3 & & $\begin{array}{l}\text { Plant that installed the well (key) } \\
\begin{aligned} \mathrm{K} 25 & =\mathrm{K}-25 \\
\mathrm{X} 10 & =\mathrm{ORNL} \\
\mathrm{Y} 12 & =\mathrm{Y}-12\end{aligned}\end{array}$ \\
\hline SRC_PCB & Character & 30 & & $\begin{array}{l}\text { Source of the data } \\
\text { The plant the data came from is listed } \\
\text { first } \\
\text { K25 = K-25 } \\
\text { X10 = ORNL } \\
\text { Y12 = Y-12 } \\
\text { The type of the original data file is listed } \\
\text { second } \\
\text { CHEM = a groundwater analyses file } \\
\text { WELL = a well construction file } \\
\text { The original data file name is listed last, } \\
\text { in parentheses }\end{array}$ \\
\hline WELL & Character & 20 & & Official well name (key) \\
\hline
\end{tabular}


Table 5.9. Description of PEST file

\begin{tabular}{|c|c|c|c|c|}
\hline $\begin{array}{l}\text { Variable } \\
\text { name }\end{array}$ & Type & Width & $\begin{array}{l}\text { Decimal } \\
\text { places }\end{array}$ & Description \\
\hline ALDRNN & Numeric & 16 & 4 & Concentration of Aldrin $(\mu \mathrm{g} / \mathrm{L})$ \\
\hline ALDRIN_C & Character & 4 & & $\begin{array}{l}\text { Laboratory qualifiers for the } \\
\text { concentration of Aldrin }\end{array}$ \\
\hline BHC_A & Numeric & 16 & 4 & Concentration of $\alpha-\mathrm{BHC}(\mu \mathrm{g} / \mathrm{L})$ \\
\hline BHC_A_C & Character & 4 & & $\begin{array}{l}\text { Laboratory qualifiers for the } \\
\text { concentration of } \alpha-B H C\end{array}$ \\
\hline BHC_B & Numeric & 16 & 4 & Concentration of $\beta-\mathrm{BHC}(\mu \mathrm{g} / \mathrm{L})$ \\
\hline BHC_B_C & Character & 4 & & $\begin{array}{l}\text { Laboratory qualifiers for the } \\
\text { concentration of } \beta-B H C\end{array}$ \\
\hline BHC_D & Numeric & 16 & 4 & Concentration of $\delta$-BHC $(\mu \mathrm{g} / \mathrm{L})$ \\
\hline BHC_D_C & Character & 4 & & $\begin{array}{l}\text { Laboratory qualifiers for the } \\
\text { concentration of } \delta-B H C\end{array}$ \\
\hline BHC_G & Numeric & 16 & 4 & Concentration of $\gamma$-BHC $(\mu \mathrm{g} / \mathrm{L})$ \\
\hline BHC_G_C & Character & 4 & & $\begin{array}{l}\text { Laboratory qualifiers for the } \\
\text { concentration of } \gamma-\mathrm{BHC}\end{array}$ \\
\hline CLRD & Numeric & 16 & 4 & Concentration of Chlordane $(\mu \mathrm{g} / \mathrm{L})$ \\
\hline CLRD_C & Character & 4 & & $\begin{array}{l}\text { Laboratory qualifiers for the } \\
\text { concentration of Chlordane }\end{array}$ \\
\hline CLRD_A & Numeric & 16 & 4 & Concentration of $\alpha$-Chlordane $(\mu g / L)$ \\
\hline CLRD_A_C & Character & 4 & & $\begin{array}{l}\text { Laboratory qualifiers for the } \\
\text { concentration of } \alpha \text {-Chlordane }\end{array}$ \\
\hline CLRD_G & Numeric & 16 & 4 & Concentration of $\gamma$-Chlordane $(\mu \mathrm{g} / \mathrm{L})$ \\
\hline CLRD_G_C & Character & 4 & & $\begin{array}{l}\text { Laboratory qualifiers for the } \\
\text { concentration of } \gamma \text {-Chlordane }\end{array}$ \\
\hline
\end{tabular}


Table 5.9 (continued)

\begin{tabular}{|c|c|c|c|c|}
\hline $\begin{array}{l}\text { Variable } \\
\text { name }\end{array}$ & Type & Width & $\begin{array}{l}\text { Decimal } \\
\text { places }\end{array}$ & Description \\
\hline DATE_SAM & Date & 8 & & $\begin{array}{l}\text { Date the groundwater sample was taken } \\
\text { (key) }\end{array}$ \\
\hline DDD_44 & Numeric & 16 & 4 & Concentration of $4,4^{\prime}-\mathrm{DDD}(\mu \mathrm{J} / \mathrm{L})$ \\
\hline DDD_44_C & Character & 4 & & $\begin{array}{l}\text { Laboratory qualifiers for the } \\
\text { concentration of } 4,4^{\prime}-\mathrm{DDD}\end{array}$ \\
\hline DDE_44 & Numeric & 16 & 4 & Concentration of $4,4^{\prime}-\mathrm{DDE}(\mu \mathrm{g} / \mathrm{L})$ \\
\hline DDE_44_C & Character & 4 & & $\begin{array}{l}\text { Laboratory qualifiers for the } \\
\text { concentration of } 4,4^{\prime}-\mathrm{DDE}\end{array}$ \\
\hline DDT_44 & Numeric & 16 & 4 & Concentration of $4,4^{\prime}-\mathrm{DDT}(\mu \mathrm{g} / \mathrm{L})$ \\
\hline DDT_44_C & Character & 4 & & $\begin{array}{l}\text { Laboratory qualifiers for the } \\
\text { concentration of } 4,4^{\prime}-\text { DDT }\end{array}$ \\
\hline DIELDR & Numeric & 16 & 4 & Concentration of Dieldrin $(\mu \mathrm{g} / \mathrm{L})$ \\
\hline DIELDR_C & Character & 4 & & $\begin{array}{l}\text { Laboratory qualifiers for the } \\
\text { concentration of Dieldrin }\end{array}$ \\
\hline DUP_Q & Logical & 1 & & $\begin{array}{l}\text { Whether it is a duplicate sample (key) } \\
\qquad \begin{array}{l}T=\text { True, it is a duplicate sample } \\
\mathbf{F}=\text { False, it is not a duplicate sample }\end{array}\end{array}$ \\
\hline END & Numeric & 16 & 4 & Concentration of Endrin $(\mu \mathrm{g} / \mathrm{L})$ \\
\hline END_C & Character & 4 & & $\begin{array}{l}\text { Laboratory qualifiers for the } \\
\text { concentration of Endrin }\end{array}$ \\
\hline ENDALD & Numeric & 16 & 4 & Concentration of Endrin aldehyde $(\mu \mathrm{g} / \mathrm{L})$ \\
\hline ENDALD_C & Character & 4 & & $\begin{array}{l}\text { Laboratory qualifiers for the } \\
\text { concentration of Endrin aldehyde }\end{array}$ \\
\hline ENDKET & Numeric & 16 & 4 & Concentration of Endrin ketone $(\mu \mathrm{g} / \mathrm{L})$ \\
\hline
\end{tabular}


Table 5.9 (continued)

Variable

Decimal

name

Type

Width places

Description

\begin{tabular}{|c|c|c|c|c|}
\hline ENDKET_C & Character & 4 & & $\begin{array}{l}\text { Laboratory qualifiers for the } \\
\text { concentration of Endrin ketone }\end{array}$ \\
\hline ENSU_A & Numeric & 16 & 4 & Concentration of $\alpha$-Endosulfan $(\mu \mathrm{g} / \mathrm{L})$ \\
\hline ENSU_A_C & Character & 4 & & $\begin{array}{l}\text { Laboratory qualifiers for the } \\
\text { concentration of } \alpha \text {-Endosulfan }\end{array}$ \\
\hline ENSU_B & Numeric & 16 & 4 & Concentration of $\beta$-Endosulfan $(\mu \mathrm{g} / \mathrm{L})$ \\
\hline ENSU_B_C & Character & 4 & & $\begin{array}{l}\text { Laboratory qualifiers for the } \\
\text { concentration of } \beta \text {-Endosulfan }\end{array}$ \\
\hline ENSULS & Numeric & 16 & 4 & $\begin{array}{l}\text { Concentration of Endosulfan sulfate } \\
(\mu \mathrm{g} / \mathrm{L})\end{array}$ \\
\hline ENSULS_C & Character & 4 & & $\begin{array}{l}\text { Laboratory qualifiers for the } \\
\text { concentration of Endosulfan sulfate }\end{array}$ \\
\hline FILTER_Q & Logical & 1 & & $\begin{array}{l}\text { Whether it is a filtered sample (key) } \\
\begin{aligned} T & =\text { True, it is a filtered sample } \\
\mathrm{F} & =\text { False, it is not a filtered sample }\end{aligned}\end{array}$ \\
\hline HEPTCH & Numeric & 16 & 4 & Concentration of Heptachlor $(\mu \mathrm{g} / \mathrm{L})$ \\
\hline HEPTCH_C & Character & 4 & & $\begin{array}{l}\text { Laboratory qualifiers for the } \\
\text { concentration of Heptachlor }\end{array}$ \\
\hline HEPTEP & Numeric & 16 & 4 & $\begin{array}{l}\text { Concentration of Heptachlor epoxide } \\
(\mu \mathrm{g} / \mathrm{L})\end{array}$ \\
\hline HEPTEP_C & Character & 4 & & $\begin{array}{l}\text { Laboratory qualifiers for the } \\
\text { concentration of Heptachlor epoxide }\end{array}$ \\
\hline METHOX & Numeric & 16 & 4 & Concentration of Methoxychlor $(\mu \mathrm{g} / \mathrm{L})$ \\
\hline METHOX_C & Character & 4 & & $\begin{array}{l}\text { Laboratory qualifiers for the } \\
\text { concentration of Methoxychlor }\end{array}$ \\
\hline
\end{tabular}


Table 5.9 (continued)

Variable

name

Type

Decimal

Width places

Description

\begin{tabular}{|c|c|c|c|c|}
\hline PLANT & Character & 3 & & $\begin{array}{l}\text { Plant that installed the well (key) } \\
\begin{aligned} \mathrm{K} 25 & =\mathrm{K}-25 \\
\mathrm{X} 10 & =\mathrm{ORNL} \\
\mathrm{Y} 12 & =\mathrm{Y}-12\end{aligned}\end{array}$ \\
\hline SLVEX & Numeric & 16 & 4 & Concentration of Silvex $(\mu \mathrm{g} / \mathrm{L})$ \\
\hline SILVEX_C & Character & 4 & & $\begin{array}{l}\text { Laboratory qualifiers for the } \\
\text { concentration of Silvex }\end{array}$ \\
\hline SRC_PEST & Character & 30 & & $\begin{array}{l}\text { Source of the data } \\
\text { The plant the data came from is listed } \\
\text { first } \\
\text { K25 }=\text { K-25 } \\
\text { X10 }=\text { ORNL } \\
\text { Y12 = Y-12 } \\
\text { The type of the original data file is listed } \\
\text { second } \\
\text { CHEM = a groundwater analyses file } \\
\text { WELL = a well construction file } \\
\text { The original data file name is listed last, } \\
\text { in parentheses }\end{array}$ \\
\hline TOXAPH & Numeric & 16 & 4 & Concentration of Toxaphene $(\mu \mathrm{g} / \mathrm{L})$ \\
\hline TOXAPH_C & Character & 4 & & $\begin{array}{l}\text { Laboratory qualifiers for the } \\
\text { concentration of Toxaphene }\end{array}$ \\
\hline WELL & Character & 20 & & Official well name (key) \\
\hline
\end{tabular}


Table 5.10. Description of RAD file

Variable name

Type

Decimal

Width places Description

\begin{tabular}{|c|c|c|c|c|}
\hline ACT_A & Numeric & 16 & 4 & Gross alpha activity (pCi/L) \\
\hline ACT_A_C & Character & 4 & & $\begin{array}{l}\text { Laboratory qualifiers for the gross alpha } \\
\text { activity }\end{array}$ \\
\hline ACT_A_E & Numeric & 16 & 4 & Counting error for gross alpha activity \\
\hline ACT_B & Numeric & 16 & 4 & Gross beta activity (pCi/L) \\
\hline ACT_B_C & Character & 4 & & $\begin{array}{l}\text { Laboratory qualifiers for the gross beta } \\
\text { activity }\end{array}$ \\
\hline
\end{tabular}

ACT_B_E $\quad$ Numeric $16 \quad 4 \quad$ Counting error for gross beta activity

ACT_G Numeric $16 \quad 4 \quad$ Gross gamma activity (pCi/L)

ACT_G_C Character 4 Laboratory qualifiers for the gross gamma activity

ACT_G_E Numeric $16 \quad 4 \quad$ Counting error for gross gamma activity

AM_241 Numeric $16 \quad 4 \quad$ Concentration of americium-241 (pCi/L)

AM_241_C Character 4 Laboratory qualifiers for the

concentration of americium-241

AM_241_E Numeric $16 \quad 4 \quad$ Counting error for americium-241

ANT125 Numeric $16 \quad 4 \quad$ Concentration of antimony-125 (pCi/L)

ANT125_C Character 4 Laboratory qualifiers for the concentration of antimony-125

\begin{tabular}{|c|c|c|c|c|}
\hline ANT125_E & Numeric & 16 & 4 & Counting error for antimony-125 \\
\hline CER144 & Numeric & 16 & 4 & Concentration of cerium-144 ( $\mathrm{pCi} / \mathrm{L})$ \\
\hline CER144_C & Character & 4 & & $\begin{array}{l}\text { Laboratory qualifiers for the } \\
\text { concentration of cerium- } 144\end{array}$ \\
\hline
\end{tabular}


Table 5.10 (continued)

Variable name

Type

Decimal

Width places Description

\begin{tabular}{|c|c|c|c|c|}
\hline CER144_E & Numeric & 16 & 4 & Counting error for cerium-144 \\
\hline CES134 & Numeric & 16 & 4 & Concentration of cesium-134 (pCi/L) \\
\hline CES134_C & Character & 4 & & $\begin{array}{l}\text { Laboratory qualifiers for the } \\
\text { concentration of cesium-134 }\end{array}$ \\
\hline CES134_E & Numeric & 16 & 4 & Counting error for cesium-134 \\
\hline CES137 & Numeric & 16 & 4 & Concentration of cesium-137 (pCi/L) \\
\hline CES137_C & Character & 4 & & $\begin{array}{l}\text { Laboratory qualifiers for the } \\
\text { concentration of cesium- } 137\end{array}$ \\
\hline CES137_E & Numeric & 16 & 4 & Counting error for cesium-137 \\
\hline COBL60 & Numeric & 16 & 4 & Concentration of cobalt-60 (pCi/L) \\
\hline COBL60_C & Character & 4 & & $\begin{array}{l}\text { Laboratory qualifiers for the } \\
\text { concentration of cobalt }-60\end{array}$ \\
\hline COBL60_E & Numeric & 16 & 4 & Counting error for cobalt -60 \\
\hline DATE_SAM & Date & 8 & & $\begin{array}{l}\text { Date the groundwater sample was taken } \\
\text { (key) }\end{array}$ \\
\hline DUP_Q & Logical & 1 & & $\begin{array}{l}\text { Whether it is a duplicate sample (key) } \\
\qquad \begin{array}{l}\mathrm{T}=\text { True, it is a duplicaie sample } \\
\mathrm{F}=\text { False, it is not a duplicate sample }\end{array}\end{array}$ \\
\hline FILTER_Q & Logical & 1 & & $\begin{array}{l}\text { Whether it is a filtered sample (key) } \\
\qquad \begin{array}{l}T=\text { True, it is a filtered sample } \\
\mathbf{F}=\text { False, it is not a filtered sample }\end{array}\end{array}$ \\
\hline I_1259 & Numeric & 16 & 4 & $\begin{array}{l}\text { Concentration of iodine-125 and iodine- } \\
129(\mathrm{pCi} / \mathrm{L})\end{array}$ \\
\hline I_1259_C & Character & 4 & & $\begin{array}{l}\text { Laboratory qualifiers for the } \\
\text { concentration of iodine- } 125 \text { and } \\
\text { iodine- } 129\end{array}$ \\
\hline
\end{tabular}


Table 5.10 (continued)

Variable name
Decimal

Width places Description

I_1259_E Numeric $16 \quad 4 \quad$ Counting error for iodine-125 and iodine-129

I_131 Numeric 1644 Concentration of iodine-131 (pCi/L)

I_131_C Character 4 Laboratory qualifiers for the concentration of iodine-131

I_131_E Numeric $16 \quad 4 \quad$ Counting error for iodine-131

NEP237 Numeric $16 \quad 4 \quad$ Concentration of neptunium-237 (pCi/L)

NEP237_C Character 4 Laboratory qualifiers for the concentration of neptunium-237

NEP237_E Numeric $16 \quad 4 \quad$ Counting error for neptunium-237

NIOB95 Numeric $1644 \quad$ Concentration of niobium-95 (pCi/L)

NIOB95_C Character 4 Laboratory qualifiers for the concentration of niobium-95

NIOB95_E Numeric $16 \quad 4 \quad$ Counting error for niobium-95

$\begin{array}{lllll}\text { P_238 } & \text { Numeric } & 16 & 4 & \text { Concentration of plutonium-238 }(\mathrm{pCi} / \mathrm{L})\end{array}$

P_238_C Character 4 Laboratory qualifiers for the concentration of plutonium-238

P_238_E $\quad$ Numeric $16 \quad 4 \quad$ Counting error for plutonium-238

$\begin{array}{lllll}\text { P_239 Numeric } & 16 & 4 & \text { Concentration of plutonium-239 }(\mathrm{pCi} / \mathrm{L})\end{array}$

P_239_C Character 4 Laboratory qualifiers for the concentration of plutonium-239

P_239_E $\quad$ Numeric $16 \quad 4 \quad$ Counting error for plutonium-239

P_2394 Numeric $16 \quad 4 \quad$ Concentration of plutonium-239 and plutonium-240 (pCi/L) 
Table 5.10 (continued)

Variable name

Type

Decimal

Width places Description

P_2394_C Character 4

P_2394_E Numeric $16 \quad 4$

PLANT

P

PROTAC_C Character 4

PROTAC_E Numeric 16

RAD

RAD_C

Numeric 16

Character 4

RAD_E

Numeric

16

Numeric 16

RAD226_C Character 4

RAD226_E Numeric $16 \quad 4$

$\begin{array}{llll}\text { RAD228 } & \text { Numeric } & 16 & 4\end{array}$

RAD228_C Character 4

RAD228_E Numeric 16
Laboratory qualifiers for the concentration of plutonium-239 and plutonium-240

Counting error for plutonium-239 and plutonium-240

Plant that installed the well (key)

$$
\begin{aligned}
& \mathrm{K} 25=\mathrm{K}-25 \\
& \mathrm{X} 10=\mathrm{ORNL} \\
& \mathrm{Y} 12=\mathrm{Y}-12
\end{aligned}
$$

Concentration of protactinium $(\mathrm{pCi} / \mathrm{L})$

Laboratory qualifiers for the concentration of protactinium

Counting error for protactinium

Concentration of radium ( $\mathrm{pCi} / \mathrm{L}$ )

Laboratory qualifiers for the concentration of radium

Counting error for radium

Concentration of radium-226 (pCi/L)

Laboratory qualifiers for the concentration of radium- 226

Counting error for radium-226

Concentration of radium-228 (pCi/L)

Laboratory qualifiers for the concentration of radium-228

Counting error for radium-228 
Table 5.10 (continued)

\begin{tabular}{|c|c|c|c|c|}
\hline $\begin{array}{l}\text { Variable } \\
\text { name }\end{array}$ & Type & Width & $\begin{array}{l}\text { Decimal } \\
\text { places }\end{array}$ & Description \\
\hline RUT106 & Numeric & 16 & 4 & Concentration of ruthenium-106 (pCi/L) \\
\hline RUT106_C & Character & 4 & & $\begin{array}{l}\text { Laboratory qualifiers for the } \\
\text { concentration of ruthenium-106 }\end{array}$ \\
\hline RUT106_E & Numeric & 16 & 4 & Counting error for ruthenium-106 \\
\hline SRC_RAD & Character & 30 & & $\begin{array}{l}\text { Source of the data } \\
\text { The plant the data came from is listed } \\
\text { first } \\
\text { K25 }=\text { K-25 } \\
\text { X10 }=\text { ORNL } \\
\text { Y12 = Y-12 } \\
\text { The type of the original data file is listed } \\
\text { second } \\
\text { CHEM = a groundwater analyses file } \\
\text { WELL = a well construction file } \\
\text { The original data file name is listed last, } \\
\text { in parentheses }\end{array}$ \\
\hline STRN90 & Numeric & 16 & 4 & Concentration of strontium-90 (pCi/L) \\
\hline STRN90_C & Character & 4 & & $\begin{array}{l}\text { Laboratory qualifiers for the } \\
\text { concentration of strontium-90 }\end{array}$ \\
\hline STRN90_E & Numeric & 16 & 4 & Counting error for strontium-90 \\
\hline STRONT & Numeric & 16 & 4 & Concentration of strontium $(\mathrm{pCi} / \mathrm{L})$ \\
\hline STRONT_C & Character & 4 & & $\begin{array}{l}\text { Laboratory qualifiers for the } \\
\text { concentration of strontium }\end{array}$ \\
\hline STRONT_E & Numeric & 16 & 4 & Counting error for strontium \\
\hline TEC_99 & Numeric & 16 & 4 & Concentration of technetium- $99(\mathrm{pCi} / \mathrm{L})$ \\
\hline TEC_99_C & Character & 4 & & $\begin{array}{l}\text { Laboratory qualifiers for the } \\
\text { concentration of technetium-99 }\end{array}$ \\
\hline TEC_99_E & Numeric & 16 & 4 & Counting error for technetium-99 \\
\hline
\end{tabular}


Table 5.10 (continued)

Variable name

Type

Decimal

Width places

Description

\begin{tabular}{|c|c|c|c|c|}
\hline THA_28 & Numeric & 16 & 4 & Concentration of thallium-228 $(\mathrm{pCi} / \mathrm{L})$ \\
\hline THA_28_C & Character & 4 & & $\begin{array}{l}\text { Laboratory qualifiers for the } \\
\text { concentration of thallium-228 }\end{array}$ \\
\hline THA_28_E & Numeric & 16 & 4 & Counting error for thallium-228 \\
\hline THO_28 & Numeric & 16 & 4 & Concentration of thorium-228 $(\mathrm{pCi} / \mathrm{L})$ \\
\hline THO_28_C & Character & 4 & & $\begin{array}{l}\text { Laboratory qualifiers for the } \\
\text { concentration of thorium- } 228\end{array}$ \\
\hline THO_28_E & Numeric & 16 & 4 & Counting error for thorium-228 \\
\hline THO_30 & Numeric & 16 & 4 & Concentration of thorium-230 (pCi/L) \\
\hline THO_30_C & Character & 4 & & $\begin{array}{l}\text { Laboratory qualifiers for the } \\
\text { concentration of thorium- } 230\end{array}$ \\
\hline THO_30_E & Numeric & 16 & 4 & Counting error for thorium-230 \\
\hline THO_32 & Numeric & 16 & 4 & Concentration of thorium-232 (pCi/L) \\
\hline THO_32_C & Character & 4 & & $\begin{array}{l}\text { Laboratory qualifiers for the } \\
\text { concentration of thorium- } 232\end{array}$ \\
\hline THO_32_E & Numeric & 16 & 4 & Counting error for thorium-232 \\
\hline THO_34 & Numeric & 16 & 4 & Concentration of thorium-234 (pCi/L) \\
\hline THO_34_C & Character & 4 & & $\begin{array}{l}\text { Laboratory qualifiers for the } \\
\text { concentration of thorium- } 234\end{array}$ \\
\hline THO_34_E & Numeric & 16 & 4 & Counting error for thorium-234 \\
\hline THO314 & Numeric & 16 & 4 & $\begin{array}{l}\text { Concentration of thorium-231 and } \\
\text { thorium-234 (pCi/L) }\end{array}$ \\
\hline
\end{tabular}


Table 5.10 (continued)

\begin{tabular}{|c|c|c|c|c|}
\hline $\begin{array}{l}\text { Variable } \\
\text { name }\end{array}$ & Type & Width & $\begin{array}{l}\text { Decimal } \\
\text { places }\end{array}$ & Description \\
\hline THO314_C & Character & 4 & & $\begin{array}{l}\text { Laboratory qualifiers for the } \\
\text { concentration of thorium-231 and } \\
\text { thorium- } 234\end{array}$ \\
\hline THO314_E & Numeric & 16 & 4 & $\begin{array}{l}\text { Counting error for thorium-231 and } \\
\text { thorium-234 }\end{array}$ \\
\hline TRIT & Numeric & 16 & 4 & Concentration of tritium $(\mathrm{pCi} / \mathrm{L})$ \\
\hline TRIT_C & Character & 4 & & $\begin{array}{l}\text { Laboratory qualifiers for the } \\
\text { concentration of tritium }\end{array}$ \\
\hline TRIT_E & Numeric & 16 & 4 & Counting error for tritium \\
\hline$U_{-234}$ & Numeric & 16 & 4 & Concentration of uranium-234 (pCi/L) \\
\hline U_234_C & Character & 4 & & $\begin{array}{l}\text { Laboratory qualifiers for the } \\
\text { concentration of uranium- } 234\end{array}$ \\
\hline U_234_E & Numeric & 16 & 4 & Counting error for uranium-234 \\
\hline U_2356 & Numsric & 16 & 4 & $\begin{array}{l}\text { Concentration of uranium-235 and } \\
\text { uranium- } 236(\mathrm{pCi} / \mathrm{L})\end{array}$ \\
\hline U_2356_C & Character & 4 & & $\begin{array}{l}\text { Laboratory qualifiers for the } \\
\text { concentration of uranium- } 235 \text { and } \\
\text { uranium-236 }\end{array}$ \\
\hline U_2356_E & Numeric & 16 & 4 & $\begin{array}{l}\text { Counting error for uranium-235 and } \\
\text { uranium-236 }\end{array}$ \\
\hline U_238 & Numeric & 16 & 4 & Concentration of uranium-238 $(\mathrm{pCi} / \mathrm{L})$ \\
\hline U_238_C & Character & 4 & & $\begin{array}{l}\text { Laboratory qualifiers for the } \\
\text { concentration of uranium-238 }\end{array}$ \\
\hline U_238_E & Numeric & 16 & 4 & Counting error for uranium-238 \\
\hline WELL & Character & 20 & & Official well name (key) \\
\hline
\end{tabular}


Table 5.11. Description of VOC file

\begin{tabular}{|c|c|c|c|c|}
\hline $\begin{array}{l}\text { Variable } \\
\text { name }\end{array}$ & Type & Width & $\begin{array}{l}\text { Decimal } \\
\text { places }\end{array}$ & Description \\
\hline ACETON & Numeric & 16 & 4 & Concentration of acetone $(\mu \mathrm{g} / \mathrm{L})$ \\
\hline ACETON_C & Character & 4 & & $\begin{array}{l}\text { Laboratory qualifiers for the } \\
\text { concentration of acetone }\end{array}$ \\
\hline ACROLN & Numeric & 16 & 4 & Concentration of acrolein $(\mu \mathrm{g} / \mathrm{L})$ \\
\hline ACROLN_C & Character & 4 & & $\begin{array}{l}\text { Laboratory qualifiers for the } \\
\text { concentration of acrolein }\end{array}$ \\
\hline ACRYLT & Numeric & 16 & 4 & Concentration of acrylonitrile $(\mu \mathrm{g} / \mathrm{L})$ \\
\hline ACRYLT_C & Character & 4 & & $\begin{array}{l}\text { Laboratory qualifiers for the } \\
\text { concentration of acrylonitrile }\end{array}$ \\
\hline BENZEN & Numeric & 16 & 4 & Concentration of benzene $(\mu \mathrm{g} / \mathrm{L})$ \\
\hline BENZEN_C & Character & 4 & & $\begin{array}{l}\text { Laboratory qualifiers for the } \\
\text { concentration of benzene }\end{array}$ \\
\hline BROMID & Numeric & 16 & 4 & $\begin{array}{l}\text { Concentration of bromodichloromethane } \\
(\mu \mathrm{g} / \mathrm{L})\end{array}$ \\
\hline BROMID_C & Character & 4 & & $\begin{array}{l}\text { Laboratory qualifiers for the } \\
\text { concentration of bromodichloromethane }\end{array}$ \\
\hline BROMOF & Numeric & 16 & 4 & Concentration of bromoform $(\mu \mathrm{g} / \mathrm{L})$ \\
\hline BROMOF_C & Character & 4 & & $\begin{array}{l}\text { Laboratory qualifiers for the } \\
\text { concentration of bromoform }\end{array}$ \\
\hline BROMOM & Numeric & 16 & 4 & Concentration of bromomethane $(\mu \mathrm{g} / \mathrm{L})$ \\
\hline BROMOM_C & Character & 4 & & $\begin{array}{l}\text { Laboratory qualifiers for the } \\
\text { concentration of bromomethane }\end{array}$ \\
\hline BUTANO & Numeric & 16 & 4 & Concentration of 2-butanone $(\mu \mathrm{g} / \mathrm{L})$ \\
\hline BUTANO_C & Character & 4 & & $\begin{array}{l}\text { Laboratory qualifiers for the } \\
\text { concentration of 2-butanone }\end{array}$ \\
\hline
\end{tabular}


Table 5.11 (continued)

\begin{tabular}{|c|c|c|c|c|}
\hline $\begin{array}{l}\text { Variable } \\
\text { name }\end{array}$ & Type & Width & $\begin{array}{l}\text { Decimal } \\
\text { places }\end{array}$ & Description \\
\hline BUTENE & Numeric & 16 & 4 & Concentration of 1-butene $(\mu \mathrm{g} / \mathrm{L})$ \\
\hline BUTENE_C & Character & 4 & & $\begin{array}{l}\text { Laboratory qualifiers for the } \\
\text { concentration of 1-butene }\end{array}$ \\
\hline CARDIS & Numeric & 16 & 4 & Concentration of carbon disulfide $(\mu \mathrm{g} / \mathrm{L})$ \\
\hline CARDIS_C & Character & 4 & & $\begin{array}{l}\text { Laboratory qualifiers for the } \\
\text { concentration of carbon disulfide }\end{array}$ \\
\hline CARTET & Numeric & 16 & 4 & $\begin{array}{l}\text { Concentration of carbon tetrachloride } \\
(\mu \mathrm{g} / \mathrm{L})\end{array}$ \\
\hline CARTET_C & Characior & 4 & & $\begin{array}{l}\text { Laboratory qualifiers for the } \\
\text { concentration of carbon tetrachloride }\end{array}$ \\
\hline CHLBEN & Numeric & 16 & 4 & Concentration of chlorobenzene $(\mu \mathrm{g} / \mathrm{L})$ \\
\hline CHLBEN_C & Character & 4 & & $\begin{array}{l}\text { Laboratory qualifiers for the } \\
\text { concentration of chlorobenzene }\end{array}$ \\
\hline CHLDIB & Numeric & 16 & 4 & $\begin{array}{l}\text { Concentration of chlorodibromomethane } \\
\left(\mu \varepsilon^{\prime} / \mathrm{L}\right)\end{array}$ \\
\hline CHLDIB_C & Character & 4 & & $\begin{array}{l}\text { Laboratory qualifiers for the } \\
\text { concentration of chlorodibronuomethane }\end{array}$ \\
\hline CHLETH & Numeric & 16 & 4 & Concentration of chloroethane $(\mu \mathrm{g} / \mathrm{L})$ \\
\hline CHLETH_C & Character & 4 & & $\begin{array}{l}\text { Laboratory qualifiers for the } \\
\text { concentration of chloroethane }\end{array}$ \\
\hline CHLEVE & Pumeric & 16 & 4 & $\begin{array}{l}\text { Concentration of 2-chloroethyl vinyl } \\
\text { ether }(\mu \mathrm{g} / L)\end{array}$ \\
\hline CHLEVE_C & Characier & 4 & & $\begin{array}{l}\text { Labos atory qualifiers for the } \\
\text { concentration of } 2 \text {-chloroethyl vinyl ether }\end{array}$ \\
\hline CHLFOR & Numeric & 16 & 4 & Concentration of chloroform $(\mu \mathrm{g} / \mathrm{L})$ \\
\hline
\end{tabular}


Table 5.11 (continued)

\begin{tabular}{|c|c|c|c|c|}
\hline $\begin{array}{l}\text { Variable } \\
\text { name }\end{array}$ & Type & Width & $\begin{array}{l}\text { Decimal } \\
\text { places }\end{array}$ & Description \\
\hline CHLFOR_C & Character & 4 & & $\begin{array}{l}\text { Laboratory qualifiers for the } \\
\text { concentration of chloroform }\end{array}$ \\
\hline CHLMET & Numeric & 16 & 4 & Concentration of chloromethane $(\mu \mathrm{g} / \mathrm{L})$ \\
\hline CHLMET_C & Character & 4 & & $\begin{array}{l}\text { Laboratory qualifiers for the } \\
\text { concentration of chloromethane }\end{array}$ \\
\hline CHLTRI & Numeric & 16 & 4 & $\begin{array}{l}\text { Concentration of chlorotrifluoroethene } \\
(\mu \mathrm{g} / \mathrm{L})\end{array}$ \\
\hline CHLTRI_C & Character & 4 & & $\begin{array}{l}\text { Laboratory qualifiers for the } \\
\text { concentration of chlorotrifluoroethene }\end{array}$ \\
\hline CYCHEX & Numeric & 16 & 4 & Concentration of cyclohexane $(\mu \mathrm{g} / \mathrm{L})$ \\
\hline CYCHEX_C & Character & 4 & & $\begin{array}{l}\text { Laboratory qualifiers for the } \\
\text { concentration of cyclohexane }\end{array}$ \\
\hline DATE_SAM & Date & 8 & & $\begin{array}{l}\text { Date the groundwater sample was taken } \\
\text { (key) }\end{array}$ \\
\hline DCDIF & Numeric & 16 & 4 & $\begin{array}{l}\text { Concentration } \\
\text { dichlorodifluoromethane }(\mu \mathrm{g} / \mathrm{L})\end{array}$ \\
\hline DCDIF_C & Character & 4 & & $\begin{array}{l}\text { Laboratory qualifiers for the } \\
\text { concentration of dichlorodifluoromethane }\end{array}$ \\
\hline DCEA11 & Numeric & 16 & 4 & $\begin{array}{l}\text { Concentration of 1,1-dichloroethane } \\
(\mu \mathrm{g} / \mathrm{L})\end{array}$ \\
\hline DCEA11_C & Character & 4 & & $\begin{array}{l}\text { Laboratory qualifiers for the } \\
\text { concentration of 1,1-dichloroethane }\end{array}$ \\
\hline DCEA12 & Numeric & 16 & 4 & $\begin{array}{l}\text { Concentration of 1,2-dichloroethane } \\
(\mu \mathrm{g} / \mathrm{L})\end{array}$ \\
\hline DCEA12_C & Character & 4 & & $\begin{array}{l}\text { Laboratory qualifiers for the } \\
\text { concentration of } 1,2 \text {-dichloroethane }\end{array}$ \\
\hline
\end{tabular}


Table 5.11 (continued)

\begin{tabular}{|c|c|c|c|c|}
\hline $\begin{array}{l}\text { Variable } \\
\text { name }\end{array}$ & Type & Width & $\begin{array}{l}\text { Decimal } \\
\text { places }\end{array}$ & Description \\
\hline DCEE11 & Numeric & 16 & 4 & $\begin{array}{l}\text { Concentration of 1,1-dichloroethene } \\
(\mu \mathrm{g} / \mathrm{L})\end{array}$ \\
\hline DCEE11_C & Character & 4 & & $\begin{array}{l}\text { Laboratory qualifiers for the } \\
\text { concentration of } 1,1 \text {-dichloroethene }\end{array}$ \\
\hline DCEE12 & Numeric & 16 & 4 & $\begin{array}{l}\text { Concentration of 1,2-dichloroethene } \\
(\mu \mathrm{g} / \mathrm{L})\end{array}$ \\
\hline DCEE12_C & Character & 4 & & $\begin{array}{l}\text { Laboratory qualifiers for the } \\
\text { concentration of } 1,2 \text {-dichloroethene }\end{array}$ \\
\hline DCET12 & Numeric & 16 & 4 & $\begin{array}{l}\text { Concentration of trans-1,2-dichloroethene } \\
(\mu \mathrm{g} / \mathrm{L})\end{array}$ \\
\hline DCET12_C & Character & 4 & & $\begin{array}{l}\text { Laboratory qualifiers for the } \\
\text { concentration of trans-1,2-dichloroethene }\end{array}$ \\
\hline DCPC13 & Numeric & 16 & 4 & $\begin{array}{l}\text { Concentration of cis-1,3-dichloropropene } \\
(\mu \mathrm{g} / \mathrm{L})\end{array}$ \\
\hline DCPC13_C & Character & 4 & & $\begin{array}{l}\text { Laboratory qualifiers for the } \\
\text { concentration of cis-1,3-dichloropropene }\end{array}$ \\
\hline DCPT13 & Numeric & 16 & 4 & $\begin{array}{l}\text { Concentration of trans-1,3- } \\
\text { dichloropropene }(\mu \mathrm{g} / \mathrm{L})\end{array}$ \\
\hline DCPT13_C & Character & 4 & & $\begin{array}{l}\text { Laboratory qualifiers for the } \\
\text { concentration of } \\
\text { trans-1,3-dichloropropene }\end{array}$ \\
\hline DCP12 & Numeric & 16 & 4 & $\begin{array}{l}\text { Concentration of 1,2-dichloropropane } \\
(\mu \mathrm{g} / \mathrm{L})\end{array}$ \\
\hline DCP12_C & Character & 4 & & $\begin{array}{l}\text { Laboratory qualifiers for the } \\
\text { concentration of } 1,2 \text {-dichloropropane }\end{array}$ \\
\hline DCTET & Numeric & 16 & 4 & $\begin{array}{l}\text { Concentration of } 1,2 \text {-dichloro- } \\
1,1,2,2 \text {-tetrafluoroethane }(\mu \mathrm{g} / \mathrm{L})\end{array}$ \\
\hline
\end{tabular}


Table 5.11 (continued)

Variable name

Type

Decimal

Width places Description

\begin{tabular}{|c|c|c|c|c|}
\hline DCTET_C & Character & 4 & & $\begin{array}{l}\text { Laboratory qualifiers for the } \\
\text { concentration of } \\
\text { 1,2-dichloro-1,1,2,2-tetrafluoroethane }\end{array}$ \\
\hline DCTRI & Numeric & 16 & 4 & $\begin{array}{l}\text { Concentration of } \\
1,2 \text {-dichloro-1,1,2-trifluoroethane }(\mu \mathrm{g} / \mathrm{L})\end{array}$ \\
\hline DCTRI_C & Character & 4 & & $\begin{array}{l}\text { Laboratory qualifiers for the } \\
\text { concentration of } 1,2 \text {-dichloro-1,1,2- } \\
\text { trifluoroethane }\end{array}$ \\
\hline DMBT23 & Numeric & 16 & 4 & $\begin{array}{l}\text { Concentration of 2,3-dimethylbutane } \\
(\mu g / L)\end{array}$ \\
\hline DMBT23_C & Character & 4 & & $\begin{array}{l}\text { Laboratory qualifiers for the } \\
\text { concentration of 2,3-dimethylbutane }\end{array}$ \\
\hline DMPN24 & Numeric & 16 & 4 & $\begin{array}{l}\text { Concentration of 2,4-dimethylpentane } \\
(\mu \mathrm{g} / \mathrm{L})\end{array}$ \\
\hline DMPN24_C & Character & 4 & & $\begin{array}{l}\text { Laboratory qualifiers for the } \\
\text { concentration of } 2,4 \text {-dimethylpentane }\end{array}$ \\
\hline DUP_Q & Logical & 1 & & $\begin{array}{l}\text { Whether it is a duplicate sample (key) } \\
\qquad \begin{array}{l}\mathbf{T}=\text { True, it is a duplicate sample } \\
\mathbf{F}=\text { False, it is not a duplicate sample }\end{array}\end{array}$ \\
\hline ETBENZ & Numeric & 16 & 4 & Concentration of ethylbenzene $(\mu \mathrm{g} / \mathrm{L})$ \\
\hline ETBENZ_C & Character & 4 & & $\begin{array}{l}\text { Laboratory qualifiers for the } \\
\text { concentration of ethylbenzene }\end{array}$ \\
\hline ETCYCB & Numeric & 16 & 4 & Concentration of ethylcyclobutane $(\mu \mathrm{g} / \mathrm{L})$ \\
\hline ETCYCB_C & Character & 4 & & $\begin{array}{l}\text { Laboratory qualifiers for the } \\
\text { concentration of ethylcyclobutane }\end{array}$ \\
\hline ETMETB & Numeric & 16 & 4 & $\begin{array}{l}\text { Concentration of 1-ethyl-2-methyl- } \\
\text { benzene }(\mu \mathrm{g} / \mathrm{L})\end{array}$ \\
\hline
\end{tabular}


Table 5.11 (continued)

Variable name Type

Decimal

ETMETB_C Character 4

Width places

Description

\begin{tabular}{|c|c|c|c|c|}
\hline ETMETB_C & Character & 4 & & $\begin{array}{l}\text { Laboratory qualifiers for the } \\
\text { concentration of } 1 \text {-ethyl-2-methyl- } \\
\text { benzene }\end{array}$ \\
\hline FILTER_Q & Logical & 1 & & $\begin{array}{l}\text { Whether it is a filtered sample (key) } \\
\begin{array}{l}\mathrm{T}=\text { True, it is a filtered sample } \\
\mathrm{F}=\text { False, it is not a filtered sample }\end{array}\end{array}$ \\
\hline FLTRIC & Numeric & 16 & 4 & $\begin{array}{l}\text { Concentration of fluorotrichloromethane } \\
(\mu \mathrm{g} / \mathrm{L})\end{array}$ \\
\hline FLTRIC_C & Character & 4 & & $\begin{array}{l}\text { Laboratory qualifiers for the } \\
\text { concentration of fluorotrichloromethane }\end{array}$ \\
\hline HEXAMT & Numeric & 16 & 4 & $\begin{array}{l}\text { Concentration of } \\
\text { hexamethylcyclotrisiloxane }(\mu \mathrm{g} / \mathrm{L})\end{array}$ \\
\hline HEXAMT_C & Character & 4 & & $\begin{array}{l}\text { Laboratory qualifiers for the } \\
\text { concentration of } \\
\text { hexamethylcyclotrisiloxane }\end{array}$ \\
\hline HEXANO & Numeric & 16 & 4 & Concentration of 2-hexanone $(\mu \mathrm{g} / \mathrm{L})$ \\
\hline HEXANO_C & Character & 4 & & $\begin{array}{l}\text { Laboratory qualifiers for the } \\
\text { concentration of } 2 \text {-hexanone }\end{array}$ \\
\hline METOX & Numeric & 16 & 4 & $\begin{array}{l}\text { Concentration of } \\
\text { 2-methoxy-2-methylpropane }(\mu \mathrm{g} / \mathrm{L})\end{array}$ \\
\hline METOX_C & Character & 4 & & $\begin{array}{l}\text { Laboratory qualifiers for the concentration } \\
\text { of } 2 \text {-methoxy-2-methylpropane }\end{array}$ \\
\hline MTHBUT & Numeric & 16 & 4 & Concentration of 2-methyl-butane $(\mu \mathrm{g} / \mathrm{L})$ \\
\hline MTHBUT_C & Character & 4 & & $\begin{array}{l}\text { Laboratory qualifiers for the concentration } \\
\text { of } 2 \text {-methyl-butane }\end{array}$ \\
\hline MTHCYH & Numeric & 16 & 4 & Concentration of methylcyclohexane $(\mu \mathrm{g} / \mathrm{L})$ \\
\hline
\end{tabular}


Table 5.11 (continued)

\begin{tabular}{|c|c|c|c|c|}
\hline $\begin{array}{l}\text { Variable } \\
\text { name }\end{array}$ & Type & Width & $\begin{array}{l}\text { Decimal } \\
\text { places }\end{array}$ & Description \\
\hline MTHCYH_C & Character & 4 & & $\begin{array}{l}\text { Laboratory qualifiers for the } \\
\text { concentration of methylcyclohexane }\end{array}$ \\
\hline MTHCYP & Numeric & 16 & 4 & $\begin{array}{l}\text { Concentration of methylcyclopentane } \\
(\mu \mathrm{g} / \mathrm{L})\end{array}$ \\
\hline MTHCYP_C & Character & 4 & & $\begin{array}{l}\text { Laboratory qualifiers for the } \\
\text { concentration of methylcyclopentane }\end{array}$ \\
\hline MTHECL & Numeric & 16 & 4 & $\begin{array}{l}\text { Concentration of methylene chloride } \\
(\mu \mathrm{g} / \mathrm{L})\end{array}$ \\
\hline MTHECL_C & Character & 4 & & $\begin{array}{l}\text { Laboratory qualifiers for the } \\
\text { concentration of methylene chloride }\end{array}$ \\
\hline MTHP_2 & Numeric & 16 & 4 & Concentration of 2-methylpentane $(\mu \mathrm{g} / \mathrm{L})$ \\
\hline MTHP_2_C & Character & 4 & & $\begin{array}{l}\text { Laboratory qualifiers for the } \\
\text { concentration of } 2 \text {-methylpentane }\end{array}$ \\
\hline MTHP_3 & Numeric & 16 & 4 & Concentration of 3-methylpentane $(\mu \mathrm{g} / \mathrm{L})$ \\
\hline MTHP_3_C & Character & 4 & & $\begin{array}{l}\text { Laboratory qualifiers for the } \\
\text { concentration of 3-methylpentane }\end{array}$ \\
\hline MTHPEN & Numeric & 16 & 4 & $\begin{array}{l}\text { Concentration of 4-methyl-2-pentanone } \\
(\mu \mathrm{g} / \mathrm{L})\end{array}$ \\
\hline MTHPEN_C & Character & 4 & & $\begin{array}{l}\text { Laboratory qualifiers for the concentration } \\
\text { of 4-methyl-2-pentanone }\end{array}$ \\
\hline MTHPRO & Numeric & 16 & 4 & $\begin{array}{l}\text { Concentration of 2-methyl-1-propane } \\
(\mu \mathrm{g} / \mathrm{L})\end{array}$ \\
\hline MTHPRO_C & Character & 4 & & $\begin{array}{l}\text { Laboratory qualifiers for the concentration } \\
\text { of 2-methyl-1-propane }\end{array}$ \\
\hline
\end{tabular}


Table 5.11 (continued)

\begin{tabular}{|c|c|c|c|c|}
\hline $\begin{array}{l}\text { Variable } \\
\text { name }\end{array}$ & Type & Width & $\begin{array}{l}\text { Decimal } \\
\text { places }\end{array}$ & Description \\
\hline PLANT & Character & 3 & & $\begin{array}{l}\text { Plant that installed the well (key) } \\
\begin{aligned} \text { K25 } & =\mathrm{K}-25 \\
\mathrm{X} 10 & =\mathrm{ORNL} \\
\mathrm{Y} 12 & =\mathrm{Y}-12\end{aligned}\end{array}$ \\
\hline SRC_VOC & Character & 30 & & $\begin{array}{l}\text { Source of the data } \\
\text { The plant the data came from is listed } \\
\text { first } \\
\text { K25 = K-25 } \\
\text { X10 = ORNL } \\
\text { Y12 = Y-12 } \\
\text { The type of the original data file is listed } \\
\text { second } \\
\text { CHEM = a groundwater analyses file } \\
\text { WELL = a well construction file } \\
\text { The original data file name is listed last, } \\
\text { in parentheses }\end{array}$ \\
\hline STYREN & Numeric & 16 & 4 & Concentration of styrene $(\mu \mathrm{g} / \mathrm{L})$ \\
\hline STYREN_C & Character & 4 & & $\begin{array}{l}\text { Laboratory qualifiers for the } \\
\text { concentration of styrene }\end{array}$ \\
\hline TECETA & Numeric & 16 & 4 & $\begin{array}{l}\text { Concentration of } 1,1,2,2- \\
\text { tetrachloroethane }(\mu \mathrm{g} / \mathrm{L})\end{array}$ \\
\hline TECETA_C & Character & 4 & & $\begin{array}{l}\text { Laboratory qualifiers for the } \\
\text { concentration of } \\
\text { 1,1,2,2-tetrachloroethane }\end{array}$ \\
\hline TECETE & Numeric & 16 & 4 & $\begin{array}{l}\text { Concentration of tetrachloroethene } \\
(\mu \mathrm{g} / \mathrm{L})\end{array}$ \\
\hline TECETE_C & Character & 4 & & $\begin{array}{l}\text { Laboratory qualifiers for the } \\
\text { concentration of tetrachloroethene }\end{array}$ \\
\hline TETMTS & Numeric & 16 & 4 & Concentration of tetramethylsilane $(\mu \mathrm{g} / \mathrm{L})$ \\
\hline
\end{tabular}


Table 5.11 (continued)

\begin{tabular}{|c|c|c|c|c|}
\hline $\begin{array}{l}\text { Variable } \\
\text { name }\end{array}$ & Type & Width & $\begin{array}{l}\text { Decimal } \\
\text { places }\end{array}$ & Description \\
\hline TETMTS_C & Character & 4 & & $\begin{array}{l}\text { Laboratory qualifiers for the } \\
\text { concentration of tetramethylsilane }\end{array}$ \\
\hline TOLUEN & Numeric & 16 & 4 & Concentration of toluene $(\mu \mathrm{g} / \mathrm{L})$ \\
\hline TOLUEN_C & Character & 4 & & $\begin{array}{l}\text { Laboratory qualifiers for the } \\
\text { concentration of toluene }\end{array}$ \\
\hline TRC111 & Numeric & 16 & 4 & $\begin{array}{l}\text { Concentration of 1,1,1-trichloroethane } \\
(\mu \mathrm{g} / \mathrm{L})\end{array}$ \\
\hline TRC111_C & Character & 4 & & $\begin{array}{l}\text { Laboratory qualifiers for the concentration } \\
\text { of } 1,1,1 \text {-trichloroethane }\end{array}$ \\
\hline TRC112 & Numeric & 16 & 4 & $\begin{array}{l}\text { Concentration of 1,1,2-trichloroethane } \\
(\mu \mathrm{g} / \mathrm{L})\end{array}$ \\
\hline TRC112_C & Character & 4 & & $\begin{array}{l}\text { Laboratory qualifiers for the concentration } \\
\text { of } 1,1,2 \text {-trichloroethane }\end{array}$ \\
\hline TRICLE & Numeric & 16 & 4 & Concentration of trichloroethene $(\mu \mathrm{g} / \mathrm{L})$ \\
\hline TRICLE_C & Character & 4 & & $\begin{array}{l}\text { Laboratory qualifiers for the concentration } \\
\text { of trichloroethene }\end{array}$ \\
\hline TRICTR & Numeric & 16 & 4 & Concentration of trifluoroethene $(\mu \mathrm{g} / \mathrm{L})$ \\
\hline TRICTR_C & Character & 4 & & $\begin{array}{l}\text { Laboratory qualifiers for the concentration } \\
\text { of trifluoroethene }\end{array}$ \\
\hline TRIFLE & Numeric & 16 & 4 & $\begin{array}{l}\text { Concentration of } \\
\text { 1,1,2-trichloro-1,2,2-trifluoroethane } \\
(\mu \mathrm{g} / \mathrm{L})\end{array}$ \\
\hline TRIFLE_C & Character & 4 & & $\begin{array}{l}\text { Laboratory qualifiers for the concentration } \\
\text { of 1,1,2-trichloro-1,2,2-trifluoroethane }\end{array}$ \\
\hline TRM135 & Numeric & 16 & 4 & $\begin{array}{l}\text { Concentration of } 1,3,5 \text {-trimethylbenzene } \\
(\mu \mathrm{g} / \mathrm{L})\end{array}$ \\
\hline
\end{tabular}


Table 5.11 (continued)

Variable name

Type

Decimal

Width places Description

TRM135_C Character 4

Laboratory qualifiers for the concentration of 1,3,5-trimethylbenzene

VNYACE Numeric 16

Concentration of vinyl acetate $(\mu \mathrm{g} / \mathrm{L})$

VNYACE_C Character 4

Laboratory qualifiers for the concentration of vinyl acetate

VNYCHL Numeric 16

Concentration of vinyl chloride $(\mu \mathrm{g} / \mathrm{L})$

VNYCHL_C Character 4

Laboratory qualifiers for the concentration of vinyl chloride

WELL Character 20

Official well name (key)

XYLENE Numiric 16

4

Concentration of total xylenes $(\mu \mathrm{g} / \mathrm{L})$

XYLENE_C Character 4

Laboratory qualifiers for the concentration of total xylenes 
Table 5.12 Description of WCON file

Variable

Decimal

name

Type

Width places

Description

\begin{tabular}{|c|c|c|c|c|}
\hline CASBOT_1 & Numeric & 16 & 4 & $\begin{array}{l}\text { Depth from land surface to the bottom } \\
\text { of the first casing (ft) }\end{array}$ \\
\hline CASBOT_2 & Numeric & 16 & 4 & $\begin{array}{l}\text { Depth from land surface to the bottom } \\
\text { of the second casing (ft) }\end{array}$ \\
\hline CASBOT_3 & Numeric & 16 & 4 & $\begin{array}{l}\text { Depth from land surface to the bottom } \\
\text { of the third casing ( } f t)\end{array}$ \\
\hline CASDI_1 & Numeric & 16 & 4 & Diameter of the first casing (in.) \\
\hline CASDI_2 & Numeric & 16 & 4 & Diameter of the second casing (in.) \\
\hline CASDII_1 & Numeric & 16 & 4 & Inside diameter of the first casing (in.) \\
\hline CASDIO_1 & Numeric & 16 & 4 & Outside diameter of the first casing (in.) \\
\hline CASDIO_2 & Numeric & 16 & 4 & $\begin{array}{l}\text { Outside diameter of the second casing } \\
\text { (in.) }\end{array}$ \\
\hline CASDIO_3 & Numeric & 16 & 4 & Outside diameter of the third casing (in.) \\
\hline CASMAT_1 & Character & 15 & & First casing material \\
\hline CASMAT_2 & Character & 15 & & Second casing material \\
\hline CASMAT_3 & Character & 15 & & Third casing material \\
\hline CASTOP_1 & Numeric & 16 & 4 & $\begin{array}{l}\text { Elevation of the top of the first casing } \\
\text { (feet above mean sea level) }\end{array}$ \\
\hline COMCON_1 & Character & 80 & & Comment one for well construction \\
\hline COMCON_2 & Character & 80 & & Comment two for well construction \\
\hline COMCON_3 & Character & 80 & & Comment three for well construction \\
\hline COMCON_4 & Character & 80 & & Comment four for well construction \\
\hline COMCON & Character & 80 & & Comment five for well construction \\
\hline
\end{tabular}


Table 5.12 (continued)

\begin{tabular}{|c|c|c|c|c|}
\hline $\begin{array}{l}\text { Variable } \\
\text { name }\end{array}$ & Type & Width & $\begin{array}{l}\text { Decimal } \\
\text { places }\end{array}$ & Description \\
\hline & & & & $\therefore$ \\
\hline COMCON_6 & Character & 80 & & Comment six for well construction \\
\hline COMCON_? & Character & 80 & & Comment seven for well construction \\
\hline COMCON_8 & Character & 80 & & Comment eight for well construction \\
\hline COND_DEV & Numeric & 16 & 4 & $\begin{array}{l}\text { Specific conductance of water at the time } \\
\text { of development (us } / \mathrm{cm} \text { ) }\end{array}$ \\
\hline CONSMTHD & Character & 20 & & Method of well construction \\
\hline CONTNAME & Character & 25 & & Well contractor \\
\hline CSHBOT_1 & Numeric & 16 & 4 & $\begin{array}{l}\text { Depth from land surface to the bottom } \\
\text { of the first casing hole (ft) }\end{array}$ \\
\hline CSHBOT_2 & Numeric & 16 & 4 & $\begin{array}{l}\text { Depth from land surface to the bottom } \\
\text { of the second casing hole (ft) }\end{array}$ \\
\hline CSHDI_1 & Numeric & 16 & 4 & Diameter of the first casing hole (in.) \\
\hline CSHDI_2 & Numeric & 16 & 4 & Diameter of the second casing hole (in.) \\
\hline CSHDI_3 & Numeric & 16 & 4 & Diameter of the third casing hole (in.) \\
\hline DATE_CON & Date & 8 & & $\begin{array}{l}\text { Date a well was ccinpleted or the date } \\
\text { the bore was taken. Note: In some cases } \\
\text { the actual date is listed and in other } \\
\text { cases only the year is given }\end{array}$ \\
\hline DATE_DES & Date & 8 & & Date a well was destroyed \\
\hline MP & Numeric & 16 & 4 & Measure point elevation ( $f t$ ) \\
\hline MP_COR & Numeric & 16 & 4 & $\begin{array}{l}\text { Measure point correction, above }(-) \text { or } \\
\text { below }(+) \text { land surface }(\mathrm{ft})\end{array}$ \\
\hline OPNBOT_1 & Numeric & 16 & 4 & $\begin{array}{l}\text { Depth from land surface to the bottom } \\
\text { of the first open interval (ft) }\end{array}$ \\
\hline
\end{tabular}


Table 5.12 (continued)

Variable name
Type
Decimal

Width places Description
OPNBOT_2 Numeric $16 \quad 4$

OPNDI_1 Numeric $16 \quad 4$

OPNDI_2 $\quad$ Numeric $16 \quad 4$

OPNTOP_1 Numeric $16 \quad 4$

OPNTOP_2 Numeric $16 \quad 4$

$\begin{array}{llll}\text { PACKBOT Numeric } & 16 & 4\end{array}$

$\begin{array}{llll}\text { PACKTOP } & \text { Numeric } & 16 & 4\end{array}$

PLANT

Character 3

SCRBOT_1 Numeric $16 \quad 4$

SCRBOT_2 Numeric $16 \quad 4$

$\begin{array}{llll}\text { SCRBOT_3 Numeric } & 16 & 4\end{array}$

SCRBOT_4 Numeric $16 \quad 4$

SCRDI_1 Numeric $16 \quad 4$

SCRDII_1 Numeric $16 \quad 4$
Depth from land surface to the bottom of the second open interval ( $f t$ )

Diameter of the first open interval (in.)

Diameter of the second open interval (in.)

Depth from land surface to the top of the first open interval (ft)

Depth from land surface to the top of the second open interval (ft)

Depth from land surface to the bottom of the packed interval ( $f t$ )

Depth from land surface to the top of the packed interval ( $f t)$

Plant that installed the well (key)

$$
\begin{aligned}
& \mathrm{K} 25=\mathrm{K}-25 \\
& \mathrm{X} 10=\mathrm{ORNL} \\
& \mathrm{Y} 12=\mathrm{Y}-12
\end{aligned}
$$

Depth from land surface to the bottom of the first screened interval ( $f t)$

Depth from land surface to the bottom of the second screened interval ( $f t$ )

Depth from land surface to the bottom of the third screened interval $(f t)$

Depth from land surface to the bottom of the forth screened interval $(\mathrm{ft})$

Diameter of the screen (in.)

Inside diameter of the screen (in.) 
Table 5.12 (continued)

Variable name

Type

Width places

Description

\begin{tabular}{|c|c|c|c|c|}
\hline SCRMAT_1 & Character & 15 & & Material composition of the first screen \\
\hline SCRSLTSZ & Numeric & 16 & 4 & Screen slot size (in.) \\
\hline SCRTOP_1 & Numeric & 16 & 4 & $\begin{array}{l}\text { Depth from land surface to the top of } \\
\text { the first screen (ft) }\end{array}$ \\
\hline SCRTOP_2 & Numeric & 16 & 4 & $\begin{array}{l}\text { Depth from land surface to the top of } \\
\text { the second screen (ft) }\end{array}$ \\
\hline SCRTOP_3 & Numeric & 16 & 4 & $\begin{array}{l}\text { Depth from land surface to the top of } \\
\text { the third screen (ft) }\end{array}$ \\
\hline SCRTOP_4 & Numeric & 16 & 4 & $\begin{array}{l}\text { Depth from land surface to the top of } \\
\text { the forth screen (ft) }\end{array}$ \\
\hline SEALBOT & Numeric & 16 & 4 & Depth to the bottom of the seal ( $\mathrm{ft}$ ) \\
\hline SEALTOP & Numeric & 16 & 4 & $\begin{array}{l}\text { Depth from land surface to the top of } \\
\text { the seal (ft) }\end{array}$ \\
\hline SRC_WCON & Character & 30 & & $\begin{array}{l}\text { Source of the data } \\
\text { The plant the data came from is listed } \\
\text { first } \\
\text { K25 = K-25 } \\
\mathrm{X} 10=\mathrm{ORNL} \\
\mathrm{Y} 12=\mathrm{Y}-12 \\
\text { The type of the original data file is listed } \\
\text { second } \\
\text { CHEM = a groundwater analyses file } \\
\text { WELL = a well construction file } \\
\text { The original data file name is listed last, } \\
\text { in parentheses }\end{array}$ \\
\hline
\end{tabular}

$\begin{array}{llll}\text { WELDEP } & \text { Numeric } & 16 & 4\end{array}$

Total depth of the well (ft) below land surface The total depth value is the "as built" depth, based on drilling records or on well measurement immediately after construction 
Table 5.12 (continued)

\begin{tabular}{|c|c|c|c|c|}
\hline $\begin{array}{l}\text { Variable } \\
\text { name }\end{array}$ & Type & Width & $\begin{array}{l}\text { Decimal } \\
\text { places }\end{array}$ & Description \\
\hline WELL & Character & 20 & & Official well name (key) \\
\hline
\end{tabular}




\section{WELL DIAGRAMS}

\section{CONTENTS OF SECTION 6}

Fig. 6.1 Diagram of an open well with one casing ......... 81

Fig. 6.2 Diagram of an open well with two casings $\ldots \ldots \ldots \ldots 8$

Fig. 6.3 Diagram of an open well with three casings $\ldots \ldots \ldots \ldots 3$

Fig. 6.4 Diagram of a screened well with one casing ......... 84

Fig. 6.5 Diagram of a screened well with two casings $\ldots \ldots \ldots \ldots 85$

Fig. 6.6 Diagram of a screened well with three casings ........ 86 


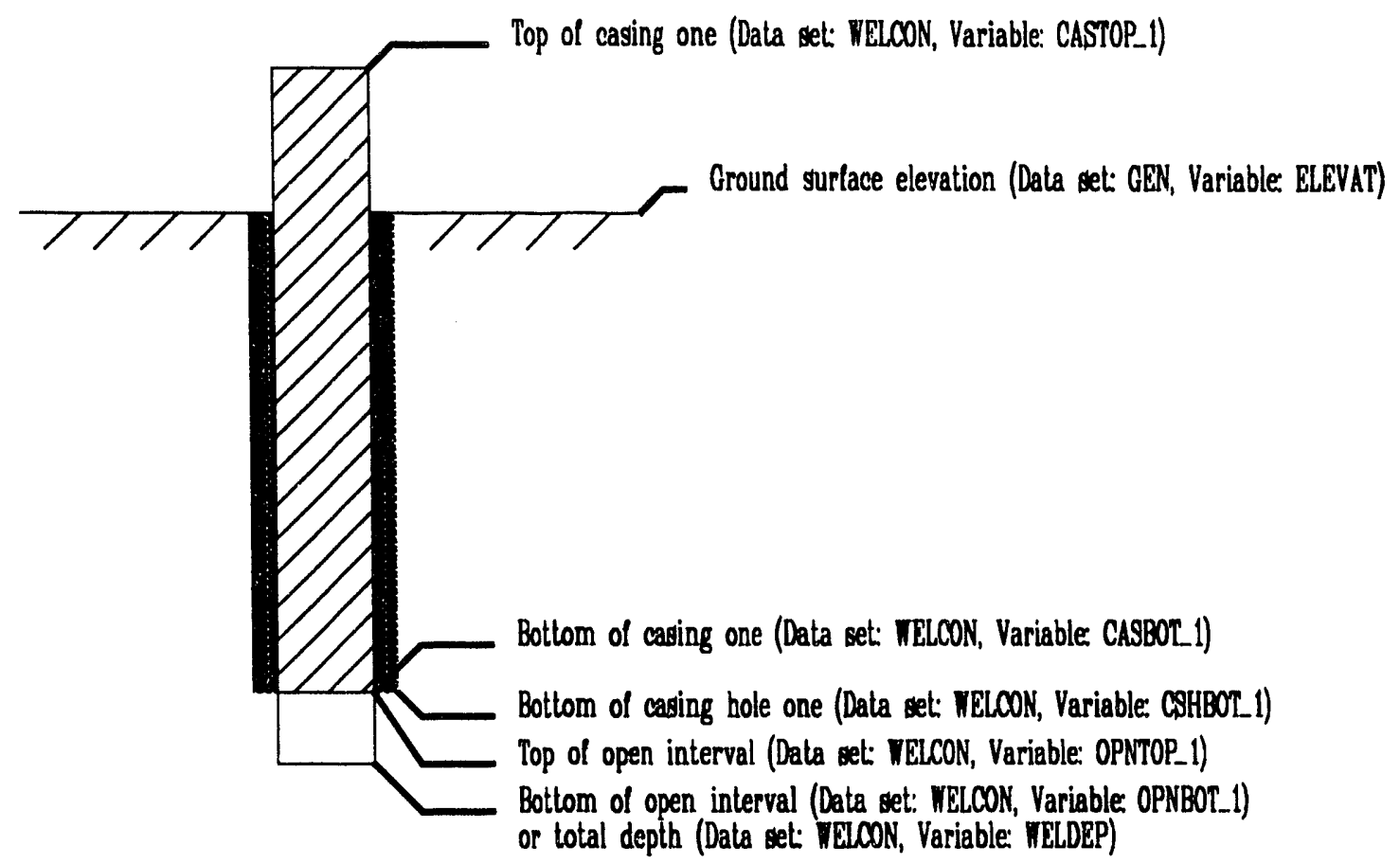

Key:

Grout

Z71 Casing one

6.1. Diagram of an open well with one casing. 


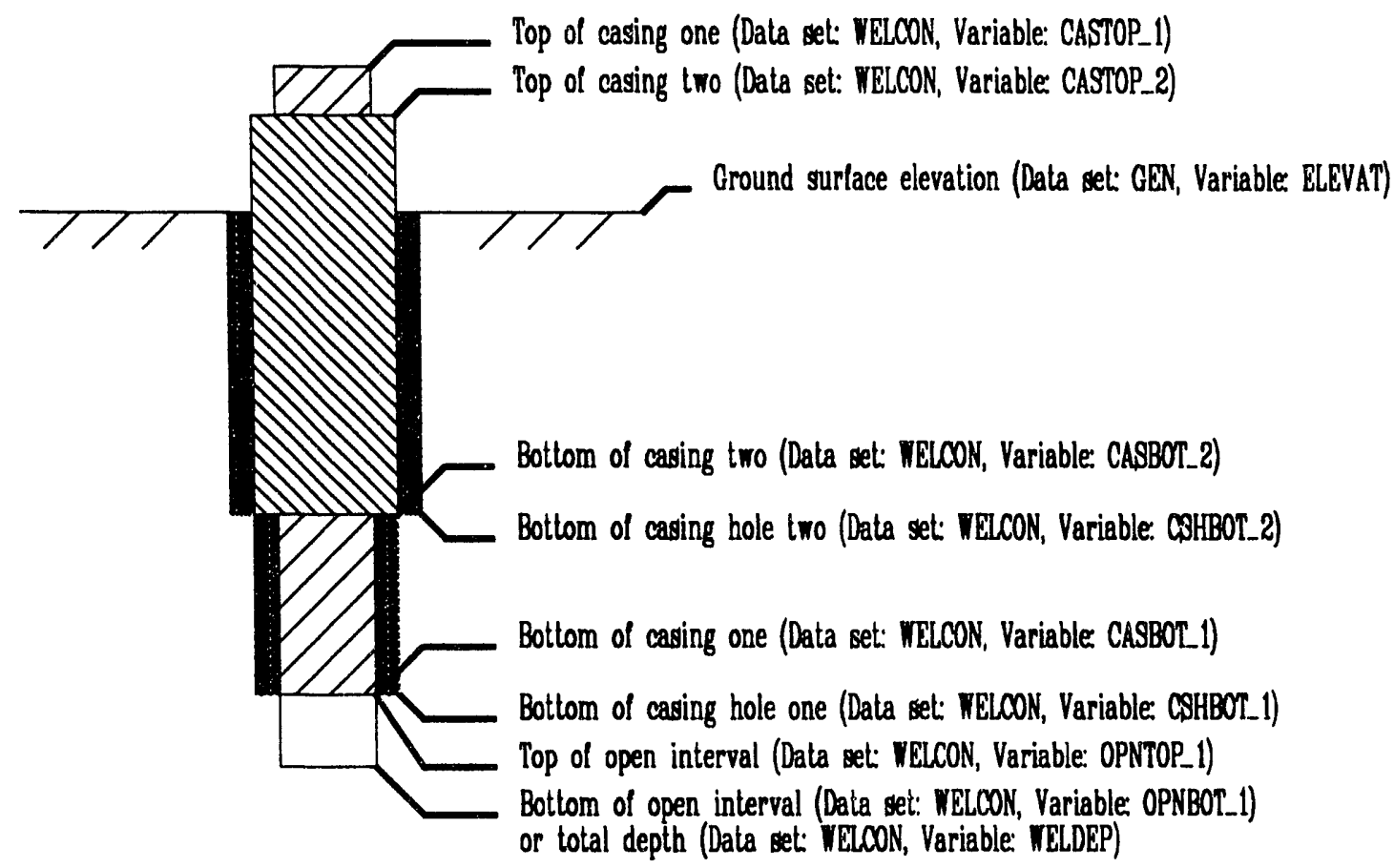

Key:

Grout

ZZA Casing one

MIII Casing tro

6.2. Diagram of an open well with two casings. 


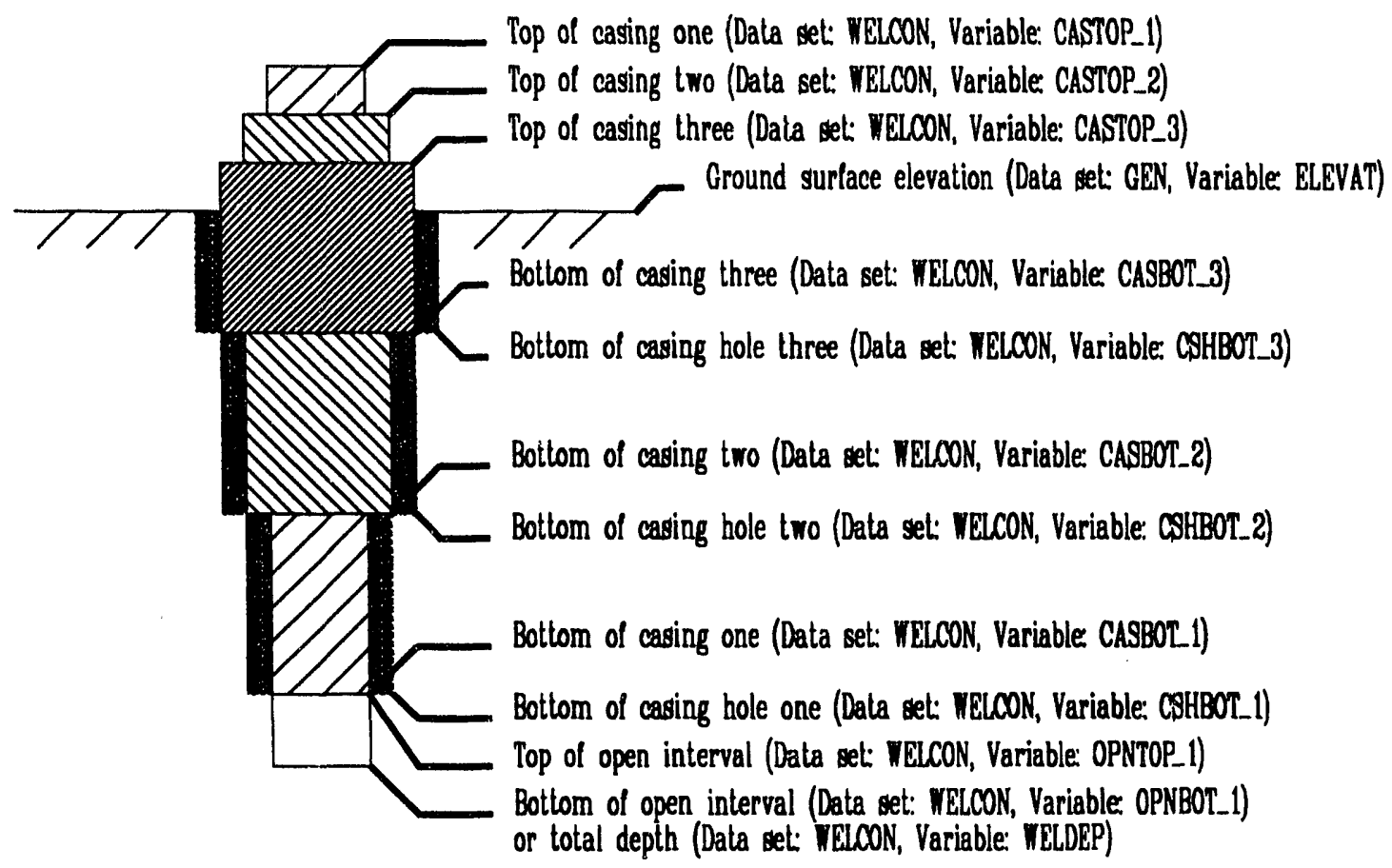

Key:

Grout

DZA Casing one

MIII Casing tro

UTIIIIA Casing three

63. Diagram of an open well with three casings. 


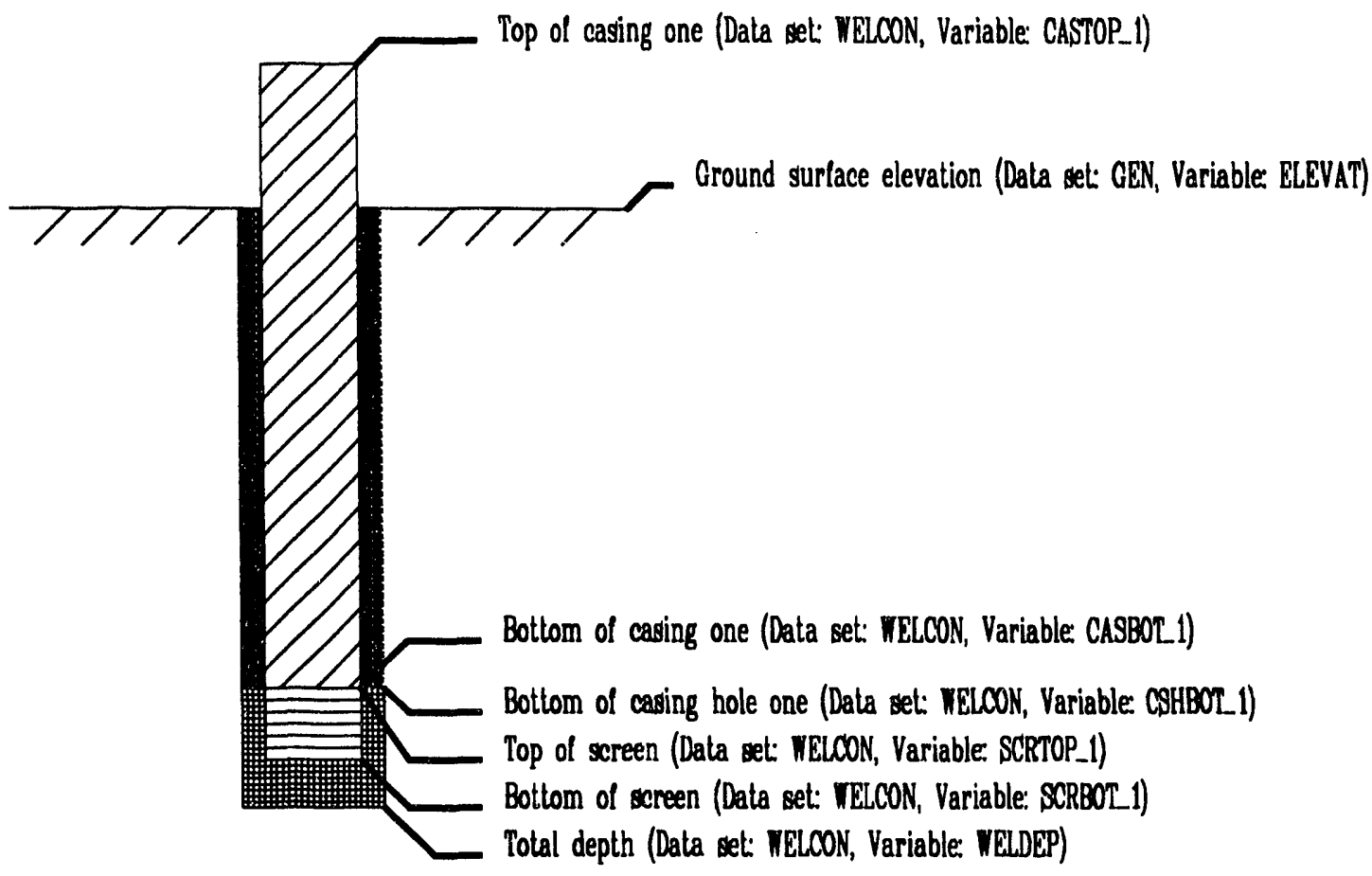

Key.

Grout

Pack material

VZA Casing one

6.4. Diagram of a screened well with one casing. 


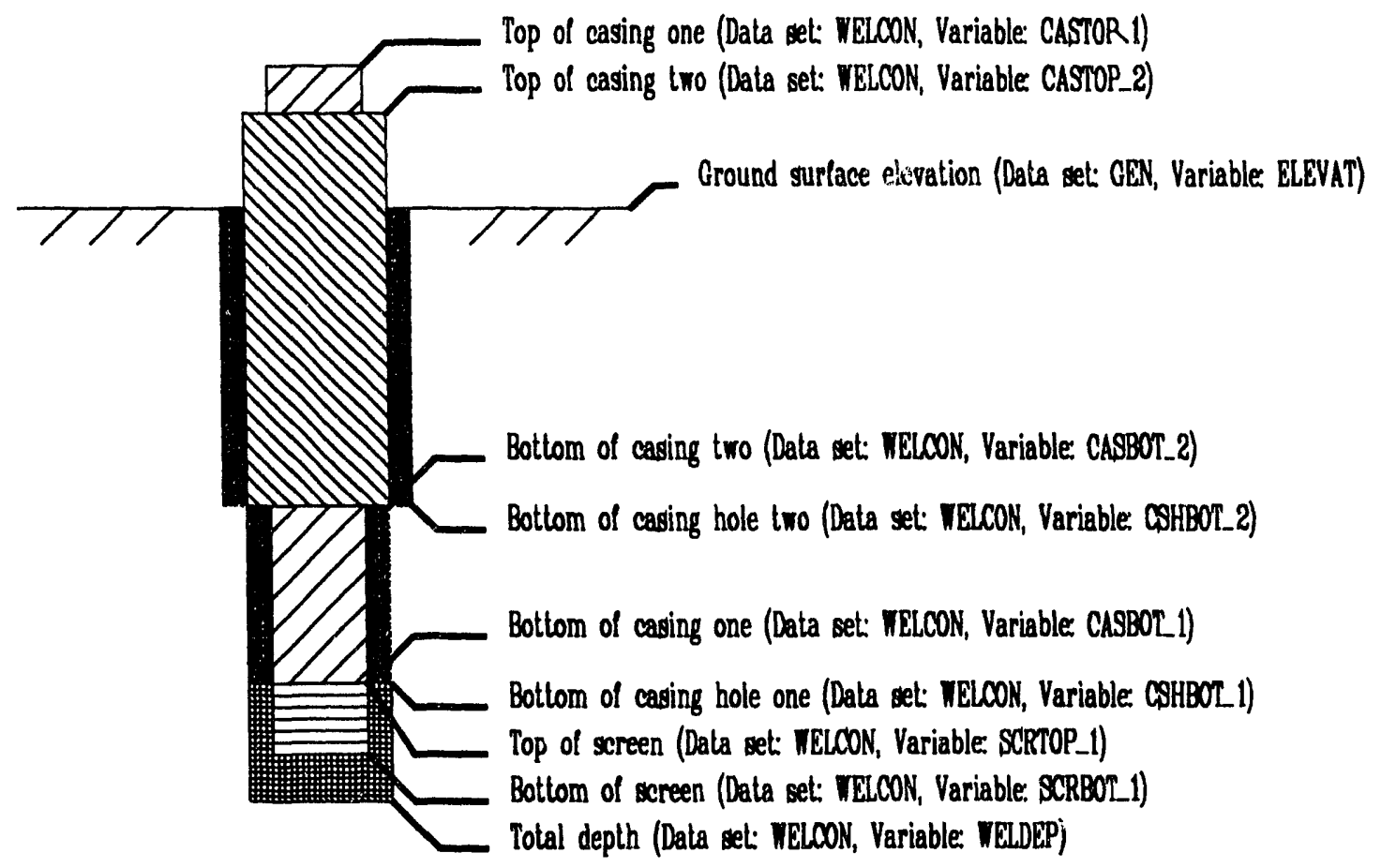

Ker.

Crout
Pack material
Casing one
120

65. Diagram of a screened well with two casings. 


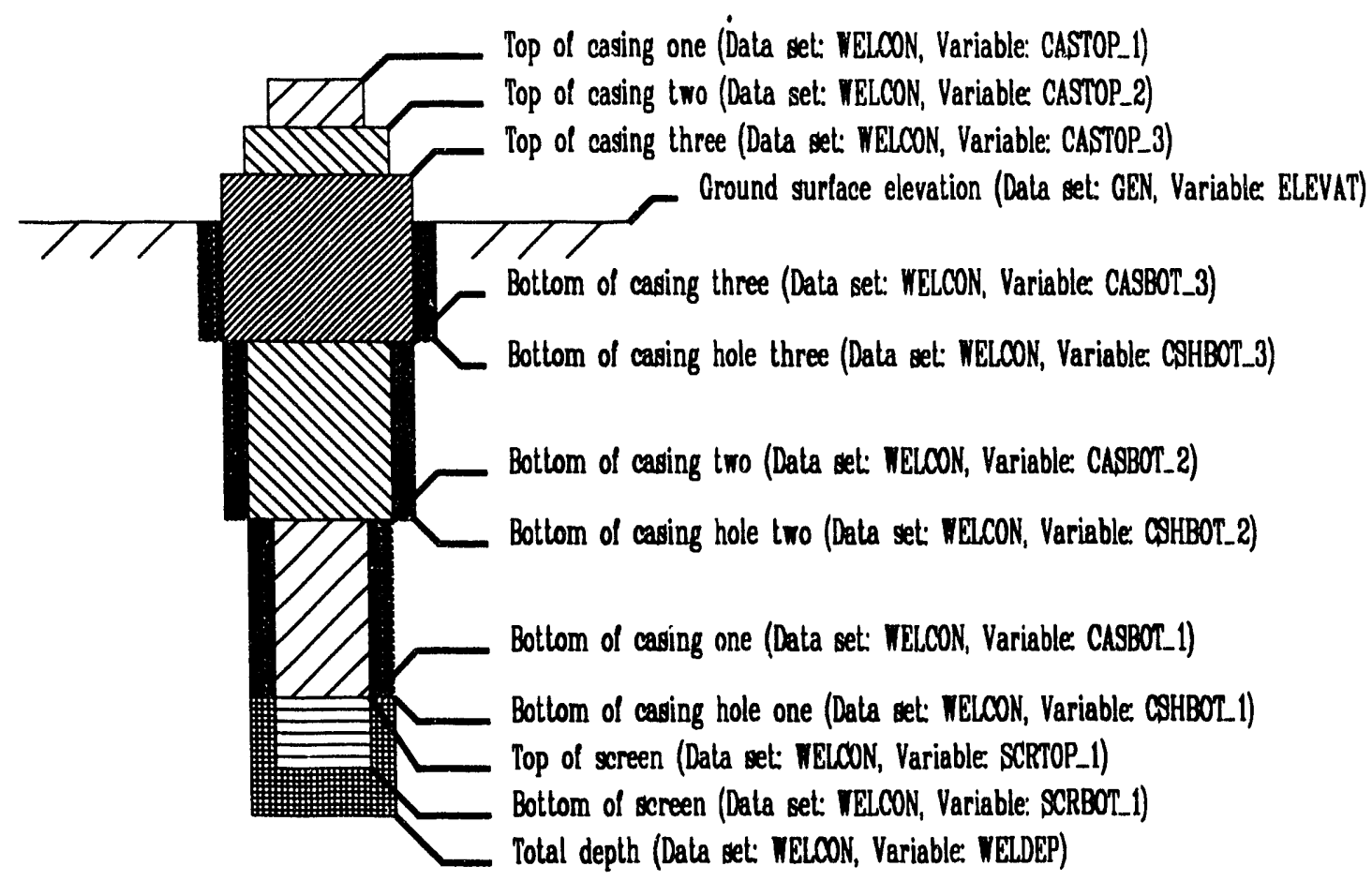

Key:

Grout

Pack material

Casing one

Casing tro

Casing three

66. Diagram of a screened well with three casings. 
7. CROSS-REFERENCE OF PARAMETERS, VARIABLES, AND DATA BASE FILES 
Table 7.1. Cross-reference of parameters, variables, and data base files

Parameters

Acenaphthene

Acenaphthylene

Acetone

Acrolein

Acrylonitrile

Active or inactive well

Aldrin

Alkalinity, bicarbonate (as $\mathrm{CaCO}_{3}$ )

Alkalinity, carbonate (as $\mathrm{CaCO}_{3}$ )

Alkalinity, total (as $\left.\mathrm{CaCO}_{3}\right)$

Alternate well name

Aluminum (ICAP)

Americium-241

Ammonia

Aniline

Anthracene

Antimony (ICAP)

Antimony-125

Aquifer

Arsenic (AAS)

Arsenic (ICAP)

Barium (AAS)

Barium (ICAP)

Benzene

Benzidine

Benzo[a]anthracene

Benzo $[b]$ fluoranthene

Benzo[k]fluoranthene

Benzo[ghi]perylene

Benzo[a]pyrene

Benzoic acid

Benzyl alcohol

Beryllium (AAS)

Beryllium (ICAP)

$\alpha$-BHC

$\beta$-BHC

$\delta$-BHC

$\gamma$-BHC

Biochemical oxygen demand

Bis(2-chloroethyl)ether

Bis(2-chloroethoxy)methane
Variables

Data Base file

$\begin{array}{ll}\text { ACNTHE } & \text { BASE } \\ \text { ACNTHY } & \text { BASE } \\ \text { ACETON } & \text { VOC } \\ \text { ACROLN } & \text { VOC } \\ \text { ACRYLT } & \text { VOC } \\ \text { ACT_INAC } & \text { GEN } \\ \text { ALDRIN } & \text { PEST } \\ \text { ALKBCR } & \text { ANION } \\ \text { ALKCAR } & \text { ANION } \\ \text { ALKTL } & \text { ANION } \\ \text { WELALT_1 } & \text { GEN } \\ \text { ALUM_I } & \text { METAL } \\ \text { AM_241 } & \text { RAD } \\ \text { AMMONI } & \text { MISC } \\ \text { ANILIN } & \text { BASE } \\ \text { ANTHRA } & \text { BASE } \\ \text { ANT_I } & \text { METAL } \\ \text { ANT125 } & \text { RAD } \\ \text { AQUIFER } & \text { GEN } \\ \text { ARSN_A } & \text { METAL } \\ \text { ARSN_I } & \text { METAL } \\ \text { BARI_A } & \text { METAL } \\ \text { BARIII } & \text { METAL } \\ \text { BENZEN } & \text { VOC } \\ \text { BEN } & \text { BASE } \\ \text { BENAAN } & \text { BASE } \\ \text { BENBFL } & \text { BASE } \\ \text { BENKFL } & \text { BASE } \\ \text { BENPER } & \text { BASE } \\ \text { BENPYR } & \text { BASE } \\ \text { BENACD } & \text { ACID } \\ \text { BENZAI } & \text { BASE } \\ \text { BERY_A } & \text { METAL } \\ \text { BERY_I } & \text { METAL } \\ \text { BHC_A } & \text { PEST } \\ \text { BHC_B } & \text { PEST } \\ \text { BHC_D } & \text { PEST } \\ \text { BHC_G } & \text { PEST } \\ \text { BIOXD } & \text { MISC } \\ \text { BISETH } & \text { BASE } \\ \text { BISMTH } & \text { BASE } \\ & \\ & \end{array}$


Table 7.1 (continued)

Parametc.s

Variables

Data Base file

Bis(2-chloroisopropyl)ether

BISETP

BASE

Bis(2-ethylhexyl)phthalate

Boron (ICAP)

Bromide

Bromodichloromethane

Bromoform

Bromomethane

4-Bromophenyl phenyl ether

2-Butanone

1-Butene

Butyl benzyl phthalate

Cadmium (AAS)

Cadmium (ICAP)

Cadmium (Polar)

Calcium (ICAP)

Carbon disulfide

Carbon tetrachloride

Casing one bottom depth

Casing two bottom depth

Casing three bottom depth

Casing one diameter

Casing two diameter

Casing one inside diameter

Casing one outside diameter

Casing two outside diameter

Casing three outside diameter

Casing one hole depth

Casing two hole depth

Casing one hole diameter

Csing two hole diameter

Casing three hole diameter

Casing one material

Casing two material

Casing three material

Casing one top elevation

Cerium-144

Cesium-134

Cesium-137

Chemical oxygen demand

Chemical data list

\begin{tabular}{|c|c|}
\hline BISPHT & BASE \\
\hline BOR_I & METAL \\
\hline BROM & ANION \\
\hline BROMID & VOC \\
\hline BROMOF & VOC \\
\hline BROMOM & VOC \\
\hline BROPHE & BASE \\
\hline BUTANO & VOC \\
\hline BUTENE & VOC \\
\hline BUTBPH & BASE \\
\hline CADM_A & METAL \\
\hline CADM_I & METAL \\
\hline CADM_P & METAL \\
\hline CALC_I & METAL \\
\hline CARDĪS & VOC \\
\hline CARTET & VOC \\
\hline CASBOT_1 & WCON \\
\hline CASBOT_2 & WCON \\
\hline CASBOT_3 & WCON \\
\hline CASDI_1 & WCON \\
\hline CASDI_2 & WCON \\
\hline CASDĪ_1 1 & WCON \\
\hline CASDIO_1 & WCON \\
\hline CASDIO_2 & WCON \\
\hline CASDIO_3 & WCON \\
\hline CSHBOT_1 & WCON \\
\hline CSHBOT_2 & WCON \\
\hline CSHDI_1 & WCON \\
\hline CSHDI_ 2 & WCON \\
\hline CSHDI_3 & WCON \\
\hline CASMĀT_1 & WCON \\
\hline CASMAT_2 & WCON \\
\hline CASMAT_3 & WCON \\
\hline CASTOP_1 & WCON \\
\hline CER144 & RAD \\
\hline CES134 & RAD \\
\hline CES137 & RAD \\
\hline CHMOXD & MISC \\
\hline CHMDAT & GEN \\
\hline
\end{tabular}


Table 7.1 (continued)

Parameters

Variables

Data Base file

Chlordane

$\alpha$-Chlordane

$\boldsymbol{\gamma}$-Chlordane

Chloride

4-Chloroaniline

Chlorobenzene

Chlorodibromomethane

Chloroethane

2-Chloroethyl vinyl ether

Chloroform

Chloromethane

4-Chloro-3-methylphenol

2-Chloronaphthalene

2-Chlorophenol

4-Chlorophenyl phenyl ether

Chlorotrifluoroethene

Chromium (AAS)

Chromium (ICAP)

Chromium (Polar)

Chrysene

Cobalt (ICAP)

Cobalt-60

Coliform

Conductivity, laboratory, first measurement

Conductivity, laboratory, second measurement

Conductivity, laboratory, third measurement

Conductivity, laboratory, fourth measurement

Conductivity at development

Conductivity, field, first measurement

Conductivity, field, second measurement

Conductivity, field, third measurement

Conductivity, field, fourth measurement

Conductivity, field, fifth measurement

Conductivity, field, sixth measurement

Conductivity, field, seventh measurement

Construction comment one

Construction comment two

Construction comment three

Construction comment four

Construction comment five

Construction comment six

$\begin{array}{ll}\text { CLRD } & \text { PEST } \\ \text { CLRD_A } & \text { PEST } \\ \text { CLRD_G } & \text { PEST } \\ \text { CHLOR } & \text { ANION } \\ \text { CHLANI } & \text { BASE } \\ \text { CHLBEN } & \text { VOC } \\ \text { CHLDIB } & \text { VOC } \\ \text { CHLETH } & \text { VOC } \\ \text { CHLEVE } & \text { VOC } \\ \text { CHLFOR } & \text { VOC } \\ \text { CHLMET } & \text { VOC } \\ \text { CLMETH } & \text { ACID } \\ \text { CHLNAP } & \text { BASE } \\ \text { CLPHEN } & \text { ACID } \\ \text { CHLPPE } & \text { BASE } \\ \text { CHLTRI } & \text { VOC } \\ \text { CHRO_A } & \text { METAL } \\ \text { CHRO_I } & \text { METAL } \\ \text { CHRO_P } & \text { METAL } \\ \text { CHRYSE } & \text { BASE } \\ \text { COBL_I } & \text { METAL } \\ \text { COBL60 } & \text { RAD } \\ \text { COLIFO } & \text { MISC } \\ \text { CON_L1 } & \text { MISC } \\ \text { CON_L2 } & \text { MISC } \\ \text { CON_L3 } & \text { MISC } \\ \text { CON_LA } & \text { MISC } \\ \text { COND_DEV } & \text { WCON } \\ \text { CON_F1 } & \text { FIELD } \\ \text { CON_F2 } & \text { FIELD } \\ \text { CON_F3 } & \text { FIELD } \\ \text { CON_F4 } & \text { FIELD } \\ \text { CON_F5 } & \text { FIELD } \\ \text { CON_F6 } & \text { FIELD } \\ \text { CON_F7 } & \text { FIELD } \\ \text { COMCON_1 } & \text { WCON } \\ \text { COMCON_2 } & \text { WCON } \\ \text { COMCON_3 } & \text { WCON } \\ \text { COMCON_4 } & \text { WCON } \\ \text { COMCON_5 } & \text { WCON } \\ \text { COMCON_6 } & \text { WCON }\end{array}$


Table 7.1 (continued)

\begin{tabular}{|c|c|c|}
\hline Parameters & Variables & Data Base file \\
\hline Construction comment seven & $\mathrm{COMCON}_{-} 7$ & WCON \\
\hline Construction comment eight & COMCON_8 & WCON \\
\hline Construction date & DATE_CON & WCON \\
\hline Copper (ICAP) & COPP I & METAL \\
\hline Cyanide & CYANID & ANION \\
\hline Cyclohexane & CYCHEX & VOC \\
\hline $2,4-D$ & D_24 & BASE \\
\hline 4,4'-DDD & DD̄D_44 & PEST \\
\hline 4,4'-DDE & $\mathrm{DDE}_{-44}^{-}$ & PEST \\
\hline 4,4'-DDT & $\mathrm{DDT}_{-}^{-44}$ & PEST \\
\hline Destruction date & DATE_DES & WCON \\
\hline Diacetone alcohol & DIACĀL & BASE \\
\hline Dibenzo $[a, h]$ anthracene & DIBANT & BASE \\
\hline Dibenzofuran & DIBFUR & BASE \\
\hline 1,2-Dichlorobenzene & DIC_12 & BASE \\
\hline 1,3-Dichlorobenzene & DIC_13 & BASE \\
\hline 1,4-Dichlorobenzene & DIC_14 & BASE \\
\hline 3,3'-Dichlorobenzidine & $\mathrm{DIC}_{3} 33$ & BASE \\
\hline Dichlorodifluoromethane & DCD̄IF & VOC \\
\hline 1,1-Dichloroethane & DCEA11 & VOC \\
\hline 1,2-Dichloroethane & DCEA12 & VOC \\
\hline 1,1-Dichloroethene & DCEE11 & VOC \\
\hline 1,2-Dichloroethene & DCEE12 & VOC \\
\hline trans-1,2-Dichloroethene & DCET12 & VOC \\
\hline 2,4-Dichlorophenol & DCP_24 & ACID \\
\hline 1,2-Dichloropropane & DCP12 & VOC \\
\hline cis-1,3-Dichloropropene & DCPC13 & VOC \\
\hline trans-1,3-Dichloropropene & DCPT13 & VOC \\
\hline 1,2-Dichloro-1,1,2,2-tetrafluoroethane & DCTET & VOC \\
\hline 1,2-Dichloro-1,1,2-trifluoroethane & DCTRI & VOC \\
\hline Dieldrin & DIELDR & PEST \\
\hline Diethyl phthalate & DIEPHT & BASE \\
\hline 2,3-Dimethylbutane & DMBT23 & VOC \\
\hline 2,4-Dimethylpentane & DMPN24 & VOC \\
\hline 2,4-Dimethylphenol & DMP_24 & ACID \\
\hline Dimethyl phthalate & DMTPHT & BASE \\
\hline Di-n-butyl phthalate & DNBPHT & BASE \\
\hline 4,6-Dinitro-2-methylphenol & DNM_46 & ACID \\
\hline 2,4-Dinitrophenol & DNP_24 & ACID \\
\hline 2,4-Dinitrotoluene & DNI_24 & BASE \\
\hline
\end{tabular}


Table 7.1 (continued)

2,6-Dinitrotoluene

Di-n-octyl phthalate

Dissolved oxygen, first measurement

Dissolved oxygen, second measurement

Dissolved oxygen, third measurement

Dissolved oxygen, fourth measurement

East coordinate (plant)

Elevation

$\alpha$-Endosulfan

$\beta$-Endosulfan

Endosulfan sulfate

Endrin

Endrin aldehyde

Endrin ketone

Ethylbenzene

Ethylcyclobutane

1-Ethyl-2-methyl-benzene

Fecal coliform

Fluoranthene

Fluorene

Fluoride

Fluorotrichloromethane

Fresh rock top

Gallium (ICAP)

Geological formation

Grid

Gross alpha activity

Gross beta activity

Gross gamma activity

Hardness

Heptachlor

Heptachlor epoxide

Hexachlorobenzene

Hexachlorobutadiene

Hexachlorocyclopentadiene

Hexachloroethane

Hexamethylcyclotrisiloxane

2-Hexanone

Hydraulic conductivity

Hydroxyl

\begin{tabular}{|c|c|}
\hline DNI_26 & BASE \\
\hline DNOCTP & BASE \\
\hline DOXY_1 & FIELD \\
\hline DOXY_2 & FIELD \\
\hline DOXY_3 & FIELD \\
\hline DOXY_4 & FIELD \\
\hline EAST & GEN \\
\hline ELEVAT & GEN \\
\hline ENSU A & PEST \\
\hline ENSU_B & PEST \\
\hline ENSULS & PEST \\
\hline END & PEST \\
\hline ENDALD & PEST \\
\hline ENDKET & PEST \\
\hline ETBENZ & VOC \\
\hline ETCYCB & VOC \\
\hline ETMETB & VOC \\
\hline FECCOL & MISC \\
\hline FLUORA & BASE \\
\hline FLUORE & BASE \\
\hline FLUOR & ANION \\
\hline FLTRIC & VOC \\
\hline ROCTOPFR & GEN \\
\hline GALIII · & METAL \\
\hline GEO_FORM: & GEN \\
\hline GRID & GEN \\
\hline$A C T$ A & RAD \\
\hline ACT_B & RAD \\
\hline ACT_G & RAD \\
\hline HARD & MISC \\
\hline HEPTCH & PEST \\
\hline HEPTEP & PEST \\
\hline HXCBEN & BASE \\
\hline HXCBUT & BASE \\
\hline HXCCYC & BASE \\
\hline HXCETA & BASE \\
\hline HEXAMT & VOC \\
\hline HEXANO & VOC \\
\hline HYDCON & GEN \\
\hline HYDROX & MISC \\
\hline
\end{tabular}


Table 7.1 (continued)

\begin{tabular}{|c|c|c|}
\hline Parameters & Variables & Data Base \\
\hline Indeno[1,2,3-cd]pyrene & INDPYR & BASE \\
\hline Iodide & IODIDE & ANION \\
\hline Iodine -125 and iodine -129 & I_1259 & RAD \\
\hline Iodine-131 & I_131 & RAD \\
\hline Iron (AAS) & $\overline{I R O N} \_A$ & METAL \\
\hline Iron (ICAP) & IRON_I & METAL \\
\hline Isophorone & ISOPR̄O & BASE \\
\hline Lead (AAS) & LEAD_A & METAL \\
\hline Lead (Polar) & LEAD_P & METAL \\
\hline Lithium (ICAP) & LTTH_I & METAL \\
\hline Logs, existence of & LOGS̄_Q & GEN \\
\hline Magnesium (ICAP) & MAGN_I & METAL \\
\hline Manganese (AAS) & MANG_A & METAL \\
\hline Manganese (ICAP) & MANG_I & METAL \\
\hline Measure point & MP & WCON \\
\hline Measure point correction & MP_COR & WCON \\
\hline Mercury (CVAA) & MER̄C_V & METAL \\
\hline Methoxychlor & METHŌX & PEST \\
\hline 2-Methoxy-2-methylpropane & METOX & VOC \\
\hline 2-Methyl-butane & MTHBUT & VOC \\
\hline Methylcyclohexane & MTHCYH & VOC \\
\hline Methylcyclopentane & MTHCYP & VOC \\
\hline Methylene chloride & MTHECL & VOC \\
\hline 2-Methylnaphthalene & MTNP_2 & BASE \\
\hline 2-Methylpentane & MTHP_2 & VOC \\
\hline 3-Methylpentane & MTHP_3 & VOC \\
\hline 4-Methyl-2-pentanone & MTHPẼN & VOC \\
\hline 2-Methylphenol & METH_2 & ACID \\
\hline 4-Methylphenol & METH_4 & ACID \\
\hline 2-Methyl-1-propane & MTHPR̄O & VOC \\
\hline Molybdenum (ICAP) & MOLY_I & METAL \\
\hline Naphthalene & NAPHT̄A & BASE \\
\hline$N$-butyl benzenesulfonamide & NBUTBZ & BASE \\
\hline Neptunium-237 & NEP237 & RAD \\
\hline$N$-Nitrosodimethylamine & NTMETH & BASE \\
\hline$N$-Nitrosodi- $n$-propylamine & NTNPRO & BASE \\
\hline$N$-Nitrosodiphenylamine & NTPHEN & BASE \\
\hline Nickel (ICAP) & NICK_I & METAL \\
\hline Niobium (ICÁP) & NIOB_I & METAL \\
\hline Niobium-95 & NIOB $\overline{9} 5$ & RAD \\
\hline Nitrate (as $\mathbf{N}$ ) & NITRAT & ANION \\
\hline
\end{tabular}


Table 7.1 (continued)

Parameters

2-Nitroaniline

Variables

Data Base file

3-Nitroaniline

NITRIT

NTAN_2

NTAN_3

4-Nitroaniline

NTAN 4

Nitrobenzene

Nitrogen (Kjeldahl)

NTBEN̄Z

2-Nitrophenol

4-Nitrophenol

North coordinate

Open interval one bottom

Open interval two bottom

Open interval one diameter

Open interval two diameter

NIT_KJ

NITP_2

NITP_4

NORTH

OPNBOT_1

OPNBOT_2

OPNDI 1

OPNDI-2

Open interval one top

Open interval two top

OPNTOP_1

OPNTOP 2

Open or screened well

OPEN_SCR

Pack interval bottom

PACKB̄OT

Pack interval top

PCB (Aroclor 1016)

PACKTOP

PCB (Aroclor 1221)

PCB_16

PCB_21

$\mathrm{PCB}_{-}^{-32}$

PCB (Aroclor 1232)

PCB (Aroclor 1242)

PCB (Aroclor 1248)

PCB (Aroclor 1254)

PCB (Aroclor 1260)

Pentachlorophenol

$\mathrm{pH}$, field, first measurement

$\mathrm{pH}$, field, second measurement

$\mathrm{pH}$, field, third measurement

$\mathrm{pH}$, field, fourth measurement

$\mathrm{PCB}_{-} 42$

$\mathrm{PCB}_{-48}^{-42}$

$\mathrm{PCB}_{-54} 5$

PCB_60

PENTCL

PH_F1

PH_F2

PH_F3

PH_F4

PH_F5

PH_F6

PH_F7

PH_L1

PH_L2

$\mathrm{PH}_{-} \mathrm{L} 3$

PH_L4

PHĒNAN

PHENOL

PHN_TL

Phenols (total)

Phosphate

PHOS̄PA

ANION
BASE
BASE
BASE
BASE
MISC
ACID
ACID
GEN
WCON
WCON
WCON
WCON
WCON
WCON
GEN
WCON
WCON
PCB
PCB
PCB
PCB
PCB
PCB
PCB
ACID
FIELD
FIELD
FIELD
FIELD
FIELD
FIELD
FIELD
MISC
MISC
MISC
MISC
BASE
ACID
MISC
ANION


Table 7.1 (continued)

Phosphorus (ICAP)

Plutonium-238

Plutonium-239

Plutonium-239 and plutonium-240

Potassium (Flame - emission)

Potassium (ICAP)

Protactinium

Pyrene

Radium

Radium-226

Radium-228

Redox

Rock top

Ruthenium-106

Screen one bottom

Screen two bottom

Screen three bottom

Screen four bottom

Screen one diameter

Screen one inside diameter

Screen one material

Screen slot size

Screen one top

Screen two top

Screen three top

Screen four top

Seal bottom

Seal top

Selenium (AAS)

Selenium (ICAP)

Silicon (ICAP)

Silver (AAS)

Silver (ICAP)

Silvex

Site

Sodium (AAS)

Sodium (ICAP)

Source of acid data

Source of anion data

Source of base/neutral data

Source of field data

$\begin{array}{ll}\text { PHOS_I } & \text { METAL } \\ \text { P_238 } & \text { RAD } \\ \text { P_239 } & \text { RAD } \\ \text { P_2394 } & \text { RAD } \\ \text { POTA_E } & \text { METAL } \\ \text { POTA_I } & \text { METAL } \\ \text { PROTAC } & \text { RAD } \\ \text { PYRENE } & \text { BASE } \\ \text { RAD } & \text { RAD } \\ \text { RAD226 } & \text { RAD } \\ \text { RAD228 } & \text { RAD } \\ \text { REDOX } & \text { FIELD } \\ \text { ROCTOP } & \text { GEN } \\ \text { RUT106 } & \text { RAD } \\ \text { SCRBOT_1 } & \text { WCON } \\ \text { SCRBOT_2 } & \text { WCON } \\ \text { SCRBOT_3 } & \text { WCON } \\ \text { SCRBOT_4 } & \text { WCON } \\ \text { SCRDI_1 } & \text { WCON } \\ \text { SCRDII_1 } & \text { WCON } \\ \text { SCRMAT_1 } & \text { WCON } \\ \text { SCRSLTSZ } & \text { WCON } \\ \text { SCRTOP_1 } & \text { WCON } \\ \text { SCRTOP_2 } & \text { WCON } \\ \text { SCRTOP_3 } & \text { WCON } \\ \text { SCRTOP_4 } & \text { WCON } \\ \text { SEALBOT } & \text { WCON } \\ \text { SEALTOP } & \text { WCON } \\ \text { SELE_A } & \text { METAL } \\ \text { SELE_I } & \text { METAL } \\ \text { SILIII } & \text { METAL } \\ \text { SILV_A } & \text { METAI } \\ \text { SILVII } & \text { METAL } \\ \text { SILVEX } & \text { PEST } \\ \text { SITE } & \text { GEN } \\ \text { SODI_A } & \text { METAL } \\ \text { SODI_I } & \text { METAL } \\ \text { SRC_ACID } & \text { ACID } \\ \text { SRC_AN } & \text { ANION } \\ \text { SRC_BASE } & \text { BASE } \\ \text { SRC_FLD } & \\ & \end{array}$


Table 7.1 (continued)

Parameters

Variables

Data Base file

Source of general data

Source of metal data

Source of miscellaneous data

Source of PCB data

Source of pesticide data

Source of radionuclide data

Source of volatile organic compound data

Source of well construction data

Strontium

Strontium (ICAP)

Strontium-90

Styrene

Sulfate

Sulfide

2,4,5-T

Technetium-99

Temperature, field, first measurement

Temperature, field, second measurement

Temperature, field, third measurement

Temperature, field, fourth measurement

Temperature, field, fifth measurement

Temperature, field, sixth measurement

Temperature, field, seventh measurement

1,1,2,2-Tetrachloroethane

Tetrachloroethene

Tetramethylsilane

Thallium (AAS)

Thallium-228

Thorium (ICAP)

Thorium-228

Thorium-230

Thorium-232

Thorium-234

Thorium-231 and thorium-234

Tin (AAS)

Tin (ICAP)

Titanium (ICAP)

Toluene

Total dissolved solids

Total organic carbon, first measurement

Total organic carbon, second measurement

\begin{tabular}{|c|c|}
\hline SRC_GEN & GEN \\
\hline SRC_MET & METAL \\
\hline SRC-MISC & MISC \\
\hline SRC_PCB & PCB \\
\hline SRC_PEST & PEST \\
\hline SRC_RAD & RAD \\
\hline SRC_VOC & VOC \\
\hline SRC_WCON & WCON \\
\hline STRŌNT & RAD \\
\hline STRO_I & METAL \\
\hline STRN9̄0 & $\mathbf{R A D}$ \\
\hline STYREN & VOC \\
\hline SULFAT & ANION \\
\hline SULFID & ANION \\
\hline$T \_245$ & BASE \\
\hline TËC 99 & $\mathbf{R A D}$ \\
\hline TMP F1 & FIELD \\
\hline TMP F2 & FIELD \\
\hline TMP_F3 & FIELD \\
\hline TMP_F4 & FIELD \\
\hline TMP F5 & FIELD \\
\hline TMP_F6 & FIELD \\
\hline TMP F7 & FIELD \\
\hline TECETA & VOC \\
\hline TECETE & VOC \\
\hline TETMTS & VOC \\
\hline THAL A & METAL \\
\hline THA 28 & RAD \\
\hline THOR I & METAL \\
\hline THO_28 & RAD \\
\hline THO_30 & RAD \\
\hline THO 32 & RAD \\
\hline THO 34 & RAD \\
\hline THO314 & RAD \\
\hline $\operatorname{TIN} A$ & METAL \\
\hline TIN I & METAL \\
\hline $\operatorname{TITA} \mathbf{A}$ & METAL \\
\hline TOLŨEN & VOC \\
\hline TL DIS & MISC \\
\hline TOC 1 & MISC \\
\hline $\mathrm{TOC}^{-2}$ & MISC \\
\hline
\end{tabular}


Table 7.1 (continued)

Parameters

Variables

Data Base file

Total organic carbon, third measurement Total organic carbon, fourth measurement Total organic chloride, first measurement Total organic chloride, second measurement Total organic chloride, third measurement Total organic chloride, fourth measurement Total organic halides, first measurement Total organic halides, second measurement Total organic halides, third measurement

Total organic halides, fourth measurement

Total suspended solids

Toxaphene

Transmissivity

1,2,4-Trichlorobenzene

1,1,1-Trichloroethane

1,1,2-Trichloroethane

Trichloroethene

2,4,5-Trichlorophenol

2,4,6-Trichlorophenol

1,1,2-Trichloro-1,2,2-trifluoroethane

Trifluoroethene

1,3,5-Trimethylbenzene

Tritium

Turbidity

Uranium (Fluor)

Uranium (ICAP)

Uranium-234

Uranium-235 and uranium-236

Uranium-238

Vanadium (ICAP)

Vinyl acetate

Vinyl chloride

Waste Area Grouping

Water level

Weathered rock top

Well construction method

Well contractor

Well depth, construction

Well type one

Well type two

Xylenes, total

\begin{tabular}{|c|c|}
\hline TOC_3 & MISC \\
\hline $\mathrm{TOC}_{-4}$ & MISC \\
\hline TOCL_1 & MISC \\
\hline TOCL_2 & MISC \\
\hline $\mathrm{TOCL}_{-} 3$ & MISC \\
\hline TOCL_4 & MISC \\
\hline TOX_1 & MISC \\
\hline $\operatorname{TOX} 2$ & MISC \\
\hline $\operatorname{TOX}_{-3}^{-3}$ & MISC \\
\hline TOX_4 & MISC \\
\hline TL SÜS & MISC \\
\hline TOXAPH & PEST \\
\hline TRANS & GEN \\
\hline TB_124 & BASE \\
\hline TRC̄111 & VOC \\
\hline TRC112 & VOC \\
\hline TRICLE & VOC \\
\hline TC_245 & ACID \\
\hline TC-246 & ACID \\
\hline TRĪFLE & VOC \\
\hline TRICTR & VOC \\
\hline TRM135 & VOC \\
\hline TRIT & RAD \\
\hline TURBID & MISC \\
\hline URAN F & METAL \\
\hline URAN II & METAL \\
\hline U_234 & RAD \\
\hline$U^{-} 2356$ & RAD \\
\hline Ū238 & RAD \\
\hline VĀNA_I & METAI \\
\hline VNYAC̄E & VOC \\
\hline VNYCHL & VOC \\
\hline WAG & GEN \\
\hline WAT_LEV & MISC \\
\hline ROCT̄OPWT & GEN \\
\hline CONSMTHD & WCON \\
\hline CONTNAME & WCON \\
\hline WELDEP & WCON \\
\hline WELTYP 1 & GEN \\
\hline WELTYP_2 & GEN \\
\hline $\mathrm{ENE}^{-}$ & VOC \\
\hline
\end{tabular}


Table 7.1 (continued)

\begin{tabular}{lll} 
Parameters & Variables & Data Base file \\
\hline Zinc (ICAP) & ZINC_I & $\begin{array}{l}\text { METAL } \\
\text { METAL }\end{array}$ \\
\hline
\end{tabular}




\section{REFERENCES}

EPA (U.S. Environmental Protection Agency). 1989. Risk assessment guidance for Superfund. Vol. 1. Human Health Evaluation Manual (Part A). EPA/540/1-89/002.

Geraghty and Miller, Inc. 1989. Hydrogeology of the Oak Ridge Gaseous Diffusion Plant. K/Sub/85-22224/12.

Thompson, B. K. 1993. Management Plan for the Oak Ridge Reservation Hydrology and Geology Study Groundwater Data Base. ORNL/TM-12048. Oak Ridge National Laboratory, Oak Ridge, Tennessee.

Hook, L. A., L. D. Voorhees, M. J. Gentry, M. A. Faulkner, J. A. Shaakir-Ali, K. A. Newman, R. A. McCord, L. F. Goins, and P. T. Owen. 1989. Data Base Management Activities for the Remedial Action Program at ORNL: Calendar Year 1989. ORNL/ER-16. Oak Ridge National Laboratory, Oak Ridge, Tennessee.

Hook, L. A., L. D. Voorhees, M. J. Gentry, M. A. Faulkner, K A. Newman, R. A. McCord, A. E. Rosen, J. L. Bledsoe, and P. T. Owen. 1988. Data Base Management Activities for the Remedial Action Program at ORNL: Calendar Year 1988. ORNL/TM-11147. Oak Ridge National Laboratory, Oak Ridge, Tennessee.

King H. L., C. S. Haase, and B. K. Harrington. 1991. Updated Subsurface Data Base for Bear Creek Valley, Chestnut Ridge, and Parts of Bethel Valley on the U.S. Department of Energy Oak Ridge Reservation. Y/TS-735. Oak Ridge Y-12 Plant, Oak Ridge, Tennessee. 


\section{DISTRIBUTION}

1. L. D. Bates

2. D. T. Bell

3. T. M. Bonine

4. J. B. Cannon

5. R. B. Clapp

6. J. H. Cushman

7. R. K Davis

8. M. F. P. DeLozier

9. R. B. Dreier

10. N. W. Durfee

11. T. O. Early

12. D. E. Fowler

13. C. W. Gehrs

14. C. D. Goins

15. P. J. Halsey

16. S. G. Hildebrand

17. D. D. Huff

18. P. Kanciruk

19. R. O. Kennard

20. B. L. Kimmel

21. A. J. Kuhaida, Jr.

22. S. L. Laman

23. R. S. Loffman

24-26. D. M. Matteo

27. B. C. McClelland

28. B. W. McMaster

29. J. B. Murphy

30-31. P. T. Owen
32. R. Poling

33. D. E. Reichle

34. C. T. Rightmire

35. G. E. Rymer

36. P. A. Schrandt

37. F. E. Sharples

38. L A Shevenell

39. D. S. Shriner

40. R. G. Stansfield

41. S. H. Stow

42. J. H. Swanks

43. D. W. Swindle

44. J. Switek

45-46. J. F. Tardiff

47-51. B. K. Thompson

52. J. R. Trabalka

53. D. D. Van Hoesen

54. R. I. Van Hook

55. R. K. White

56. M. A. Wood

57. P. S. Wood

58. T. F. Zondlo

59-60. Central Research Library

61-63. ESD Library

64-68. ER Document Management Center

69-70. ORNL Laboratory Records

71. ORNL Patent Section

72. Office of Assistant Manager for Energy Research and Development, DOE Oak Ridge Operations Office, P.O. Box 2001, Oak Ridge, Tennessee 37831-8600

73. G. W. Bodenstein, DOE Oak Ridge Operations Office, P.O. Box 2001, Oak Ridge, TN 37831

74. J. F. Franklin, Bloedel Professor of Ecosystem Analysis, College of Forest Resources, University of Washington, Anderson Hall (AR-10), Seattle, WA 98195

75. C. Gist, DOE Oak Ridge Operations Office, P.O. Box 2001, Oak Ridge, IN 37831

76. R. C. Harriss, Institute for the Study of Earth, Oceans, and Space, Science and Engineering Research Building, University of New Hampshire, Durham, NH 03824

77. G. M. Hornberger, Professor, Department of Environmental Sciences, University of Virginia, Charlottesville, VA 22903

78. G. Y. Jordy, Director, Office of Program Analysis, Office of Energy Research, ER-30, G-226, U.S. Department of Energy, Washington, DC 20545

79. J. R. Kannard, Program Manager, Bechtel National, Inc., P.O. Box 350, Oak Ridge Corporate Center, 151 Lafayette Drive, Oak Ridge, TN 37831-0350

80-81. R. L. Nace, Branch Chief, Nonenrichment Facilities, Oak Ridge Program Division, Office of Eastern Area Programs, Office of Environmental Restoration, EM-423, Trevion 2, U.S. Department of Energy, Washington, DC 20585 
82. R. H. Olsen, Professor, Microbiology and Immunology Department, University of Michigan, Medical Science II, \#5605, 1301 East Catherine Street, Ann Arbor, MI 48109-0620

83. A. Patrinos, Director, Environmental Sciences Division, Office of Health and Environmental Research, ER-74, U.S. Department of Energy, Washington, DC 20585

84-85. R. C. Sleeman, DOE Oak Ridge Operations Office, P.O. Box 2001, Oak Ridge, TN 37831-8541

86-87. J. T. Sweeney, DOE Oak Ridge Operations Office, P.O. Box 2001, Oak Ridge, TN $37831-8541$

88. D. W. Swindle, Radian Corporation, 120 South Jefferson Circle, Oak Ridge, TN 37830

89-90. H. M. Thron, Chief, Enrichment Facilities, Oak Ridge Program Division, Office of Eastern Area Programs, Office of Environmental Restoration, EM-423, Trevion 2, U.S. Department of Energy, Washington, DC 20585

91. F. J. Wobber, Environmental Sciences Division, Office of Health and Environmental Research, ER-74, U.S. Department of Energy, Washington, DC 20585

92-93. Office of Scientific and Technical Information, P.O. Box 62, Oak Ridge, TN 37831 

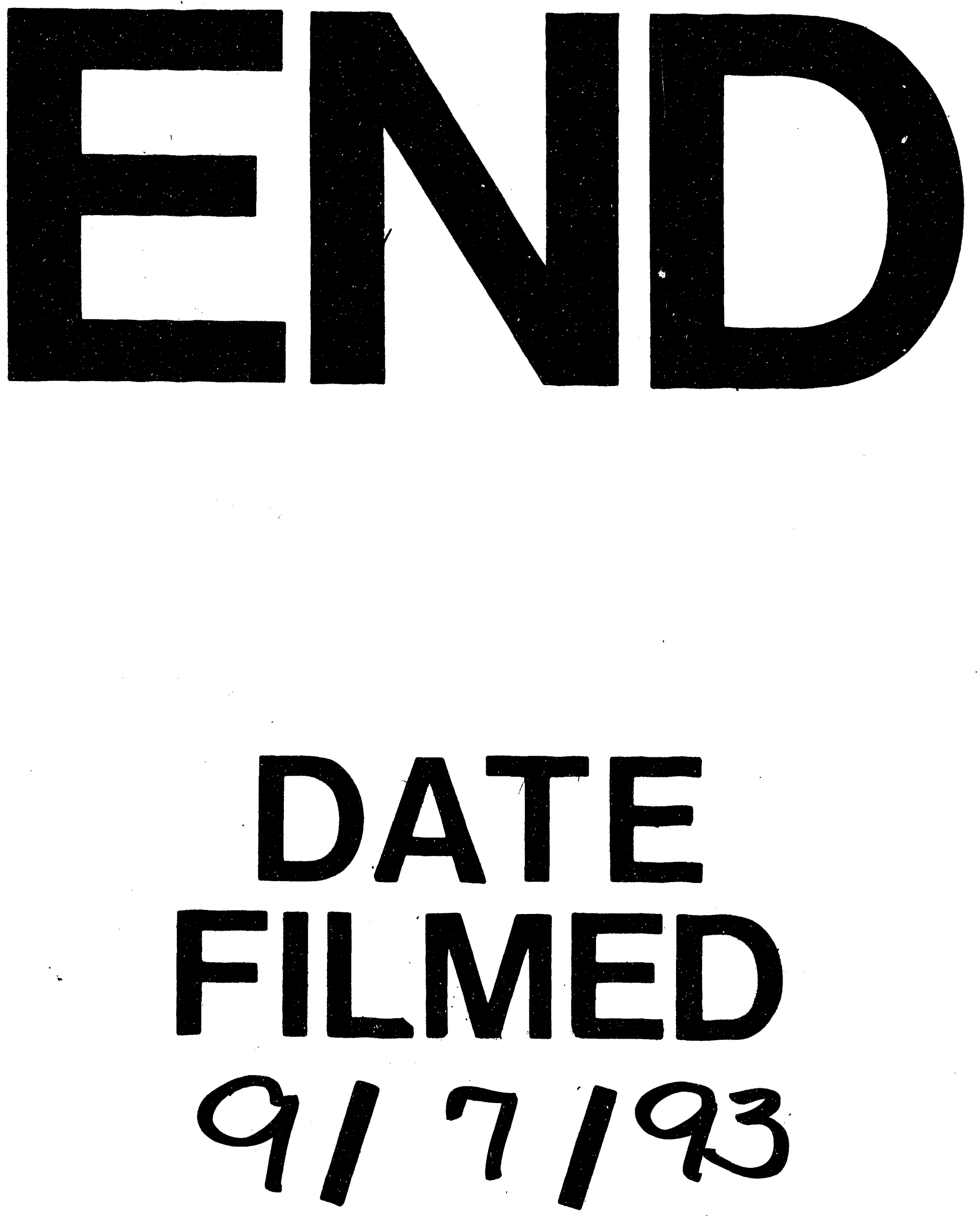

1 
